University of Louisville

ThinkIR: The University of Louisville's Institutional Repository

Electronic Theses and Dissertations

$12-2020$

\title{
Inclusion of classified staff in the implementation of positive behavior Interventions and supports: a district case study.
}

Devon M. Roberts

University of Louisville

Follow this and additional works at: https://ir.library.louisville.edu/etd

Part of the Teacher Education and Professional Development Commons

\section{Recommended Citation}

Roberts, Devon M., "Inclusion of classified staff in the implementation of positive behavior Interventions and supports: a district case study." (2020). Electronic Theses and Dissertations. Paper 3558.

https://doi.org/10.18297/etd/3558

This Doctoral Dissertation is brought to you for free and open access by ThinkIR: The University of Louisville's Institutional Repository. It has been accepted for inclusion in Electronic Theses and Dissertations by an authorized administrator of ThinkIR: The University of Louisville's Institutional Repository. This title appears here courtesy of the author, who has retained all other copyrights. For more information, please contact thinkir@louisville.edu. 
INCLUSION OF CLASSIFIED STAFF IN THE IMPLEMENTATION OF POSITIVE BEHAVIOR INTERVENTIONS AND SUPPORTS: A DISTRICT CASE STUDY

\author{
By \\ Devon M. Roberts \\ B. S., University of Louisville, 1995 \\ M.Ed., Indiana Wesleyan University, 1999 \\ Rank I, Western Kentucky University, 2001
}

\begin{abstract}
A Dissertation
Submitted to the Faculty of the

College of Education and Human Development of the University of Louisville in Partial Fulfillment of the Requirements

for the Degree of
\end{abstract}

Doctor of Education in Educational Leadership and Organizational Development

Department of Educational Leadership, Evaluation, and Organizational Development University of Louisville

Louisville, Kentucky

December 2020 
Copyright 2020 by Devon M. Roberts

All rights reserved 

INCLUSION OF CLASSIFIED STAFF IN THE IMPLEMENTATION OF POSITIVE BEHAVIOR SUPPORTS AND INTERVENTION: A DISTRICT CASE STUDY

\author{
By \\ Devon M. Roberts \\ B. S., University of Louisville, 1995 \\ M.Ed., Indiana Wesleyan University, 1999 \\ Rank I, Western Kentucky University, 2001
}

A Dissertation Approved on

October 6, 2020

By the following Dissertation Committee

Dr. William Kyle Ingle (Chair)

Dr. Harrie Buecker

Dr. Mary Brydon-Miller

Dr. Terry Scott 


\section{DEDICATION}

This dissertation is dedicated to my grandmother, Mrs. Margaret Wright. She has been an educator her entire life, and it is because of her that I went into the field of education. She has always been my teacher, mentor, advisor, and confidant. If I am but only a fraction of the woman she is, then I will be content. Grandma, this dissertation is for you. 


\section{ACKOWLEDGEMENTS}

It is with much gratitude that I acknowledge the work of my chair, Dr. Kyle Ingle. Your ability to help me realize my thoughts in a meaningful way has allowed me grow as a learner. You challenged me and pushed me to think bigger, and my work is better because of that support. I also want to thank Dr. Mary Brydon-Miller for your constant guidance and availability to talk, listen, and share ideas and progress in a safe space. Dr. Harrie Bucker and Dr. Terry Scott, you both provided insight during the process that was supportive and encouraging, and you made me feel like I was truly capable of doing this work. I also want to thank my first advisor in this program, Dr. Detra Johnson, who is always a cheerleader and advice giver.

I also want to thank my family who has always believed in me, supported me, and put up with all of my adventures. Every few years, I embark on a new journey and you all have constantly shown your support. I could not do anything without you all. I especially want to thank my mother, Nancy Lasley, my first teacher, my first friend, and my best friend. Your strength has always led me to be stronger and more driven than I ever planned. Because of you, I never give up. Because of you, I continue to try new things and strive to reach new goals. 


\section{ABSTRACT \\ INCLUSION OF CLASSIFIED STAFF IN THE IMPLEMENTATION OF POSITIVE BEHAVIOR SUPPORTS AND INTERVENTION: A DISTRICT CASE STUDY}

Devon M. Roberts

October 6, 2020

This qualitative case study examined the current use of Positive Behavior Interventions and Supports (PBIS) within a large school district and how that district includes classified staff within the implementation process. This study utilized information from interviews and focus groups of district leaders, school leaders, and classified staff to evaluate their perspectives on the daily use of PBIS, specifically how classified staff are included in all aspects of the initiative. Using the Normalization Process Theory (NPT) as a theoretical framework, this study sought to make sense of how complex organizational initiatives are intertwined and become embedded into the normal practices of everyday work within an organization. This case study focused on the voices of the staff whom are responsible for implementation of complex initiatives and provides perspectives that can inform policy and practice for other school districts that use PBIS and whom wish to ensure that all staff members have access to training and support that will lead to successful implementation district-wide. Through thick narrative descriptions of the work in action, this study provides evidence of the potential impact on practice that is timely and informative. 
Study findings revealed the need for explicit training for classified staff to ensure that all staff members have appropriate training and support to implement PBIS effectively. Additionally, study findings revealed that an emphasis on building relationships with students supports PBIS implementation and improves overall school climate and culture. The findings of this study provide recommendations at both the local level and in a broader context for other school districts that plan to use this program. 
TABLE OF CONTENTS

DEDICATION

ACKOWLEDGEMENTS

ABSTRACT $\quad$ V

LIST OF FIGURES $\quad \mathrm{X}$

LIST OF TABLES $\quad$ xi

CHAPTER I: INTRODUCTION 1

Purpose of the Study 4

Research Questions $\quad 4$

Significance of the Study $\quad 5$

$\begin{array}{ll}\text { Definition of Terms } & 7\end{array}$

$\begin{array}{lc}\text { Limitations and Delimitations of the Study } & 8\end{array}$

$\begin{array}{ll}\text { Organization of the Study } & 10\end{array}$

CHAPTER II: LITERATURE REVIEW

PBIS: A Brief Primer 13

Historical Context of PBIS $\quad 15$

$\begin{array}{ll}\text { PBIS Research } & 21\end{array}$

Equity and Social Justice Aims of PBIS 22

PBIS and Student Outcomes 26

Ensuring PBIS Success 30

Classified Staff Inclusion in School Initiatives 36

Normalization Process Theory $\quad 42$

CHAPTER III: METHODOLOGY 53

Methodology $\quad 54$

Context of the Study 55

$\begin{array}{ll}\text { Data Collection } & 57\end{array}$

Participants $\quad 59$

$\begin{array}{ll}\text { Data Analysis } & 65\end{array}$

$\begin{array}{ll}\text { Credibility and Transferability } & 69\end{array}$ 
$\begin{array}{ll}\text { Limitations } & 71\end{array}$

$\begin{array}{ll}\text { Summary } & 73\end{array}$

CHAPTER IV: FINDINGS

$\begin{array}{ll}\text { The Plan and the Purpose: PBIS Perspectives } & 76\end{array}$

$\begin{array}{ll}\text { Systemic Understanding of PBIS } & 79\end{array}$

Leadership Perspectives $\quad 80$

Perspectives of Classified Support Staff 83

Active Participation in PBIS $\quad 88$

Leadership Perspectives 88

Perspectives of Classified Staff 90

Collective Action: Self-Efficacy and Collective Efficacy 93

Leadership Perspectives 93

Perspectives of Classified Staff 96

$\begin{array}{ll}\text { Reflective Practices } & 98\end{array}$

$\begin{array}{ll}\text { Leadership Perspectives } & 99\end{array}$

Perspectives of Classified Staff 101

$\begin{array}{ll}\text { Survey Findings } & 103\end{array}$

District Level Survey Documents 107

Perspectives of Inclusion: Emerging Themes 114

$\begin{array}{ll}\text { Relationship Building } & 115\end{array}$

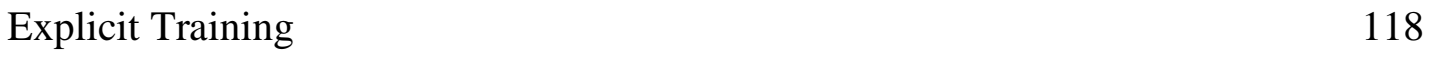

Theory in Action-Normalization Process Theory 126

$\begin{array}{ll}\text { Coherence } & 127\end{array}$

$\begin{array}{ll}\text { Cognitive Participation } & 128\end{array}$

Collective Action $\quad 129$

$\begin{array}{ll}\text { Reflexive Monitoring } & 130\end{array}$

CHAPTER V: DISCUSSION AND IMPLICATIONS 135

Discussion and Interpretation of Findings 137

Recommendations for Policy and Practice 143

Implications for Future Research 146

$\begin{array}{ll}\text { Conclusion } & 148\end{array}$

$\begin{array}{ll}\text { REFERENCES } & 150\end{array}$ 
APPENDIX A: INFORMED CONSENT DOCUMENT 164

APPENDIX B: RECRUITMENT CORRESPONDENCE 169

APPENDIX C: INTERVIEW PROTOCOL 170

APPENDIX D: FOCUS GROUP PROTOCOL 176

APPENDIX E: NPT PROTOCOL 179

APPENDIX F: 2019 SELF-ASSESSMENT SURVEY (SELECTED FEATURES) 180

APPENDIX G: RESEARCHER POSITIONALITY 183

CURRICULUM VITAE 


\section{LIST OF FIGURES}

Figure 1. Data Analysis - NPT Interview and Focus Group Framework........................66 


\section{LIST OF TABLES}

Table 1. Participating Schools .................................................................................60

Table 2. Demographic Information of District and School-level Participants ..................61

Table 3. Demographic Information of Focus Group Participants....................................64

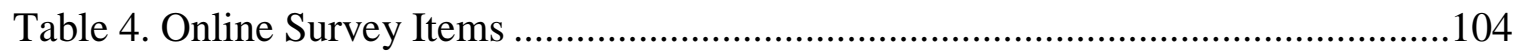

Table 5. 2017-2018 Culture and Climate Survey ......................................................108

Table 6. 2017-2018 CCS Agreement Percentages........................................................109

Table 7. 2019 SAS Overall Percentages for Featured Items .........................................112

Table 8. Coding and Themes among Leadership Participants......................................132

Table 9. Coding and Themes among Classified Staff Participants.................................133 


\section{CHAPTER I: INTRODUCTION}

Students misbehaving in classrooms is a common complaint amongst school personnel and is often cited as a reason many teachers leave the profession within the first five years of teaching (Madigan, Cross, Smolkowske, \& Strycker, 2016). Additionally, Madigan et al. (2016) note that teacher preparation programs have not effectively addressed classroom management techniques and strategies to guide new teachers. The disruptive behavior that students exhibit in classrooms often leads to punitive disciplinary actions and exclusion from classroom activities in order to remove distractions, but the research does not support the effectiveness of these practices (Gage, Whitford, \& Katsiyannis, 2018).

Schools across the country have instituted behavior intervention programs that teach students positive behaviors. Many school districts refer to these approaches as School-wide Positive Behavior Interventions and Supports (SWPBIS) or Positive Behavior Interventions and Supports (PBIS), which are "based on the logic that when students are taught positive behaviors, and those behaviors are reinforced, the overall climate of the school may improve and problem behaviors may be reduced” (Vancel, Missall, \& Bruhn, 2016, p. 320). Other school districts refer to a Multi-Tiered System of Support (MTSS) that focuses on prevention, remediation, and a continuum of supports based on types of behaviors that students display (Rodriguez, Loman, \& Borgmeier, 2016). These initiatives, if used with fidelity, provide specific strategies that schools can use to create positive supportive learning climates. 
The proactive aspects of PBIS are school-wide and the expectation is that all adults within the school setting utilize the process as agreed upon by the staff (Hill \& Flores, 2014). PBIS is not a new initiative and has evolved over the past 30 years to address concerns with the increase in negative student behavior. In 1998, under the direction of Randall Sprick, the Safe and Civil Schools developed a series of modules to assist school personnel with effective school behavior management (Sprick, Garrison, \& Howard, 1998). The goal of the modules was to provide guidance that would ensure that school personnel and students were able to have safe and civil schools.

Safe and Civil Schools published Foundations: Establishing Positive Discipline Policies in late 1990s as a tool for schools to guide their decision making on appropriate behavior management practices (Madigan et al., 2016). Providing an alternative to punitive discipline measures is widely viewed as a more effective way to handle negative student behaviors (Feuerborn \& Tyre, 2016; Jolstead et al., 2017). The decision to focus on positive disciplinary practices has evolved over time and has led to the adoption of PBIS practices in thousands of school districts in the United States. As of 2016, more than 18,000 schools are implementing PBIS (McCurdy et al., 2016).

The successful implementation of PBIS in a school is dependent on the level of staff preparation and training. This includes district-level guidance, preservice training, coaching at the school-level, and administrative support (Andreou et al., 2015; Bethune, 2017; Vancel et al., 2016). According to Filter, Sytsma, and McIntosh (2016), the largescale use of PBIS must involve all staff, and this includes teachers, administrators, and other staff in the school. 
Ward and Gersten (2013) published a report of their evaluation of the Safe and Civil Schools model for PBIS. They provided a historical context for positive behavior support that the US Congress adopted with the reauthorization of the Individuals with Disabilities Act in 2004. Within this report, they described the leadership team responsible for PBIS training as "a school administrator, at least three general education teachers, one special education teacher, and one or two other personnel” (Ward \& Gersten, 2013, p. 319). Strickland-Cohen and Horner (2015) looked at "typical school personnel" in their evaluation of behavior support planning. The focus of their study was to capture the training tools and implementation process. However, Strickland-Cohen and Horner did not clearly identify the school personnel involved in specific training beyond referring to them as team leaders and support teams.

Who are the "typical school personnel” that are responsible for providing PBIS guidance and support? Much of the literature on PBIS implementation focuses on the teacher and administrators with infrequent mention of other staff members (Feuerborn, Tyre, \& Beaudoin, 2018; Filter, Sytsma, \& McIntosh, 2016; Rodriguez, Loman, \& Borgmeier, 2016; Strickland-Cohen \& Horner, 2015). Schools employ adults whose roles reach beyond the classroom. Some of these staff members are licensed and certified staff members that includes administrators, counselors, interventionists, education consultants, and specialists. Other staff members are non-licensed and classified staff that includes assistants, office staff, and other building staff members that interact with students daily but in an entirely different manner than certified instructional staff.

In this study I focused on the other school staff members that interact with students and their involvement in the training and support of PBIS implementation. This 
included looking at school and district-level inclusion and the level of pre-employment training required for non-licensed instructional and non-instructional staff. Their specific roles and contributions to the effectiveness of PBIS implementation are defined and clarified within the study and provide insight into the vast roles these individuals play in the lives of students.

\section{Purpose of the Study}

Classified school staff work with children on a daily basis in a variety of settings. These settings include school common areas such as the cafeteria, gym, school offices, and classrooms. Non-instructional staff interact with students regularly and, at times, are a first point of contact as students begin their day. Additionally, there are instructional classified staff who work with students in classrooms, both special needs classrooms and general education classrooms. The expectations of many of these employees include providing extra academic and behavioral support to students. In order to gain a better understanding of the needs of these employees, this study examined the ways in which a large urban school district includes classified support staff in the training and implementation of Positive Behaviors Interventions and Supports.

\section{Research Questions}

To determine how classified staff receive training and support and how they are included in district initiatives, I conducted focus groups with classified staff and interviews with certified staff and district personnel. The guiding theoretical frameworks for my study included Normalization Process Theory (NPT) and organizational 
coherence (May \& Finch, 2009) to determine the effectiveness of PBIS implementation within the school district. The guiding questions include:

a) How do participants attribute meaning to PBIS and make sense of the potential outcomes for students?

b) How do participants see themselves in the action of PBIS?

c) How self-efficacious do the participants feel when involved in PBIS implementation?

d) Do participants engage in cooperative participation to ensure school-wide implementation of PBIS? If so, how?

e) How do participants reflect on their practice of PBIS data to ensure sustained implementation?

\section{Significance of the Study}

Scholars have researched PBIS extensively in the past decade in regard to implementation, staff buy-in, effectiveness of programming, and staff perceptions (Mercer, McIntosh, \& Hoselton, 2017; Jolstead et al., 2017; Rodriguez et al., 2016; Scott, 2012). Numerous measures used to quantify data regarding each of these areas are available. The most reliable measure is the School-Wide Evaluation Tool (SET), which includes a 28-item assessment given by an outside-trained evaluator. Additional measures include the Team Implementation Checklist (TIC), the Self-Assessment Survey (SAS), and the Benchmarks of Quality (BoQ) (McIntosh, Mercer, Neese, StricklandCohen, \& Hoselton, 2016). Use of these measurement tools is an efficient way to measure implementation process and fidelity and provide necessary information for 
schools and district leaders who are monitoring their own programs. Having this information enables leaders to make crucial decisions about training and support, thus allowing school staff to provide effective instruction in PBIS core systems (McIntosh et al., 2016). With this in mind, new research must move beyond these measures and start to pay more attention to the staff who are responsible for the implementation of PBIS and to seek their input. Considering all role groups will provide much needed clarity regarding the overall effectiveness of PBIS.

In two recent studies, researchers found staff buy-in was a significant factor for PBIS implementation and sustainment. However, both of these studies acknowledged that classified staff were not included in the research (Feuerborn et al., 2018; Filter et al., 2016). Filter and colleagues suggested that the level of buy-in of "non-licensed staff" needed further study due to the lack of training they received as compared to teachers and administrators. Additionally, classified school staff assist with the implementation of PBIS but they are often not included in the implementation research (Feuerborn et al., 2018).

My study built on the research of Feuerborn and colleagues and looked at how schools from a large urban school district include their classified non-instructional staff and their paraeducators in the training and implementation of PBIS. My study provides information for districts that holds implications for improved training that is inclusive of all staff who work with children, regardless of their licensure. Researchers have found a relationship between PBIS and positive school outcomes and overall school environment (Andreou, McIntosh, Ross, \& Kahn, 2015; Goodman-Scott, Hays, \& Cholewa, 2018; McIntosh et al., 2016; Rodriguez, Loman, \& Borgmeier, 2016). The focus on the 
perspectives of classified staff (both instructional and non-instructional) provides information that may be used to help advance current systems with the goal of building sustainability of PBIS implementation for the district studied and for other large urban school districts that wish to improve upon the results gained from the implementation of PBIS initiatives.

\section{Definition of Terms}

The following terms were included within the context of this study:

Social Emotional Learning: A learning process in which managing emotions, setting positive goals, showing empathy for others, and developing positive relationships is at the core of learning.

Paraeducators: School support staff who typically assist with instruction in special education and general education classrooms. These non-licensed professionals have a variety of titles that include teacher assistant, teacher aide, and instructional assistant.

Classified School Staff: School staff that are non-instructional, but who also interact with students within the school setting. These staff can include clerks, nutrition service workers, security monitors, custodians, and bus drivers.

Positive Behavior Interventions and Supports (PBIS): Positive Behavior Interventions and Supports (PBIS) is a behavior intervention system that includes individual classrooms and school-wide proactive strategies to teach positive behaviors and expectations, positive reinforcement, and prosocial behaviors. 
Tiered Interventions: Academic and behavioral interventions used to teach and assist students. Typically, there are three tiers. The first tier is for all students within a school or classroom that covers basic expectations for learning and behavior, often referred to as a universal support. The second tier is for students who need additional support or instruction due to minor or inconsistent misbehaviors that affect their ability to be successful. The third tier is for students who struggle with the additional supports provided in the second tier of instruction. These students account for a small percentage of students but require significant attention from the adults with whom they work.

Response to Interventions (RTI): a tiered approach that allows school staff to identify and support students who need academic and behavioral supports. The interventions seek to meet targeted needs, and duration of RTI is flexible depending on the ability of the student to make improvements toward success.

Multi-Tiered Systems of Support (MTSS): This system of support works in conjunction with PBIS and focuses on both behavior and academic supports for students who are not successful with the universal supports that are provided to all students within the school setting. MTSS uses multiple data points to determine if children need tiered interventions and how the interventions are implemented and monitored.

\section{Limitations and Delimitations of the Study}

Quantitative research predominates prior research of PBIS, including both large and small sample sizes. For this study, I used a qualitative methodology in order to focus on a particular group of participants and how they perceive PBIS according to their particular job roles. The delimitations of this study included the scope of participants in 
two distinct categories: those in leadership positions that were responsible for PBIS training and evaluation and classified staff members within the school setting. The classified staff invited to participate in the study served in either instructional or noninstructional positions but had direct contact with students and staff on a daily basis as part of their job responsibilities.

The case study design does not require a large sample size; therefore, I included only five schools that had a minimum of four years of implementation of PBIS as part of their core school programming. The intent of this study was to look at the perspectives of classified staff to determine the alignment between their perceptions of inclusion as compared to the perception of the district and school leadership responsible for the inclusion of PBIS in every school within the school district. Additionally, the schools selected for this study were schools recommended by the district leader who was responsible for overseeing the district mandate to ensure that all schools within the district were actively involved in PBIS implementation.

Limitations within this study account for areas that were beyond my control. One limitation was timing of the study. Focus groups took place during the fall of 2019 while school was in session. This posed a challenge when determining when I could conduct focus groups without interfering with participants' job responsibilities. School leaders at the three schools made the decision about when and where the focus groups would take place. School leaders at the elementary and middle school requested that focus groups take place during the school day. The high school focus group took place after dismissal and three of the five participants were coaches with afterschool team responsibilities, thus 
they chose to participate int the study rather than work with their teams during the focus group session.

The second limitation of the study was the selection of focus group participants. The intent of the study was to have classified staff volunteer to participate randomly in order to have a broader perspective. However, at all the focus group sessions, the administrator had asked specific staff to participate. This took away the intended randomness of the participants. At the elementary level, the administrator asked a varied group to participate (clerical, instructional, and behavioral). The middle school administrator asked her behavior support team members (home school liaison, mental health, security, and family services coordinator), and the high school administrator asked her security team to participate. All participants had the option to continue participation once I arrived and reviewed the consent forms. However, this selection of participants was a limitation of the study overall.

\section{Organization of the Study}

I organized this dissertation as follows: Chapter I includes the introduction, purpose, statement of research questions, rationale for the study, scope of the study, definition of terms, methods, data sources, and organizational summary of this study. Chapter II reviews the literature on PBIS and its historical context, current PBIS research, the roles of staff in the implementation of PBIS, and the implication of staff buy-in. Additionally, this chapter focused on the inclusion of classified staff in PBIS initiatives and the connection to theory. Chapter III is an explanation of the research methodology used, data collection, and procedures of this study. Chapter IV presents the analysis and 
results. Finally, Chapter V summarizes this study's major findings and their implications for policy, practice, and future research. 


\section{CHAPTER II: LITERATURE REVIEW}

The following research questions guided my study: a) How do participants attribute meaning to PBIS and make sense of the potential outcomes for students? b) How do participants see themselves in the action of PBIS? c) How self-efficacious do the participants feel when involved in PBIS implementation? d) Do participants engage in cognitive participation to ensure school-wide implementation of PBIS? If so, how? e) How do participants reflect on their practice of PBIS data to ensure sustained implementation? The methodology of this study provided an opportunity for each participant to provide in-depth descriptions of how PBIS influences their daily work in order to determine how this approach becomes part of their overall belief system when working with students.

The purpose of this chapter was to review the extant literature review on PBIS. I began this chapter with a brief primer and the historical context of PBIS that includes a discussion of prior research, the equity and social justice aims of PBIS, and student and educator outcomes. Secondly, I described the facilitators and barriers that affect PBIS implementation including staff buy-in, training, time, resources, and inclusion of staff role groups. Finally, I reviewed the theoretical framework used to guide this study and the implications for future research and policy. 


\section{PBIS: A Brief Primer}

Student misbehavior in schools is a significant concern amongst school staff. Studies show that a large percentage of teachers often leave the profession within their first five years of employment due to the inappropriate behaviors exhibited by many students and lack of support from school administrators (Clara, 2017; Malin \& Savolainen, 2016; Merida-Lopes \& Extremera, 2017). During the past 20 years, researchers have studied school reform efforts to address student behavior and staff responses to those behaviors. These studies have led to significant legislative changes at the local, state, and federal levels that all schools, both public and private, must follow.

Currently, more than 20,000 schools nationwide use Positive Behavior Interventions and

Supports (PBIS), a school-wide program that provides guidance for creating and maintaining a positive school environment (McCurdy et al., 2016). The PBIS framework draws upon years of research on best practices that have evolved over the years (Mercer, McIntosh, \& Hoselton, 2017; Pinkelman, McIntosh, Rasplica, Berg, \& Strickland-Cohen, 2015; Vancel et al. 2016). The framework does not require schools to purchase specific materials and allows districts and schools to determine how best to utilize PBIS based on the needs of staff and students at a particular school. PBIS is "a non-curricular, schoolbased prevention approach, which aims to promote changes in staff behavior in order to positively impact student outcomes such as student discipline, behavior, and academic outcomes” (Pas \& Bradshaw, 2012, p. 419). Pas et al. (2019) describe PBIS as follows:

The PBIS framework emphasizes the importance of data, systems, and practices as interconnected elements utilized to ensure that 
system-wide positive behavior supports can be provided to all students and that additional targeted and intensive supports can be provided to those who do not respond. PBIS schools are encouraged to use data tools to track implementation fidelity as well as student outcomes (p. 6).

Schools have found behavioral and academic success through the implementation of this framework. According to Scott (2012), schools should develop prevention plans and strategies that affect the school environment and the actions of adults by adopting proactive interventions like PBIS to help prevent common misbehaviors "from the moment students step onto school property until the moment they leave the care of their schools” (p. 191). Scott also states that adults must take ownership of this responsibility as a core belief in their roles as educators. PBIS is a framework that schools use that includes specific steps for providing Tier I supports. These include predicting problems and failures, developing effective preventions practices, being consistent with prevention efforts, and monitoring performance (Scott, 2012).

Classroom teachers and school personnel report that challenging behaviors are a major concern in classrooms and schools and they need a logical plan for addressing inappropriate behavior (Scott \& Hirn, 2013). Students must know behavioral expectations if there is to be a successful outcome and therefore these expectations must be taught. Effective teachers need to create opportunities for immediate success for students who are prone to failure through prompting, proximity, and behavior momentum (Scott \& Hirn, 2013) aligned with PBIS structures. 
Districts and schools implementing PBIS need to ensure that they have full staff commitment to proactive discipline, a set of positively stated expectations are taught, and a plan in place for rewarding and acknowledging appropriate behaviors (Mercer et al., 2017). The success of PBIS is dependent on staff participation and training prior to and during implementation (Filter et al., 2016). This review of literature will provide the historical context of PBIS, the research behind PBIS, the purpose and outcomes of the approach as they relate to student and educators. Finally, this review will highlight the roles of particular staff groups.

\section{Historical Context of PBIS}

Historically, the federal government has provided guidance on the access to education for all students through cases such as Brown v. Board of Education (1954), Rehabilitation Act of 1973, the Education for Handicapped Children Act of 1975 (EHA), Individuals with Disabilities Education Act of 1990 (IDEA), and the reauthorization of IDEA in 2004. IDEA specifically addressed discipline with guidance for decision making regarding behavior as a manifestation of a student's disability. Although IDEA addressed the need for students to have access to a free and appropriate education in the least restrictive environment, it did not address how staff should handle misbehavior in the moment.

In 2000, President Bill Clinton signed the Children's Health Act (CHA) to address eight years of research on the quality of children's health care in the United States (H.R. 106, 2000). CHA was a bipartisan act that expanded research and support for several childhood health problems. Health problems such as infant mortality, asthma, 
oral health, traumatic brain injury, and autism were some of the areas that the National Institute of Health (NIH) identified as areas in need of additional study. While these key areas addressed specific health related concerns, $\mathrm{CHA}$ also made requirements that addressed the health and safety of children in childcare centers, safe and quality mental health treatment of children in facilities, substance abuse and mental health services, youth drug use, and school safety (Freeman \& Sugai, 2013).

Within the CHA, childcare centers were required to improve the safety for children through training and educating childcare workers, improving safety standards, increasing inspections, and ensuring that childcare facilities met health and safety standards. Additionally, the Department of Health and Human Services (HHS) required that facilities report deaths of patients that had been restrained or secluded and that schools address school safety and youth violence through the Safe Schools/Healthy Students initiative (GovTrack.us, 2018). The CHA also launched an effort in both public and private institutions that required diagnosing, monitoring, and supporting children with emotional and behavioral concerns appropriately.

The CHA was an expansive act that addressed a wide range of adverse conditions that children face. According to the Clinton White House archives, the CHA legislation showed nationwide improvements in areas regarding the health and safety of students; however, there was limited regulation on how adults responded to actions deemed as disciplinary. The use of restraint and seclusion has been common within law enforcement and psychiatric institutions for years, but regulations within school settings was limited (Freeman \& Sugai, 2013). In 1998, Weiss, a reporter for the Connecticut newspaper, The Harford Courant, completed an investigation that revealed that over a 
period of ten years, 142 deaths occurred due to the use of restraints in these facilities (Weiss, 1998). One of the goals of the CHA was to address these concerns as it relates to children, but it did not go far enough to change the way states reported restraints and seclusions.

According to Freeman and Sugai (2013) two agencies conducted research that shed additional light on the use of restraints and seclusion. They noted that in 2009 the Government Accountability Office (GAO) and the Council of Parent Attorneys and Advocates (COPAA) both reported hundreds of incidents of abuse and injury of students, in particular students with disabilities. Based on these findings, U.S. Department of Education conducted a review of state regulations and policies that specifically addressed restraint and seclusion practices. Freeman and Sugai (2013) used this information in their research and found that at the time of their study only 33 states had legislation or regulations regarding restraint and seclusion.

The work of the GOA, COPAA, and the National Disability Rights Network (NDRN) shed light on the high numbers of students restrained or secluded in schools. The public outrage based on reports such as the School is Not Supposed to Hurt (NDRN, 2009) led to the introduction of legislation, the Keeping All Students Safe Act of 2009 (Hoffman, 2011). The Keeping All Students Safe Act was a proposed federal solution to eliminating the harm caused to children due to the use of restraints and seclusion practices in U.S. schools. Hoffman's review of this legislation focused on the strengths and weaknesses of the act. 
Hoffman's review of Keeping All Students Safe Act highlighted the focus on students with disabilities and the need to revise IDEA that would outline explicitly how to address these practices in Individual Education Plans (IEPs).

A brief overview of the act outlined the definition of restraint and seclusion, the statistics of their use in schools, and how the policy addressed the problem (GovTrack.us, 2011). Additional components of the act include;

1. Allowing use of physical restraints only in emergency situation;

2. Prohibiting the use of restraint/seclusion in a student's IEP;

3. Allowing the parents/families of students to pursue civil action;

4. Requiring state educational agencies (SEAs) to establish policies and procedures to promote preventative measures;

5. Requiring states to collect data and the use of restraints and to make data public;

6. Requiring schools to notify parents within 24 hours of restraint being used with their child;

7. Requiring states to establish a grant program to enhance the state's ability to provide preventative training and programming for school personnel.

The Keeping All Students Safe Act sought to provide legal protection for children in order to prevent any harmful effects from being restrained or secluded. This was done by ensuring that educational settings follow the expectations of "visual monitoring when restraint and seclusion are used, training requirements for school personnel, keeping restraint and seclusion out of special education plans, encouraging positive behavioral interventions in the school environment, requiring parental notification, and national 
reporting instances of restraint and seclusion practices” (Hoffman, 2011, p.63). A goal of the legislation was to ensure that all children who attend a public or private program that receives funds from the U.S. Department of Education were protected by this policy (H.R. 7124, 2018) (introduced).

With the recognition that punitive responses to student misbehavior are not effective, researchers like Sprick, Sprick, and Garrison began to fill a void in the educational setting. The term positive behavior supports are credited to Horner and colleagues (1990) to describe the set of strategies that would later grow to be PBIS as a systems level intervention (Ward \& Gersten, 2013). When Congress reauthorized IDEA in 2004, members referred to positive behavior support strategies as positive behavior interventions and support, and the term has been used exclusively since when referring to models or curriculum that focuses on positive strategies that support student behaviors (Ward \& Gersten, 2013).

Freeman and Sugai (2013) looked at trends in state level policies and regulations regarding restraint and seclusion. Freeman and Sugai coded states as having relevant legislation, having relevant policy or guidance, or as having no school-based guidance or legislation. In addition, they found that several states had implemented school-wide positive behavior interventions that were in line with the recommendation of Arne Duncan, the U.S. Secretary of Education in 2009, in his letter to the Chief State School Officers.

Within the body of the letter, Secretary Duncan recommended the use of Positive Behavior Interventions and Supports (PBIS) that his home state of Illinois implemented. The Department of Education offered a one-time financial resource that schools could 
apply for through the American Recovery and Reinvestment Act. The funds would provide funding for professional development, the creation of data systems, and coaching for school districts (Duncan, 2009). In 2009, there were approximately 8,000 schools in the U.S. implementing PBIS, and the U.S. Department of Education was funding the Center on Positive Behavioral Interventions and Supports website that contained information and technical assistance to schools that used the PBIS framework.

As states began to review their own legislative policies, regulations, and guidelines, the State Department of Education (DOE) of the urban school district in this study began the task of ensuring that all schools in the state addressed school climate. To support the renewed focus on positive interactions, and limited restraints and exclusionary practices, the state DOE provided the U.S. Department of Education's Resource Guide for Improving School Climate (2014) on the states' DOE website. This guiding document is available for districts to train and support their staff.

The "Guiding Principles" found on the state’s DOE website aligns with the recommendations from Secretary Duncan's push for using PBIS in schools across the country. Section one of the "Guiding Principles" document focuses on climate and prevention with an emphasis on fostering a positive school climate. The guide provides several action steps that support the work of preventing problem behaviors that interfere with student learning (U.S. Department of Education, 2014). These action steps include making deliberate efforts to create positive school environments, using evidence-based strategies, promote social and emotional learning, providing training to staff, and collaborating with outside agencies. 


\section{PBIS Research}

The empirical support for PBIS was outlined by Simonsen and Myers (2015) that included the consensus of randomized control trials that showed increases in students' prosocial behaviors and decreases in student problem behaviors when schools implemented Tier 1 school wide PBIS interventions. Results from these randomized trials set a "gold standard" in research that supported positive outcomes for schools (Simonsen \& Myers, 2015). Although there have been extensive studies of PBIS during the past decade, (e.g. Mercer et al., 2017; Nese et al., 2016) there is still a need for research that explores the factors that contribute to the sustainability of the practice (Andreou et al., 2015). The majority of the research studies use fidelity measures that are quantifiable, such as surveys, checklists, inventories, and validity scales (Mercer et al., 2017; Vancel et al., 2016). Researchers, such as Singer and Wang (2009) and Andreou et al (2015) note that qualitative methodologies can complement more commonly used quantitative methodologies. Andreou et al. (2015) recognized the need for more qualitative research designs that would help practitioners understand the actual experiences of those who are responsible for the implementation of PBIS.

While many study measures are able to quantify the implementation level of PBIS schools and districts, it is important to qualify the perceptions and lived experiences of staff. Feuerborn, Tyre, and Beaudoin (2018) used a mixed methods methodological approach to explore the concerns of classified staff. They included a qualitative analysis using the Concern-Based Adoption Model (CBAM), an established educational change theory, in order to assess their specific concerns with PBIS. This approach enabled the researchers to look at the broad feelings and thoughts that classified staff held regarding 
PBIS implementation. Feuerborn and colleagues (2018) revealed that classified staff wanted increased communication, focused on the word "positive" but failed to understand the balanced approach of PBIS, and that tensions regarding philosophical perspectives might be eliminated with explicit professional development. Additionally, they suggested future research should examine how classified staff provide input and access to training as it relates to PBIS implementation.

\section{Equity and Social Justice Aims of PBIS}

PBIS is widely used nationally and is a recommended best practice (GoodmanScott, et al., 2018). In large school districts, PBIS has shown to lower student aggression, decrease misbehavior, decrease office referrals, and decrease out of school suspensions (Goodman-Scott, Hays, \& Cholewa, 2018). PBIS focuses on teaching students what "to" do instead of focusing on what "not" to do. This enables educators to focus on the particular needs of the school and individual students. Reno, Friend, Caruthers, and Smith (2018) discussed the differences in behavior management models that often lead to harsher punishments for disabled students, students living outside of their primary homes, male students, students of color, and the socio-economically disadvantaged. Ensuring equitable responses to student behavior is necessary to meet the social and emotional needs of students (Goodman-Scott et al., 2018).

Addressing the social and emotional needs of students has become a priority in many schools around the country, and it aligns with the tenets of PBIS. According to Durlak, Weissberg, Dymnicki, Taylor, and Schellinger (2011), "Emotions can facilitate or impede children's academic engagement, work ethic, commitment, and ultimate 
school success” (p. 405). The lack of social-emotional capacities negatively influences the ability of children to develop positive relationships with caring adults in the school setting. By focusing on the social-emotional needs of students, practitioners can help shape the trajectory of a child's development leading to positive school attitudes (Taylor, Oberle, \& Durlak, 2017). Social and Emotional Learning (SEL) programs designed for school use incorporate several competencies that are necessary for children to have in order to be successful in school. These competencies include enhancing children's selfawareness, managing their emotions and behaviors, developing appropriate social skills that foster positive relationships, developing empathy and awareness of others, and making responsible choices (Durlak et al., 2011; Taylor, et al., 2017).

School interventions are instrumental in helping support positive outcomes through the integration of SEL within the classroom and throughout the entire school building. Researchers found that addressing the social-emotional needs of children creates positive personal outcomes and improves academic performance for students regardless of demographic groupings (Taylor et al., 2017). Raimundo, Marques-Pinto, and Lima (2012) found that schools who incorporate SEL programs must ensure that they are developmentally appropriate, culturally responsive, and explicit in their structure and consistency in order to be effective. Others found SEL programs had positive outcomes in all grade bands of elementary, middle, and high school (Durlak et al., 2011).

One characteristic of social-emotional learning is the acceptance and rejection of children by their peers (Torrente, Cappella, \& Watling-Neal, 2014). Torrente and colleagues found that previous research focused on deficit perspective rather than positive behaviors of children within a particular contextual setting. Instead, they wanted 
to look through a strengths-based lens to determine the factors that reinforce positive student behaviors. The researchers found that classmates liked children who expressed prosocial behaviors within a class setting. The ability to exhibit prosocial behaviors indicated that the student was adept at social-emotional competencies and therefore experienced more acceptance from peers. They found similar results in classrooms where children who lack some social-emotional competencies but had a positive teacherchild relationship found greater acceptance with their peers (Torrente et al., 2014).

An additional characteristic of SEL is the connection between social-emotional learning and executive functioning. Executive functions include directing attention, manipulating information in the working memory, and self-monitoring that allows children to control emotional and behavioral problems (DePrince, Weinzierl, \& Comps, 2009). Durlak and colleagues completed a meta-analysis in 2011 and found that a focus on SEL positively affected executive function in children through effective SEL programming led by school-based personnel. Social and emotional learning positively affects students that include partial improvement of social-emotional competencies, attitudes about self and school, and increased prosocial behaviors that supports the core components of PBIS when used in schools (Durlak et al, 2011; Raimundo, MarquesPinto, \& Lima, 2012).

Understanding how to sustain PBIS over time and the factors that help or hinder the process enables schools to determine the necessary action steps needed to support students. McIntosh and colleagues (2016) state that school demographics (i.e. race/ethnicity, free/reduced lunch status, population size, school location) play a role in successful implementation and sustainability of PBIS. In addition, researchers have also 
found that the time commitment for PBIS to be sustainable is 3 - 5 years at the elementary level and up to eight years at the middle and high school-levels (McIntosh et al., 2016).

My study has implications for future research in the areas of PBIS, fidelity measures for use with school staffs, and implications for educational policy at the local, district, and state levels. Additionally, I highlight the potential value of PBIS on decreasing the amount of disciplinary exclusions and providing evidence to support the need for high-quality experimental research that will ensure that evidence-based practices are included in the scale-up of PBIS in schools (Gage et al., 2018). In addition, this research provides evidence of how staff buy-in affects implementation and how it can be supported throughout the process of systems changes in schools (Filter et al., 2016) Additionally, considering the science of implementation as it relates to complex initiatives helps to support the evidence-based interventions that organization implement to ensure the fidelity of their use. Continued development of study measures, such as the PBIS-ACT brief (full and brief form), ensures that respondents understand the items. Two questions to consider include: what does buy-in look like in schools with lowfidelity of implementation? How does buy-in change over time or how it relates to other changes in the school over time? Future studies should create standard definitions and data collection systems to capture student behavior data and measure of time on instruction that will inform instructional policy for school based personnel. 


\section{PBIS and Student Outcomes}

Prior to the PBIS, many schools used punitive measures to handle student misbehavior, leading to exclusion from classrooms and learning (Madigan et al., 2016). To address the need for more proactive processes for handling student misbehavior, researchers developed a set of positive discipline practices for implementation at the school-level. Sprick, Sprick, and Garrison (1992) delineated these strategies in their book, Foundations. The basis of Foundations emphasized common school policies that included common area expectations, dress codes, classroom expectations, and common language through the school (Madigan et al., 2016).

The use of Positive Behavior Interventions and Supports in the large urban school district discussed in this study has evolved significantly during the past decade. PBIS falls within an overarching behavior and academic protocol referred to in the district as Multi-Tiered Student Supports (MTSS). Historically, the district provided several supports to assist schools with students who exhibited behaviors that negatively affected their academic performance and PBIS was the preferred method to ensure common goals across the entirety of the district. To ensure consistency, school expectations included creating a Student Support Team (SST) to identify students within the school who needed additional support. The team met regularly and looked at student data. They also devised plans to address student needs that were in line with the school district's guidelines.

Teachers who worked directly with the student may or may not be part of the SST, thereby creating disconnects and lack of ownership of the plan. SSTs monitored student progress through feedback from the classroom teacher to determine next steps for each plan. Data on the success of SSTs was limited with inconsistent measure and gaps 
in how plans influenced the classroom as a whole. As MTSS evolved, the inclusion of Response to Intervention (RTI) teams replaced the SST. RTI incorporated both behavioral and academic intervention plans and included direct input from the teachers, counselors, and other critical staff members through the MTSS toolkit for academics that included plans for PBIS implementation.

During this phase of MTSS in the district, schools implemented a variety of programs that leveled behaviors into three tiered categories. According to Vancel et al. (2016), Tier I supports are preventative in nature and designed for use with all students in a classroom or school. Tier II supports are for students at-risk of developing negative behaviors, and Tier III are for students who have ongoing negative behaviors and the most at-risk for developing long-term negative behaviors. Students who struggle with classroom norms and are not successful with Tier I supports are then provided additional resources that are categorized as Tier II or Tier III, if the behaviors are more frequent or extreme (Andreou, McIntosh, Ross, \& Kahn, 2015). This tiered system is a component of PBIS and research reveals evidence of its effectiveness in many school districts across the country (Gage, Whitford, \& Katsiyannis, 2018; Goodman-Scott, Hays, \& Cholewa, 2018; McCurdy et al., 2016; McIntosh, Chard, Boland, \& Horner, 2006; Simonsen \& Myers, 2015).

An example of how this might look in a typical school would be that all students participate in a class reward goal based on acceptable behaviors and following classroom and school expectations. Students who struggle with class norms and are not successful with Tier I supports are then provided with more supports and are categorized as Tier II or Tier III if the behaviors are more frequent of extreme (Andreou, McIntosh, Ross, \& 
Kahn, 2014). Tier II supports may include individual student conference, preferred seating, or parent contact. Tier III supports may include a behavior point sheet, checking in daily with a mentor adult, or alternative tasks. This tiered system is a component of SWPBIS. However, many schools still struggle to implement positive supports consistently, particularly at the classroom level (Jolstead et al, 2017).

Bunch-Crump and Lo (2017) describe PBIS as a multifaceted systems approach that includes school-wide and individual student interventions. The current usage of PBIS within this study complies with the standard protocols of tiered interventions that makes a shift to proactive and positive discipline, and commits to inclusion of all staff involved with the student(s) instead of a singular committee. The district's focus on full implementation of PBIS is part of the student handbook distributed on a yearly basis.

The overarching goal of PBIS is to address the social behaviors that affect the instructional environment of schools. Student behaviors are becoming increasingly challenging for many educators, and finding a sustainable approach to handling negative behaviors is critical to overall school environment (Andreou et al., 2015). Andreou and colleagues identified categories that would help sustain PBIS. This included continuous teaching of expectations and prosocial behaviors, positive reinforcement, working with colleagues, and having the support of school administrators.

As with SST and RTI mentioned earlier, the schools in the urban district included in this study have a core team of staff that make up the PBIS team. This team is responsible for overseeing a school-wide protocol to teach prosocial behaviors and specific criteria for each Tier. Core tenets of PBIS include full staff commitment to proactive discipline, positively stated expectations of behavior, school-wide teaching of 
the expectations, a plan for rewarding and acknowledging appropriate behaviors, a range of consequences, and analysis of school-wide data (Mercer et al., 2017).

Implementing PBIS in large urban school districts may pose additional challenges beyond the classroom that often include lower socio-economic levels, higher cultural diversity, and population density (Goodman-Scott et al., 2017). Bohanon and colleagues (2006) conducted a three-year study of PBIS implementation in one urban high school and found that by year three they still had less than $80 \%$ full implementation. The authors stated that urban schools "are often the target of large school reform efforts that are implemented without prior planning and with lack of input from staff and teachers” (p. 142). Schools, districts, and policymakers support PBIS as an effective intervention that works to decrease student misbehavior and increase students' inclusion in the classroom setting (Gage et al., 2018). However, the extensive amount of research regarding PBIS over the past 30 years shows that teachers are still concerned about the lack of support and training they receive and the need to include all school based personnel in future studies (Reno, Friend, Caruthers, \& Smith, 2018).

The phenomenon of teacher perceptions of school climate is significant according to Malinen and Savolainen (2016), who conducted a longitudinal study in Finland to investigate job satisfaction of teachers based on how negative student behavior impacts school climate and leads to teacher burnout. Another study conducted by Clara (2016) explored teacher's perspectives on adverse situations and noted that here was a high rate of teacher attrition due to difficult work conditions that included student behavior problems, constant changes in systems, and high stakes accountability. Through a case study design, Clara (2016) found that two of her three case study participants noted 
student disruptions in class and major student disruptions as a challenge that affected their emotions. Both of these studies (Malinen \& Savolainen, 2016; Clara, 2016) identified a need for more teacher education and training to address the needs of teachers. Malinen and Savolainen (2016), also address collective efficacy and self-efficacy amongst educators. Collective efficacy are the perceptions of the entire school's ability to have a positive influence an area of concern. The latter is a self-assessment of personal capacity to address an area of concern. One’s ability to manage student behavior contributes to their self-efficacy and is one of the more challenging aspects of an educator’s job (Malinen \& Savolainen, 2016). Collectively, large urban school districts are less likely to fully implement or sustain implementation as Neese et. al states, “Title 1 schools were three times more likely to abandon [SW]PBIS than non-Title 1 schools” (p. 268).

Prior research has focused on the fidelity of implementation of PBIS and the state level systems of support (Neese et al., 2016). Training and support are critical to educator perceptions of PBIS and district and school leaders should keep this in mind when structuring professional development (Vancel et al., 2016). When implemented fully, PBIS provides educators more time for teaching and higher experiences of positive impacts on problem behaviors, thus reinforcing their desire to continue implementing PBIS (Neese et al., 2016; Vancel et al., 2016).

\section{Ensuring PBIS Success}

School-based personnel play important roles in the lives of students and their ability to implement PBIS with fidelity is of the highest importance. The sustainability of 
PBIS in individual schools is dependent on the training and supports at each school. A well-trained staff is imperative to successful implementation of any initiative (Filter et al., 2016). Without proper training and support, staff will find themselves frustrated and often the initiative will fail. This is especially true in education where changes come swiftly and usually come at the behest of a mandate from higher powers from state and federal legislatures. Building capacity among school staff is crucial to implementation of any evidence-based practice (Strickland-Cohen \& Horner, 2015).

\section{Training}

Bethune (2017) conducted a study on the effects of coaching on the implementation of Tier I PBIS strategies. She found that schools needed PBIS coaches that would ensure the accuracy of core components of PBIS and that the local school should build coaching capacity within their buildings. Teachers who had direct coaching increased their level of consistency and fidelity of implementation, which had a positive impact on office referrals for student misbehavior. Bethune (2017) also stated that the inclusion of a special education teacher was important to ensure that all teachers within a building had continuous support. However, a one-time-only training was not effective. Teachers needed additional support after large group trainings or workshops. Most importantly, Bethune (2017) found that having a successful PBIS initiative could occur with the existing school personnel.

As noted by Rodriguez, Loman, and Borgmeier (2016), many schools are implementing PBIS interventions before receiving adequate training. Vancel, Missal, and Bruhn (2016) looked at how schools are implementing PBIS as it was originally designed (treatment validity) and the social significance (social validity) of the 
intervention. The authors address the need for fidelity of implementation and that school staff view the initiative as acceptable and of social importance. In addition, schools located in economically disadvantaged areas are often expected or required to engage in programs like PBIS to address district mandates (McIntosh et al., 2016). Additional district-level support for schools may ensure that schools are receiving the training and support needed for full implementation.

Districts and schools need to consider the importance of high-quality professional development in determining how to prepare staff as they implement PBIS. Desimone and Pak (2017) shared five features of effective PD: content focus, active learning, coherence, sustained duration, and collective participation. Within each of these components, the authors recommend using coaches to guide the PD in ongoing cycles that support continuous development. Facilitating factors that can enhance opportunities for PD include shared leadership, collaboration, coaching, and using research data for discussion (Moore \& Kochan, 2013). Finding time and funding for professional development is an ongoing problem in many schools, making new interventions difficult to implement (Bubb \& Earley, 2013; Pinkleman et al., 2015).

Building capacity among school staff is of the utmost importance. The collective efficacy of teachers and school leaders will determine the depth to which they commit to the tenets of PBIS and their ability to implement the initiative with fidelity (Lohrmann, Forman, Martin, \& Palmieri, 2008). All school-based personnel need to be included in ongoing training and support over the course of implementation; this includes certified and classified staff that work directly with students. The study will identify the challenges faced by schools as they work toward successful implementation of PBIS. 


\section{Staff Buy-In}

Fidelity of implementation is a key factor in whether a program is successful and meets the intended outcomes associated with the program (Mercer et al., 2017). This is true of PBIS implementation throughout schools in the U.S. Currently, there are a number of fidelity surveys that assess PBIS implementation. The survey tools seek to analyze school-wide and team effectiveness. Researchers have found that staff commitment to a program needs to be as much as $80 \%$ in order to sustain an initiative such as PBIS (Vancel et al., 2016).

To date, researchers use several survey tools to assess PBIS implementation. These include: the Schoolwide Evaluation Set (SET), an external assessment of the PBIS program based on staff and student interviews, observations, and products; the Schoolwide Benchmarks of Quality (BOC), an annual assessment combining perspectives of team members and a coach; the PBIS Self-Assessment Survey (SAS),conducted by the internal school staff; the Team Implementation Checklist (TIC), an internal evaluation that is used for progress monitoring; and the newest measure, SWPBIS Tiered Fidelity Inventory (TIF) that allows for separate assessments for each of the three tiers of PBIS. Of these assessment tools, researchers view the SET as the most objective and direct assessment due to the use of outside evaluators instead of self-assessments (Mercer et al., 2017).

The SET is the most widely used fidelity measure for PBIS (Pas et al., 2019). High fidelity is $80 \%$ or higher within the SET subscales. The seven subscales take an extensive look at the level of buy-in for each category as well as the overall measure. The SET subscales include; behavioral expectations defined, expectations taught, systems 
of rewarding behavioral expectations, systems of responding to expectations, monitoring and evaluation, management, and district-level support (Pas, et al., 2019). Pas and colleagues found that expectations taught was the subscale with the highest fidelity rankings (of $80 \%$ or above), leading them to determine that the teaching of expectations was a foundational piece for staff buy-in.

While these survey tools serve as a good measure of implementation and can provide feedback to school personnel regarding their program, there is also a need to look at the specific opportunities and barriers that arise from PBIS implementation in classrooms and schools. Mercer et al. (2017) encourages schools that are implementing PBIS to routinely assess their practice end evaluate their fidelity to the core features of the program. Staff buy-in and commitment influence the sustainability of any program and therefore feedback and staff input aid in the assessment of school-wide initiatives (Filter et al., 2016).

\section{Resources: Time and Funding}

Research shows that professional learning requires educators to spend time with colleagues in relevant and substantive trainings, often beyond their work schedule (Bubb \& Earley, 2013; Darling-Hammond \& McLaughlin, 1995; Jones, Ratcliff, Sheena, \& Hunt, 2011). Bubb and Earley (2013) state that a frequently mention barrier among educators is lack of time. Although most school districts embed training days in the school calendar, relevant professional development (PD) is not consistent (Bubb \& Earley, 2013). Effective PD needs to be ongoing, systemically planned, and coordinated in order to be effective (Jones et al., 2011). 
Districts and schools must create an environment that supports professional learning that allows for regularly scheduled times for educators to meet and learn (Jones et al., 2011), rethink schedules and staffing patterns to create blocks of time for educator development (Darling-Hammond \& McLaughlin, 1995), and include the entirety of the school personnel through adequate funding sources (Bubb \& Earley, 2013). A study by Song, Hur, and Kwon (2018) found that job-embedded PD was beneficial for developing teacher expertise and that teachers worked more productively when they were able to collaborate and attend PD offered during the school day as opposed to after their school day ended. Additionally, they found that year-round intensive coaching opportunities had positive effects on student achievement. This is consistent with Desimone and Pak's (2017) research on high-quality professional development practices.

School districts have begun to fund coaches at the school-level to ensure on-going and consistent professional development (Desimone \& Pak, 2017). Two of the five features of effective PD as listed by Desimone and Pak (2017) include sustained duration and collective participation that require schools to dedicate time and funding to support professional learning. Pinkleman et al. (2015) found that the lack of resources are in the top three barriers to PBIS implementation.

If school districts are to successfully sustain PBIS, then leadership must focus their efforts on increasing staff ownership by concentrating their effort on consistent training efforts, continuous feedback, and modeling from coaches, and providing adequate funding to ensure that time and money are available for staff support (Andreou et al., 2015). Bambara, Goh, Kern, and Caskie (2012) found that the top enablers included professional learning and development that was understood by the entire school 
staff, all school personnel fully understanding PBIS, and experiencing positive outcomes with students who had challenging behaviors. Additionally, the top ten enablers were related to beliefs, school culture, and professional development and practice (Bambara et al., 2012). Ensuring the successful implementation of PBIS is contingent on district and school leaders using research finding to address training, buy-in, and resources for all district and school staff.

\section{Classified Staff Inclusion in School Initiatives}

Public schools in the United States must provide free and appropriate educational services to all children in the country (Downing, Ryndak, \& Clark, 2000). To achieve this requirement, the use of classified staff is essential to ensure the delivery of

educational services. According to Brown and Stanton-Chapman (2017), classified staff play a major role in the educational outcomes for students, especially students with special needs. Data from 2010 show that approximately 412,498 classified staff provide supports for children in public schools (Fisher \& Pleasants, 2012). These classified staff have a variety of titles that include paraeducators, teachers' aides, paraprofessionals, instructional assistant or teacher assistants (Brown \& Stanton Chapman, 2017; Fisher \& Pleasant, 2012; Giangreco, 2013; Giangreco, Doyle, \& Suter, 2012).

For this review, I use classified staff throughout with a distinction between classified instructors (those who support teachers and schools with direct instructional practices) and classified staff (those who support students and schools outside of the academic areas) rather than the titles given in the literature to support the term used within the district of study. Classified instructors include the teacher assistants that work 
directly with the teachers in classrooms to assist in providing instructional supports. Although many of these classified staff possess various levels of educational attainment, they do not hold teaching certificates. Classified staff (for the purpose of this study) encompass school clerks, lunchroom assistants, security guards, and bus drivers that interact with students on a daily basis and provide supports in non-instructional ways.

Historically, classified staff have worked in clerical roles within schools but have shifted toward work in special education classrooms with students who need more academic and behavior support (French, 1998). The Individuals with Disabilities Act (IDEA) amendment in 1997 placed a focus on ensuring that classified staff were prepared and trained for supporting instruction in the classroom. This included an emphasis on the placement and work conditions and training in behavior management and communication skills. However, French (1998) found that training was not sufficient without close monitoring. Current research also supports the need for specific supports that will ensure the effective use of classified staff in public schools.

According to Garwood, Van Loan, and Werts (2018), "paraprofessionals are being hired to fill service delivery gaps and perform numerous roles in schools, including management of students' behaviors... in fact, paraprofessionals outnumber the total number of full-time special education teachers in schools” (p. 206). Classified instructional staff filling the gap in instructional practices can have positive or negative effects on both the students and the adults. Garwood, Van Loan and Werts also state that classified staff are emotionally exhausted due to job aspects that often include role and responsibility ambiguity, lack of respect, training and supervision, professional growth 
and opportunities, and financial compensation (Brown \& Stanton-Chapman, 2017;

Giangreco et al., 2010; Giangreco et al., 2012; Fisher \& Pleasants, 2012; Stewart, 2018).

Classified staff are important in educational settings, especially when they receive appropriate training and support (Shyman, 2010). The training and supervision of classified instructional staff is a significant factor in how classified staff feel about their jobs within school settings (McKenzie, 2011; Stockwell, 2014). Carter, O’Rourke, and Sisco (2010) found that classified staff had a limited amount of direct training or guidance from school staff and that the training they did receive often failed to connect to their actual job responsibilities. This is supported by other researchers who have studied the roles of classified staff for several years (Fisher \& Pleasant, 2012; Giangreco, Edelman, \& Broer, 2003; Shyman, 2010; Stewart, 2018).

As stated previously, several themes have emerged from the research regarding classified staff in public school settings. The first major finding focuses on the roles and responsibilities of the classified instructor. Giangreco, Suter, and Doyle (2010) found that classified instructors had poorly defined job descriptions and performed duties with limited experience. Fisher and Pleasant (2012) found that classified staff were often dissatisfied with their roles due to the feeling of marginalization and excluded from planning teams for students that they worked with. Additionally, Stewart (2018) found that job ambiguity led to poor job performance and that communicating clear roles and responsibilities to classified staff was essential to improving overall job performance.

Riggs and Mueller (2001) found that classified staff they interviewed in a mixedmethods study indicated that the majority of their time was spent providing direct instruction to students but held a wide variety of additional roles. These included clerical 
tasks, student monitoring in non-instructional areas (bus, recess, and lunchroom), assisting with projects, and behavior support. This is supported by more recent research that indicates that classified staff work in general education settings supporting behavior, teacher instruction, student supervision, communication with parents, and clerical duties (Brown \& Stanton-Chapman, 2017; Fisher \& Pleasant, 2012, Giangreco et al., 2010). Classified staff often have job descriptions, but the actual roles and responsibilities are "dictated by the supervising, lead teacher in each classroom” (Brown \& StantonChapman, 2017, p. 23).

Currently, classified instructors outnumber the total number of special education teachers in public schools in the U.S., and many of these classified instructors feel overwhelmed (Garwood et al., 2018). The work of classified instructors is often just as stressful as that of the teacher and research suggests they had a lack of self-efficacy played a role in burnout in this role group (Barnes, Cipriano, McCallops, CuccuniHarmon, \& Rivers, 2018). Studies also show that classified instructors’ most stressful responsibilities included management of student behavior, providing behavior support, and monitoring students to ensure that they were on task (Barnes et al., 2018; Downing et al., 2000; Garwood et al., 2018). Classified staff have reported that their lack of training in these areas was cause for concern (Martin \& Alborz, 2014; Riggs \& Mueller, 2001).

A second theme that emerged from the literature was the need for specific training for classified instructors. Carter, O’Rourke, and Sisco (2009) found that the most common training for classified instructors was on-the-job. They found that many schools relied heavily on informal training approaches and suggested that shared professional development opportunities would be more consistent with on-going training. Classified 
instructors often feel left out of professional development opportunities that allow them to develop in-depth understanding of specific instructional programming (McKenzie, 2011). Stockwell (2014) stated a need for intentional preparation for classified instructors that included individual coaching and direct instruction training. Classified instructors often work with students who have the most need, making it critical that they receive adequate and appropriate on-going training to support their work.

Another area of concern that emerged from the literature is the lack of compensation for classified staff, from overall salary to funding for training and development. Classified staff often have the lowest wages, and this is associated with high turnover rates (Ghere \& York-Barr, 2007). Giangreco (2013) described schools and districts that are scrutinizing higher pay and ineffectively budgeting resources to pay classified instructors for the work that they do to support better student outcomes. Many classified instructors have lower job satisfaction ratings and are particularly dissatisfied with their compensation. Brown and Stanton-Chapman (2014) found that classified staff took home \$2,200 less in monthly income than other professions in the U.S.

The themes that emerged from the literature show a systemic lack of consideration in terms of classified staff perceptions when considering their role in school wide programs and initiatives (Downing, Ryndak, \& Clark, 2000; Fisher \& Pleasants, 2012); Garwood, Van Loan, \& Werts, 2018). Feuerborn, Tyre, and Beaudoin (2018) provided additional support for this contention with a recent mixed methods study in which they examined classified staff perceptions of PBIS, finding them left out of the discussion altogether. As shown in this review, classified staff play an important supportive role in schools, yet are excluded from PBIS planning and implementation. 
According to Feuerborn et al., there is "no study in the field to date that examines the perceptions and concerns for these important stakeholders” (p. 102).

Classified instructors that support the classroom teacher often manage student behavior (Garwood et al., 2018; Shyman, 2010), yet they are often not included in ongoing PBIS trainings that are held outside of their work hours (Filter et al., 2016; Jones, Ratcliff, Sheehan, \& Hunt, 2011). Feuerborn and colleagues found that nearly half of their study participants had received little to no professional development in behavior supports and that this led to significant differences in the level of implementation for classified staff with lack of consistency found in $54 \%$ of the responses. Additionally, classified staff felt that communication was lacking along with support from teachers and administrators (Feuerborn et al., 2018).

Classified staff have unique perspectives of PBIS worthy of further examination in research studies. Supporting evidence in Feuerborn and colleagues' study indicated that lack of respect, "being talked down to, overruled, and undermined" (p.111) and lack of inclusion in training to be the major causes of concern for classified staff. The perspective of classified staff should be considered imperative due to their unique relationships with students within the classrooms and within the general school setting and should be involved in the collective school effort to support PBIS implementation. My study combines the role of classified staff and their inclusion in the implementation of school-wide PBIS. 


\section{Normalization Process Theory}

Additional research supports the importance of an organization being ready for change. May (2013) states that implementation must be understood by the organization as a process that is continuous and interactive. He discusses implementation theory and its role in developing "conceptual tools that enable researchers and practitioners to identify, describe, and explain important elements of an implementation process” (May, 2013. p. 2). May (2013) refers to four constructs of general theory: capability, capacity, potential, and contribution as they relate to implementation science. These include a framework that spells out how complex interventions move throughout an organization through the presentation of possibilities, access to resources, and actions of organization members to ensure that complex interventions are successful (May, 2013).

May (2013) and Weiner (2009) discuss the importance of understanding the conditions necessary prior to the implementation process beginning, expressing the need for individual intentions, collective action, and shared commitments as imperative components for successful change. Another aspect of implementation is the value that the members of the organization place on the new initiative or change (May, 2013). Building collective value amongst members of an organization aligns with staff buy-in and the expectations that members work together to achieve the goals of complex interventions.

Expanding on the science of implementation, May and Finch (2009) introduced Normalization Process Theory (NPT) that is designed to help us understand how practices are intertwined and become embedded into the normal practices of everyday work within an organization. May and Finch describe NPT as being "concerned with the social organization of the work (implementation), of making practices routine elements of 
everyday life (embedding), and of sustaining embedded practices in their social contexts (integration)” (p. 538). Looking at complex interventions through the lens of NPT allows researchers to carefully analyze how organizations work cohesively and engage in change.

NPT is a theory of action that looks at the social structures of an organization as they move through various levels of implementation. Within NPT are four constructs that serve as guides for explaining the social process of implementation: coherence, cognitive participation, collective action, and reflexive monitoring (May, 2009). Each construct of NPT focuses on key aspects of how organizations embed change and interventions to affect daily routines.

Ajzen (2002) explains that beliefs in consequences, normative expectations, capabilities, and intentions shape human behavior. Coherence, as defined by May and Finch (2009), is the assemblage of beliefs, behaviors, and actions that enable a complex intervention to take place within organizations. Within NPT, coherence has an additional four constructs that include differentiation, communal specification, individual specification, and internalization. Staff buy-in is about making sense of an intervention. Therefore, understanding how new practices are different from prior practices, having a shared understanding of the goals, understanding personal tasks and responsibilities, and finding value in the new practices all lead to organizational coherence (May et al., 2015).

The second construct of NPT is cognitive participation, relational work that frames practice and defines how members of an organization work together for collective action (May \& Finch, 2009). Key aspects of cognitive participation as described by May and Finch (2009) include: how the new intervention is introduced (initiation), building 
relationships amongst the groups (enrolment), organizing belief in the intervention (legitimation), and collective understanding of the action needed to sustain the intervention (activation). The cognitive participation construct of NPT accounts for the investment of the groups' commitment to an intervention.

The collective action of group members enables the actual work around an intervention. This third construct of NPT focuses on the work of interaction, accountability and confidence, skill sets, and resources, policies, and procedures (http:/normalizationprocess.org/npt-toolkit/). May and Finch (2009) posit that "production and reproduction of a practice requires that actors collectively invest in it" (p. 545). One may assume that success of interventions relies heavily on the collective action of the group.

The final construct of NPT centers on formal and informal evaluation of the implementation process by the group members. May and Finch (2009) refer to this as reflexive monitoring by May and Finch (2009). Reflexive monitoring within NPT is structured around key questions that strive to answer effectiveness, worthiness, personal relationships within the intervention's contexts, and whether procedures need to be defined or modified for improvements. (May et al., 2015).

NPT is a formal middle-range theory that explains how new ways of working are implemented in such a way that they become a part of everyday practices (May \& Finch, 2009). This theoretical framework is generalizable and can be applied in many contexts and has implications for shaping the implementation process of new initiatives and interventions, therefore it can be used in qualitative studies such as this one to analyze complex interventions in organizational settings (May, 2013). The four constructs of 
coherence, cognitive participation, collective action, and reflexive monitoring allow the researcher to look at all aspects of the complex intervention to provide a deep understanding of how initiatives and interventions work.

Research on PBIS over the past few decades have focused mainly on quantitative measures to determine if PBIS has a positive effect on student's academic and behavioral performance. Researchers have used survey tools that include validity ratings, pre- and post-test results, surveys, and multiple fidelity measures to determine effectiveness (Bethune, 2017; Bunch-Camp \& Lo, 2017; Mercer et al., 2017; Vancel et al., 2016). Some researchers used mixed-methods designs to gain some perspective from school staff but few stand-alone qualitative studies are available. Of the qualitative studies found, two stood out for their qualitative approach within large urban school districts (Bohanon et al., 2006; Goodman-Scott et al., 2018).

These qualitative researchers conducted interviews, document reviews, and field observations with a purposeful sampling of participants in bounded systems. Both studies focused on one school within a larger urban school district. Goodman-Scott and colleagues focused on an exemplary urban middle school, and Bohanon et al., focused on an urban high school. Another commonality within these two studies included implications for future research that focuses on implementation and sustainability. Additionally, these studies support the use of qualitative reviews of PBIS in urban school systems.

PBIS is a complex intervention based on the expectation that the core components of the "program" call for whole school efforts that fall within the $80 \%$ buy-in of the staff in order to show success (Vancel et al., 2016). Several theoretical frames focus on 
implementation science and provide background for how initiatives can be successful with thoughtful consideration of key areas. Weiner (2009) discusses how organizations can prepare for changes and "unfreezing existing mindsets and creating motivation for change” (p. 2). The construct of being ready for a change encompasses several levels of collective action, capabilities, and perceptions that enable an organization to begin making changes (Weiner, 2009).

The use of NPT in this study of PBIS implementation guided the focus on how the district's PBIS policies are implemented throughout the district to illustrate the nuances and variations of the policy within the general staffing population of the district. The use of this framework allowed me to categorize specific strategies and responses that positively affect the success of PBIS implementation across levels. According to Normalizationproccess.org, NPT can be a valuable method in conducting systemic reviews of organizational initiatives and can enable researchers to provide in-depth insights into the day-to-day strategies and methods employed by those asked to implement an initiative.

\section{Application in Educational Research}

One will find NPT in social science research, specifically on how routines become part of everyday work in institutional settings. As stated previously, NPT is concerned with implementation, embedding, and integration of systems within the social context. The application of NPT constructs in educational research is particularly relevant when looking at complex interventions. May and Finch (2009) provide a model for the NPT components that are transferable to education as a large system that requires collective action to successfully implement change. The first component of NPT, 
coherence, has implications for educational research due to the complexity and size of school systems and the unique managerial challenges that schools face. Fullan and Quinn (2016) provide a coherence framework for educators that aligns with NPT. The four components of this coherence framework include: focusing direction, cultivating collaborative cultures, deepening learning, and securing accountability.

Leaders of educational systems can use the coherence framework as outlined by Fullan and Quinn (2016) to inform policy, infrastructure, funding sources, and stakeholder involvement to provide whole system changes for improvement. This is consistent with the constructs of NPT and its potential use in educational research, specifically through qualitative measures. The NPT toolkit posits that researchers look at the evidence base for understanding the processes involved in implementing complex interventions, something that school systems need to consider as new and more complex interventions are introduced (May et al., 2015).

The NPT toolkit provides a range of ways that NPT could inform qualitative research: informing the focus and questions, guiding and structuring the design, sampling and data collection, guiding how data is coded and analyzed, and informing interpretations, conclusions, and interpretations (http/normalizationprocess.org/npttoolkit). This is easily transferable to educational research and provides a clear direction for qualitative studies that help leaders understand how to implement complex interventions.

\section{Application in PBIS Research}

Using NPT as a framework for PBIS research makes sense. PBIS is a complex intervention that requires staff buy-in, commitment to implementation, in-depth 
understanding of the components, and monitoring of systems (Lohrman, Forman, Martin, \& Palmireri, 2008), all of which align with NPT and implementation science (May, 2013; May \& Finch, 2009). The term normalization in this context refers to the ways in which complex interventions enmesh in the everyday practices of the workers (May \& Finch, 2009). Researchers interested in how PBIS is sustained can use this framework to gain deeper understanding by focusing on the "whys" and "how's” of successful implementation.

The four components of NPT align with PBIS as follows:

a) Coherence - how do participants attribute meaning to PBIS and make sense of the potential outcomes for students?

b) Cognitive Participation - how do participants see themselves in the action of PBIS and is there a sense of self-efficacy?

c) Collective Action - how do participants engage in collective efficacy to ensure school-wide implementation of PBIS?

d) Reflexive Monitoring - how do participants collect and make sense of PBIS data to ensure sustained implementation?

Because NPT is concerned with understanding how people perceive complex interventions within an organizational setting, using this framework for PBIS research is beneficial for understanding the implementation process and sustainability.

Future research regarding the sustainability of PBIS may include how PBIS influences educators, what supports that schools and district provide, and how teams work together (Lohrmann et al., 2008). Researchers should also shift some focus to 
understanding the factors that affect implementation and sustainability of PBIS (Bambara et al., 2012). Feuerborn and Tyre (2016) also call upon researchers to look specifically at the perceptions of staff in order to acknowledge their needs and concerns as they implement PBIS on a daily basis.

\section{Summary of Literature Review Findings and Implications for Future}

\section{Research}

This study will look at how state and local schools handle the implementation of PBIS to ensure that classified staff, both instructional and non-instructional, have the necessary training to support the core tenets of the intervention. Prior studies have shown that PBIS positively affects students, both academically and behaviorally, but there is not enough qualitative research to date in this area. Specifically, this research will focus on the role group that includes non-credentialed educators and school employees that work with and interact with children on a daily basis.

It is the intent of this study to provide an analysis that will focus on both districtlevel and school-level implications to affect new policy development. Providing firsthand accounts of how classified staff view their involvement in complex interventions will benefit district and school leadership as they develop and revise existing policy. Additionally, this study looks at the barriers that districts and schools face as they attempt to implement policy and provide an analysis of how current barriers can become enablers to ensure that all school personnel receive the support they need to implement PBIS fully.

Some areas of implication may include how classified staff leadership plan opportunities for PBIS training throughout the year. This could include training 
schedules to prepare classified staff for their roles, regardless of their particular school assignments. District-level professional development plans might look at how to create targeted components for instructional and non-instructional classified staff. Additionally, implication from this study may assist districts with establishing funding mechanisms that focus on classified staff development

For school leaders, this study may provide specific implications for PBIS as well. School leadership may be able to use information from this study to plan inclusive and ongoing training and development opportunities that include classified staff in the PLCs with certified teachers. Secondly, classified staff may become aware of ways they can increase their inclusion in the representation on PBIS committees and have an active role in decision-making. School leaders may find that this study provides support for creating opportunities for classified staff to participate in full staff meetings and trainings on a regular basis. Finally, school leaders may find this study beneficial as they work with their budget committee to allocate funding for classified staff to attend meetings and trainings beyond their workday.

Ensuring the representation of all role groups in the review of complex interventions within an organization is critical for growth and success. Therefore, this study aims to contribute implications for local, state, and federal policy as it relates to the inclusion of classified staff in PBIS implementation. It is my intent to provide a firsthand account of how classified staff are included in the training and daily implementation of PBIS. These perspectives can inform the work in other districts and schools that may be considering using PBIS as an initiative within their own districts. 
A school staff that successfully implements PBIS aids in optimizing student learning and facilitating a learning environment in which prosocial behaviors are taught and celebrated (Jolstead et al., 2017). PBIS implemented in schools can lead students to become more aware of social norms and expectations, increase time in class, decrease disciplinary referrals, and improve overall academic progress (Bunch-Crump \& Lo, 2017; Gage et al., 2018; Jolstead et al., 2017). Although many schools are encouraged to implement PBIS strategies and school-wide programs due to federal or district mandates, the positive outcomes that can result from proper implementation is significant (McIntosh et al., 2016).

My study applies the science of implementation to PBIS as called for by Filter, Systma, and McIntosh. They state that, "a focus on the science of implementation is needed to ensure that evidence-based interventions such as [PBIS] are implemented with fidelity” (p.18, 2016). Additionally, this study provides a missing piece to the implementation of PBIS regarding the perceptions of classified staff, “....we are aware of no study in the field to date that examines the perceptions and concerns of these important stakeholders” (Feuerborn et al., 2018, p.102).

It is with these foci that the study presented here has useful information to assist all levels of education to support the continued use and expansion of PBIS in schools. The recognition of classified staff in the pursuit of full implementation of PBIS provides a framework to enhance the level of staff buy-in needed to maintain current levels of implementation and to show continued growth of this complex intervention. The theoretical and conceptual framework used in this study supports the depth necessary to 
understand how every vested member of a school system can support changes that have a positive effect on the lives of students. 


\section{CHAPTER III: METHODOLOGY}

The purpose of this study was to examine the ways in which classified staff are included in the implementation of the PBIS initiative in Frederick County School District $^{1}$ (FCSD), a large urban school district in the mid-western United States. This study included the perceptions that classified staff have regarding their training and support around the implementation of PBIS strategies within the district and at the local school-level. Additionally, this study sheds light on the barriers and enablers that district and schools face as they attempt to train all staff on the complexity of PBIS interventions. By bridging the gap between the perceptions and the realities of PBIS implementation by different stakeholders, I sought to bring to light new insights that will inform the implementation of PBIS within FCSD and in similar large urban school districts. In so doing, these insights may improve implementation and increase the likelihood of obtaining desired behavioral and achievement outcomes. As stated in Chapter I, there is a lack of inclusion of classified staff in PBIS research and therefore the following research questions guided this study:

1. How do participants attribute meaning to PBIS and make sense of the potential outcomes for students?

2. How do participants see themselves in the action of PBIS?

3. How self-efficacious do the participants feel when involved in PBIS implementation?

\footnotetext{
${ }^{1}$ FCSD is a pseudonym for this large urban mid-western school district
} 
4. Do participants engage in collective efficacy to ensure school-wide implementation of PBIS? If so, how?

5. How do participants reflect on their practice of PBIS data to ensure sustained implementation?

In this chapter, I describe the research design and ethical considerations therein. I discuss the context of the study and its participants. I then discuss data collection and data analysis procedures. I also discuss my efforts to ensure credibility, transferability, dependability, and confirmability of the research study. Lastly, I discuss the limitations and delimitations of my study.

\section{Methodology}

In this study, I undertook a qualitative case study methodology in which members of district-level staff administrators, school based administrators, and classified staff participated in individual interviews and focus groups discussions. Case study research is used when the researcher seeks to answer "how" and "why" questions in areas where there is limited control over events and when the researcher plans to interpret or analyze a common phenomenon in a real-life situation (Stjelja, 2013). Yin (2018) recognizes what he calls a foundational trilogy; case study research as a mode of inquiry, case studies as a research method, and cases as the unit in the case study. He cautions researchers to be wary of research versus non-research due to the increase in case study writing that is common in the everyday writing found in newspapers, magazines, and social media outlets. Those who want to do case study research must follow a clear methodological 
plan, set high expectations and parameters, and acknowledge strengths and limitations of the design (Yin, 2018).

Specifically, I conducted a multi-case embedded case study design in which the aforementioned sub-groups provided information about variations of PBIS program design and components that influenced participants' understanding of the initiative. Each sub-group represented one of three specific layers within the large urban school district; district-level administration, school-level administration, and classified staff. To ensure clarity within this case study design, I define each sub-group within the context of the study to determine the scope of my data collection and the context between my case, the research questions, and the propositions (Yin, 2018).

\section{Context of the Study}

This study explored the nuances between district-level, school-level, and individuals as it pertains to the implementation of PBIS within FCSD. This district serves approximately 100,000 students and has a staff of over 6,000 employees. The district comprises approximately 170 schools that serve preschool through high school students. Student demographics include over 50\% minority students, over $60 \%$ free and reduced lunch, and over 30\% students with disabilities. Student discipline data over the past five years showed a decline in student suspensions. FCSD moved to a more proactive approach in regard to discipline several years ago, but evolved through several iterations of what the approach should look like and entail. Additionally, this district 
welcomed several research-based interventions that have held different meanings for each grade band ${ }^{2}$.

Prior to the district’s focus on PBIS, other research-based programs were adopted and implemented. These included Champs and Foundations, as part of Safe and Civil Schools (Ward \& Gersten, 2013), CARE for Kids, as part of Origins Developmental Design established in 2004, and the first iteration of PBIS in 2011. The district also worked within the professional development department to sponsor several texts that supported positive teacher-student interactions. Some of these texts included Teaching with Love and Logic (Fay \& Funk, 1995), We Can't Teach What We Don't Know (Howard, 2006), and What Great Teachers Do Differently (Whitaker, 2002). The district supported these initiatives and speak to the efforts of the district to develop ways to ensure that students were treated with dignity and respect.

The district also collaborated with a local university to assist with training for PBIS. Currently, all staff participate in an initial training once per year through video vignettes produced by the university. Additionally, all schools are expected to develop a PBIS plan as part of the new district mandate to implement PBIS district-wide. Within this context, I explored how staff at all levels (district, school, and individuals) worked together to ensure the full implementation of PBIS and how the intervention worked within the normal process of the organization to ensure ongoing progress.

To provide a larger perspective of PBIS in FCSD, I worked with the district's internal review board to determine which district-level administrators could provide the

\footnotetext{
${ }^{2}$ Grade band refers to a grouping of grade levels. Elementary grade band includes grades Pre-K- 5 . Middle grade band includes grades 6-8. High school grade band includes grades 9-12.
} 
most information about PBIS at the district-level. The review board also wanted me to work directly with that district-level administrator to determine which schools to include in the study. Within their constraints, I was able to reach out to six schools (two elementary, two middle, and two high); however, only five of the schools were willing to participate in the study. One elementary school chose not to participate due to other commitments they had already made to other researchers. The district gave me access to data that allowed me to work with the coordinator to select schools purposefully that had indicated either high or low levels of PBIS implementation based on behavior trend data. With this data, I selected schools that represented various demographic areas within the district. The five schools represented various areas of the community, including middleincome, upper-middle/high income, and low-income neighborhoods.

\section{Data Collection}

In order to understand the complexity of the policy implementation, I conducted individual interviews and focus groups and collected supporting documentation. The following steps describe the selection process for participants. First, I sought district leaders from within the PBIS department (See Figure 1) to conduct interviews regarding the district perspective on PBIS and staff training expectations. I interviewed two districtlevel leaders within the PBIS department. They provided a broad context of district-level expectations, goals, resources, and personnel information regarding the systematic efforts to implement PBIS throughout the district.

Secondly, I sent out emails to principals of schools that have high levels of PBIS implementation and those that have schools with low levels of PBIS implementation 
based on district designations and input from the Behavior Support Coordinator. I asked principals (or their designee) to participate in an interview and to provide names of classified staff members who might be willing to participate in a focus group. Following Lambert and Liselle (2007), I used focus groups and individual interviews to enhance my qualitative study. Using these two methods of data collections can be beneficial, as "their combination can be advantageous to researchers as complementary views of the phenomenon can be generated” (Lambert \& Liselle, 2007, p. 230). Kitzinger and Barbour (1999) prefer focus groups that consist of no more than eight participants in sociological studies as this allows for more interaction between participants. Each of the focus groups involved in this study included between four and five participants. Throughout the discussions participants engaged with each other and offered affirmations of each other.

Prior to conducting the focus groups, I sent an online open-response survey that I created using Google Forms to participating school leaders to forward to all of their classified staff members. The purpose of including this online survey was to solicit additional classified staff responses beyond just the focus group participants. The survey was live for two months, and I sent one reminder to each participating school after a few weeks to generate more responses. The survey was anonymous and only asked for email or contact information if they wanted to participate in the focus group sessions.

Lastly, I structured interview and focus group protocols to connect to the four components of NPT: coherence, cognitive participation, collective action, and reflexive monitoring. Both interviews and focus groups included semi-structured questions with opportunities for open-ended clarifying questions (See Appendices C and D). However, 
all questions sought to gain further understanding of how each sub-group views PBIS as a district-wide intervention. Additionally, questions sought to ascertain information about the roles, expectations, and understanding of classified staff as part of PBIS implementation.

Additionally I collected school-level documentation that included results from the Self-Assessment Survey (see Appendix H), school-level suspension data and Culture and Climate Survey data. I analyzed these documents in order to look at staff perceptions of climate-based structures used in each building that included PBIS. The survey documents collected responses from all staff members with only the Culture and Climate survey disaggregating classified responses separately. Looking at suspension and disciplinary referral data allowed me to analyze the level of implementation reported by the school.

\section{Participants}

Because this is a district-wide case study, participants included district leadership from central office, school-level leaders that included principals, assistant principals, and teacher leaders, and finally, classified staff such as teacher assistants, clerks, monitors, and school security. Interviews were appropriate for leadership due to their role as decision makers at the district and school-levels. The online survey and focus groups for classified staff served as an opportunity to gather the perceptions of this underrepresented role group. This allowed me to gain more insight than would be possible if I had only interviewed individual classified staff (See Appendix D). Gill, Stewart, Treasure, and Chadwick (2008) caution researchers to consider the composition of focus groups 
carefully to ensure quality discussions. This includes being mindful of how groups may interact when they are part of a pre-existing group or a stranger group and what type of discussion the researcher is hoping to facilitate.

Table 1 lists the schools that participated in the study. Each of these schools represents a different area of the school district. Starfield Elementary is located in the southern part of the district and serves a small neighborhood community considered low to middle income, as evidenced by the percentage of student participating in the free/reduced meals program. Two middle schools participated in the study. These were Kingstown Middle, which is located in the eastern part of the school district, and has students that represent all socio-economic levels but is located in an upper-middle class neighborhood. Fairview Middle also has students that cover all socio-economic levels; however, this school sits in a lower- income community in the southern part of the city.

Two high schools participated in this study. The first was Ashford Academy, which is located in the urban heart of FCSD and serves a mostly low-income group of students. The second was Woodrow Wilson High School, which is also centrally located within FCSD and serves students of all socio-economic levels. However, the neighborhood in which it sits is a middle to upper-middle income community. All of these schools draw students from across the district as part of the districts diversity plan to ensure that schools have student representation from all ethnic and financial backgrounds.

Table 1. Participating Schools

\begin{tabular}{|c|c|c|c|c|c|c|c|}
\hline \multirow{2}{*}{ Level } & School & $\begin{array}{c}\text { Enrollmen } \\
\text { t }\end{array}$ & $\begin{array}{c}\text { \% } \\
\text { Minorit }\end{array}$ & $\begin{array}{c}\% \\
\text { EC }\end{array}$ & $\begin{array}{c}\% \\
\text { ESL }\end{array}$ & $\begin{array}{c}\text { FR } \\
\text { L }\end{array}$ & $\begin{array}{c}\text { \#Classifie } \\
\text { d Staff }\end{array}$ \\
\hline
\end{tabular}




\begin{tabular}{|c|c|c|c|c|c|c|c|}
\hline $\begin{array}{c}\text { Elementar } \\
\mathbf{y}\end{array}$ & Starfield & 333 & 33 & 17 & 33 & 78.7 & 30 \\
\hline Middle & Fairview & 447 & 44 & 18 & 3 & 85.7 & 15 \\
\hline Middle & $\begin{array}{c}\text { Kingstow } \\
\text { n }\end{array}$ & 947 & 38 & 13 & 2 & 52.6 & 23 \\
\hline High & $\begin{array}{c}\text { Ashford } \\
\text { Academy }\end{array}$ & 403 & 56 & 23 & $\begin{array}{c}\text { N/ } \\
\text { A }\end{array}$ & 86.1 & 35 \\
\hline High & $\begin{array}{c}\text { Woodrow } \\
\text { Wilson }\end{array}$ & 924 & 53 & 14 & 18 & 72.3 & 45 \\
\hline
\end{tabular}

Once district leadership and schools agreed to participate, interviews and focus group sessions were scheduled. In all, the study included 21 individuals who detailed first-hand accounts of their perceptions of PBIS within their role group. Additionally, twelve participants participated in the on-line survey I provided with only one overlapping participant. Table 2 includes the district and school-level administrators that participated in the study.

The first district-level interview conducted was with Sarah who serves as the district's Behavior Support Coordinator and reports directly to the Executive Director. In her role, she oversees the PBIS training for the entire district. She has several direct reports who assist her in organizing professional development throughout the school year. Sarah has served in a district-level role for three years within this particular department, but this is her first year as the PBIS coordinator. Prior to that, she worked as a teacher in one of the district's high schools. I interviewed her at the request of the Executive Director due to her role as the lead for PBIS implementation and programming.

Table 2. Demographic Information of District and School-level Participants

\begin{tabular}{|r|c|l|l|c|}
\hline Name & Race/Gender & \multicolumn{1}{|c|}{ Position } & Years in Position & Level \\
\hline Sarah & White/Female & $\begin{array}{l}\text { Program } \\
\text { Coordinator }\end{array}$ & 1 & District \\
\hline
\end{tabular}




\begin{tabular}{|r|l|l|l|l|}
\hline Yvonne & White/Female & $\begin{array}{l}\text { Program } \\
\text { Specialist }\end{array}$ & 7 & District \\
\hline Candace & Black/Female & $\begin{array}{l}\text { Assistant } \\
\text { Principal }\end{array}$ & 12 & $\begin{array}{l}\text { Kingstown } \\
\text { Middle }\end{array}$ \\
\hline Beth & White/Female & $\begin{array}{l}\text { Assistant } \\
\text { Principal }\end{array}$ & 5 & $\begin{array}{l}\text { Starfield } \\
\text { Elementary }\end{array}$ \\
\hline Kristen & Black/Female & Principal & 5 & $\begin{array}{l}\text { Woodrow } \\
\text { Wilson High } \\
\text { School }\end{array}$ \\
\hline Frances & Black/Female & Principal & 8 & $\begin{array}{l}\text { Ashford } \\
\text { Academy }\end{array}$ \\
\hline
\end{tabular}

The second district-level interview was with Yvonne, a district PBIS trainer who works in Sarah’s department. In her role, Yvonne works with multiple schools as a PBIS consultant and trainer. Additionally, she conducts district-wide professional development to ensure that all schools have the necessary support and resources to implement PBIS at the local schools. Yvonne has worked with the district for seven years and has been a teacher and a counselor prior to her current role. These interviews were necessary to gain insight into the district's perspective on the PBIS initiative.

School leaders that participated in the study included Beth, an elementary Assistant Principal at Starfield Elementary School, with five years of experience in her position. She serves as the PBIS facilitator in her building and has a background in special education as both a teacher and consultant that she felt gave her a good background in behavior [strategies]. Starfield ES is located in a small neighborhood community considered low to middle income.

I interviewed two middle school administrators representing two distinct areas of town. Frances is the Principal at Fairview Middle School, located in a part of the city 
that has seen years of economic distress but has a great sense of community pride. Frances has been the principal for eight years. Prior to her position at Fairview, Frances served as an assistant principal and math teacher. All of her experience has been in lowperforming schools that have been through leadership and performance audits. She feels that this has helped her to understand the various dynamics of high academic standards intertwined with the need for high behavioral expectations. Candace has been an Assistant Principal for 12 years at Kingstown Middle School. Prior to her current position Candace was a special education teacher. Kingstown MS is located in an affluent part of town but does draw students from more impoverished parts of the city due to the districts diversity plan. Candace oversees the PBIS team at Kingstown.

The two high school leaders represented two distinct areas of the city as well. Kristen is the principal at Ashford Academy. She has a lengthy background in education although she has only been in her current position for two years. Kristen has been a teacher, an assistant principal, a principal in another district, and has served as a state educator to assist low performing schools. Ashford Academy is located in a highly impoverished area of town and witnessed several years of low performance and changes in leadership.

Gwen is a $9^{\text {th }}$ grade English teacher in the role of PBIS [or Behavior Support Services] lead at Woodrow Wilson High School. Gwen has been a teacher for six years and this is her first leadership role. Woodrow Wilson is located in a well-established area of town near shopping malls, large retail areas, and single-family homes of middle to high-income residents. With the district's diversity plan, Woodrow Wilson does draw many students from more impoverished areas of town (See Table 2). 
The principal (or designee) selected the participants in the focus groups. Participants were selected based on their various roles within the building and within PBIS implementation according to the school leadership. The elementary and middle school groups participants represented both instructional and non-instructional classified staff. Additionally, the participants in these two groups represented multiple demographics, such as race and gender. The high school focus group consisted of an allmale security panel (See Table 3).

The ability to meet with classified staff posed a challenge due to timing. Time included the time of year that the research study began and the time that classified staff worked. In order to reduce the negative aspects of time, I offered an online openresponse survey that included four questions regarding student discipline and PBIS. The intent was to honor the time of the classified staff as well as provide additional information from a larger sample size. I proliferated the survey to all five schools whose school leaders I interviewed.

Once I sent out the survey, I invited the same schools to participate in a focus group to delve deeper into PBIS implementation and the specific role of classified staff in the initiative. Time, once again, played a role in my ability to conduct random focus groups. I reached out to three school-leaders (one at each grade band) to assist in gaining access to their classified staff that would be willing to participate in a focus group. This allowed me to have a focus group from three schools: one elementary school, one middle school, and one high school.

Table 3. Demographic Information of Focus Group Participants 


\begin{tabular}{|c|c|c|c|c|c|}
\hline Group & Name & Race/Gender & Position & $\begin{array}{l}\text { Years in } \\
\text { Position }\end{array}$ & Level \\
\hline \multirow[t]{5}{*}{ Group 1} & Rhonda & White/Female & Bookkeeper & 1 & Elementary \\
\hline & Susanne & White/Female & Secretary & 5 & Elementary \\
\hline & Debbie & Hispanic/Female & ESL & 5 & Elementary \\
\hline & Connie & White/Female & LOA & 4 & Elementary \\
\hline & Diane & White/Female & IA & 1 & Elementary \\
\hline \multirow[t]{5}{*}{ Group 2} & Ronnie & Black/Male & Security & 4 & High \\
\hline & Micah & Black/Male & Security/Reception & 6 & High \\
\hline & Lawrence & Black/Male & Security/Coach & 18 & High \\
\hline & Rodney & Black/Male & Security/Coach & 4 & High \\
\hline & Steve & Black/Male & Security & 4 & High \\
\hline \multirow[t]{4}{*}{ Group 3} & John & Black/Male & Instructor III & 8 & Middle \\
\hline & Deborah & Black/Female & Security/Bus Driver & 15 & Middle \\
\hline & Leslie & Black/Female & $\begin{array}{l}\text { Mental Health } \\
\text { Practitioner }\end{array}$ & 3 & Middle \\
\hline & Robert & Black/Male & $\begin{array}{l}\text { Youth Service } \\
\text { Coordinator }\end{array}$ & 14 & Middle \\
\hline
\end{tabular}

\section{Data Analysis}

Qualitative data focuses on the lived experiences of study participants and allows researchers to provide detailed descriptions of specific cases and contexts (Miles et al., 2014). While qualitative data analysis can take on many forms, there are some common features found in the process that Miles, Huberman, and Saldana (2014) highlight. These include assigning codes and themes, finding patterns, phrasing, and common sequences, researcher reflections in jottings, analytic memos, and field notes, and comparing generalizations in the form of constructs or theories. To strengthen qualitative data analysis, the researcher must follow analytical processes that include data condensation, data displays, and developing and verifying conclusions (Miles et al., 2014).

My coding procedures were initially theory-driven. I used the Normalization Process Theory (NPT) as a basis for my conceptual framework (See Figure 1). This 
guided my research study and focus on key factors, variables, relationships, and constructs (Miles et al., 2014).

Figure 1. Data Analysis - NPT Interview and Focus Group Framework

\begin{tabular}{|l|l|}
\hline Theme & Code \\
\hline Coherence & $\begin{array}{l}\text { Shared Understanding } \\
\text { Roles and Responsibilities } \\
\text { Value and Beliefs }\end{array}$ \\
\hline Cognitive Participation & $\begin{array}{l}\text { Key Leaders Identified } \\
\text { Demonstration of “buy-in” } \\
\text { Involvement }\end{array}$ \\
\hline Collective Action & $\begin{array}{l}\text { Interactions between sub-groups } \\
\text { Knowledge base } \\
\text { Integration within daily work }\end{array}$ \\
\hline Reflexive Monitoring & $\begin{array}{l}\text { Effectiveness } \\
\text { Evaluation } \\
\text { Application } \\
\text { Feedback }\end{array}$ \\
\hline
\end{tabular}

NPT is concerned with the social organization of work and how it frames the implementation of practices, making the work routine (May \& Finch, 2009). NPT offers a rigorous conceptual framework for qualitative researchers and can enable researchers to analyze complex interventions in organizational settings. Use of this conceptual framework guided the interview questions and study protocols to ensure that research was theoretically sound and contributed to the scholarly literature for use in future policy making and practice.

The process of analyzing the data collected in this study began with the core tenets of the NPT framework: coherence, cognitive participation, collective action, and reflexive monitoring that provided a baseline of deductive coding. Taking the finding through these four core principles enabled me to identify areas within each tenet that either aligned with NPT or fell outside of the existing framework (See Appendix E). 
Therefore, coding began with a core set of ideas that were predetermined based on the design of the questions and variables that developed during the analysis. Throughout this process, I remained open to findings that may be outliers to the NPT constructs, understanding the concept expressed by Dyson and Genishi (2005) that “cases are constructed, not found, as researchers make decisions about how to angle their vision on places overflowing with potential stories of the human experience” (p. 2).

The first step in the data analysis consisted of a review of the interview transcripts to ensure the accuracy of the recording versus the transcript. I sent the transcripts to the interviewees for member checking; however, no participants provided any additional feedback after they received their transcripts. I conducted two rounds of coding. For the first round, I used In Vivo coding to highlight key words and phrasing that were common amongst the interviewees. I categorized the commonalities within the four components of NPT to determine where responses fit within the theory or as outliers. I then conducted a second round In Vivo coding to determine broader themes that ran throughout the interviews.

To analyze the focus group transcripts, I again used In Vivo coding for the first round to find common language within each group and across grade bands. This process included conducting a search of key words and phrases and then charting responses according to grade bands. The second round of coding consisted of looking at the grade band charts to determine themes through a more holistic approach. To get a clear understanding of the focus group recordings, I reviewed the videos to capture body language and dynamics. This enabled me to document the exact recollections of the participants in real time against the written transcript. I created memos of the video 
recordings to ensure that I maintained an accurate account of participant interactions during the discussions. Following this process, I conducted first level coding to look for patterns within the responses. I charted these according to the NPT framework and the guiding questions for the study. This allowed me to document themes across the grade bands and determine commonalities and differences within each unit.

Throughout the data analysis process, I used the display matrices and narrative descriptions to track findings and provide an orderly method to present participant perceptions. These displays included all findings, including nuances that fell outside of the NPT framework. Additionally, I subjected these matrices and descriptions to multiple cycles of inductive and deductive coding to ensure that all participant views received equal representation in the findings. The final step in the analysis of the interviews and focus groups was to review memos from those discussions to address any observances that I made outside of the transcripts that would be relevant to include in the discussion of findings.

The last area of data analysis included reviewing survey data from three documents - two from the FCSD and the one I provided to classified staff as part of the study. Self-Assessment Survey (SAS) and the Climate and Culture Survey (CCS) are district surveys distributed to schools annually. The SAS is specifically for PBIS review and conducted in the fall of each school year and the CCS is an overall climate survey distributed in the winter. For this study, I was able to pull the current school year's SAS and the previous school year's CCS. I analyzed both of these surveys for percentage of participation, percentage of agreement on key areas of climate and culture, percentages of implementation for PBIS focus areas, and percentages of improvement focus in key focus 
areas. I reached out to the school leaders following a review of the SAS and CCS analysis to determine the exact number of classified staff at each location in order to look at percentage of responses from classified staff members in order to compare it to districtlevel responses of classified staff overall.

\section{Credibility and Transferability}

Creswell (2014) discusses several ways to ensure that qualitative research is credible and transferable. In his book, "Research Design: Qualitative, Quantitative, and Mixed Methods Approaches” (2014), Creswell outlines multiple validity strategies that includes triangulation of data collected, member checking, the use of rich and thick descriptions, clarifying the bias of the researcher, and presenting counterpoints that emerge from the data analysis. Miles, Huberman, and Saldana (2014) also discuss keeping a chain of evidence of all data collected throughout the study to maintain credibility. Throughout the entirety of my study, I took multiple steps to ensure that my data were accurate and representative of participants. These steps included: collection of supporting materials from district data and interviewees, offering transcript review for all interview participants, creating and maintaining matrices of coded interview content, and allowing the perspectives of the participants to tell the story and withholding my own perspectives while reviewing all data collected.

The chain of evidence in this study included collection of transcripts from all interviews and focus group sessions. I offered study participants an opportunity to check their transcripts for clarity and provide feedback prior to the final inclusion of the transcript in the record. Additionally, focus group sessions were video- and audio 
recorded in order to capture physical actions and reactions of participants. I documented these in memos following the review of video. Careful analysis of all transcripts took place with multiple readings and reviews of each transcript. Proper documentation of participant perceptions was critical to ensure credibility and that I accurately recorded the voices of the participants.

The second portion of the chain of evidence included collection of written materials from district-level participants. This was an important means of triangulation, corroborating firsthand accounts from the interviews and focus group sessions. The survey data provided evidence as to the effectiveness of PBIS implementation within each of the participating schools. The survey documentation also allowed the inclusion of supporting evidence that demonstrated the level of participation of classified staff at both school-levels and district-level.

The final triangulation of data included the development of data displays, coding documentation, and reviewing the district's PBIS plans located on their website. All of these documents provide a full picture of PBIS implementation and the inclusion of classified staff within the initiative. This data showed the levels in which schools utilized PBIS strategies, how the staff viewed the effectiveness, and incorporated these strategies into their daily roles and responsibilities. These supporting documents also revealed the nuances between how PBIS is designed to work within the district and compare it to the realities within the participating schools.

The use of this combined data allowed me to identify structures that either helped or hindered the implementation of PBIS within this district at large and within the individual schools. Since these schools are representative of the other schools within the 
district, as the entirety of the district serves the same population of students, this information may help all schools in the implementation process. Because PBIS is currently in use in schools across the nation, the data and analysis from this study may inform practice and policy beyond the district that served as the context of my study, potentially informing the work in other districts as they make decisions regarding their own PBIS procedures.

The main purpose of this study was to reveal the perceptions that classified staff members held regarding their inclusion in the PBIS implementation process. Their voices can have far-reaching impact on leadership who wish to have full inclusion of all stakeholders when developing implementation plans for PBIS. Ensuring that classified staff members have equal access to training and support when implementing district-level initiatives is an important factor to consider and this study provides supporting evidence available for use in schools across the nation.

\section{Limitations}

Limitations in this study included access to study participants. Although I am an administrator within the district and have access to many key leaders with the district and its schools, this is not without some limitations. Direct access to cabinet level staff at central office is reserved for direct reports typically. Therefore, I anticipated having access to executive assistants in some departments and this was true in my ability to interview the Behavior Coordinator instead of the Executive Director. However, the information gathered from district-level staff provided thorough information corroborated by other study participants. The nature of qualitative case studies does not require a large 
sample size, therefore, interviewing only two district-level leaders did not hurt the study findings.

A second limitation also deals with access to classified staff needed to support this study. I did not conduct research within my own building in order to limit bias and authority issues. In order to reach other classified staff, I sought the support from other school-level administrators to recommend and assist in reaching their classified employees. These school-level leaders were able to facilitate access to their facilities for the focus groups and identify participants for focus groups at their school. Additionally, building trust is crucial with classified staff members that may still feel uncomfortable talking with administrators for fear of retaliation if they are completely honest. To address this, I discussed the confidentiality of the conversations as part of the focus group protocol and all participants would have pseudonyms to protect their identities in the final written study and in the oral defense. The goal for this portion of data collection was to gather four to eight classified instructional staff to participate in each 60-minute focus group. I was able to accomplish this target.

The third limitation was access to non-instructional classified staff who work within the school setting but not directly with students for instructional purposed (i.e. custodians, office clerks, security guards, bus drivers, and cafeteria staff). These staff members were important to the study due to their diverse interactions with students and the expectations that they interact with students in a positive manner. It was important to gain their viewpoints to provide depth to the study. Seeking non-instructional staff that were willing to participate and had the time to participate posed a challenge. To 
accomplish this goal, I worked with school-level leadership to reach out to classified staff in their buildings to seek their participation.

\section{Summary}

This chapter outlined the methods used to study how classified staff are included in the implementation of PBIS. It included the research questions, the conceptual framework, and the theoretical underpinnings that guided this study. Additionally, I outlined data collection, data analysis, and the means of addressing concerns of credibility, transferability, and confirmability. The next chapter focuses on the analysis and findings of my study. I have included summaries and details of the data I collected. The nature of qualitative studies support the use of narratives to describe the themes that emerge from interviews, focus groups, and document analysis. Additionally, I included tables and matrices that I developed to provide visual representations of the study. 


\section{CHAPTER IV: FINDINGS}

The purpose of this chapter is to provide an analysis of the implementation of PBIS as a district-based initiative. I based my findings on my analysis of interviews with educational leaders at the building- and district-level, focus groups with classified staff, survey material distributed to participating schools, and supporting resource documentation. Specifically, I focus on the perspectives of classified staff members that work in the district and how they perceive their inclusion in the PBIS initiative to ensure its effectiveness as a district-mandated program. It is the intent of this study to include the voices of an often neglected role group - the instructional and non-instructional classified staff that work with students each day to support overall school programs like PBIS.

In order to make sense of a large systematic initiative (PBIS) in a school system as large as Frederick County School District (FCSD), I applied Normalization Process Theory (NPT) as my heuristic. NPT focuses on "what people, both individuals and groups, do rather than what they believe or intend” (May et al., 2018, p. 2). Looking at PBIS implementation and inclusion of classified staff through the lens of NPT, I will address the mechanisms of coherence, cognitive participation, collective action, and reflexive monitoring which together assist in the systemic changes needed to have a successful initiative.

Here I share the findings of my study, sharing the lived experiences of participants implementing PBIS in FCSD. By sharing the story of FCSD’s PBIS journey 
to full district-wide implementation, it is my intention to provide the cooperating school district and similar districts with strategies to facilitate systemic change. This is also the telling of how staff in this school system view their roles and responsibilities as they relate to PBIS as a district-wide expectation. The voices in this study are those of individuals from various certified and classified role groups within the FCSD. They provide first-hand accounts of how they utilize PBIS on a daily basis within their role group

I organized this chapter into three sections. The first section centers on the research questions that guided my study. I sought to understand: a.) how participants attribute meaning to PBIS and make sense of the potential outcomes for students; b) how participants see themselves in the action of PBIS implementation; c) how self-efficacious the participants feel when involved in PBIS implementation; d) if and how participants engage cooperative participation to ensure school-wide implementation of PBIS; and e) how participants reflect on their practice to ensure sustained implementation.

The second section focuses on emerging themes discovered through the analysis of participants' responses and supported by survey documentation. In this section, I highlight the barriers and facilitators that influence PBIS implementation as a complex organizational initiative through first-hand accounts of those responsible for the implementation at all levels within the district. I also highlight areas of strength and growth targets that are transferable to other large urban school districts that may consider PBIS as a district-wide mandate.

In the third section, I focus on the systemic implementation of PBIS within this large urban school district through identification of strengths and areas of growth. 
Specifically, I use the NPT framework to clarify how PBIS is communicated to all stakeholders to ensure fidelity of implementation and how to support role groups that may feel disconnected from core ideology of a district as large as the one used for this study. I will also identify any findings that are outside of the NPT framework that may have influence on the successful implementation of PBIS when mandated as a districtwide initiative.

\section{The Plan and the Purpose: PBIS Perspectives}

Positive Behavior Interventions and Supports (PBIS) has been an initiative in FCSD since 2012. However, the schools involved in PBIS at that time were mainly middle schools and a few elementary schools. Prior to PBIS, elementary and middle schools utilized other behavior and classroom management programs supported by the district. These initiatives included CHAMPs, Foundations, and CARE for Kids. Between 2011 and 2018, the district changed its overall behavioral focus to be in line with researched-based PBIS that focused on school-wide common behavior expectations that teach positive behavior choices and had a less punitive disciplinary approach to dealing with unacceptable school behaviors.

Although many schools in the district were implementing PBIS, in the fall of 2018 the district mandated PBIS as a district-wide initiative at all grade bands. The district categorized each school into one of four levels that would determine the amount of training that the school would need to ensure that they had adequate PBIS strategies built into their school plans. The district made the determination for each schools level and prescribed a set plan of professional development that the school would undertake, 
beginning in the spring of 2019. The schools that participated in this study were all current PBIS schools that had been implementing for at least one school year.

Individual interviews were held in the personal offices of Sarah (Behavior Director), Candace (Assistant Principal, Kingstown MS), Frances (Principal, Fairview MS), and Kristen (Principal, Ashford Academy). The remaining interviews took place in locations outside of the schools or district offices convenient for the participants. I interviewed Emily (Assistant Principal, Starfield ES) in her home. I interviewed Gwen (Teacher Lead, Woodrow Wilson) at a local coffee shop, and I interviewed Yvonne (District Resource Teacher) in my office. The average length of the interviews was approximately 30 minutes.

I held the focus groups at the schools in which the participants worked. According to Hydén and Bülow (2003), the makeup of a focus group can influence the results of the data collection and researchers should be mindful when they review the data collected carefully to avoid skewed results. Taking the makeup of each focus group into consideration during the sessions was imperative to avoid predetermining the participant responses. I remained mindful of the fact that the participants selected to participate were at the request of the school-level administrator in order to ensure that I would not prejudge their responses.

Starfield Elementary School’s current enrollment is approximately 350 students and employs 30 classified staff members. I had the pleasure of meeting with Susanne (secretary), Rhonda (bookkeeper), Connie, (lunchroom office assistant -LOA), Debbie (bilingual instructor), and Diane, (special education instructional assistant -IA). The assistant principal asked these specific staff members to participate due to their various 
roles within the building. I was a little concerned that this would skew the information given during the focus group session, but once we got started, it was clear that they all had varied views of and experiences with PBIS.

Fairview Middle School’s student enrollment in approximately 418 students and employs 15 classified staff members. The participants in this focus group were Robert (FYS Coordinator), John (Home/School Liaison), Leslie (Mental Health Practitioner), and Deborah (security). Robert and Deborah have worked at Fairview for 14 and 13 years respectively. John has worked at Fairview for eight years and Leslie is newer to the Fairview staff and has only worked there for three years. They refer to themselves as a team, and throughout the focus group they spoke frequently about how they developed their strategies for supporting high-needs students.

Woodrow Wilson High has approximately 900 students and employs 45 classified staff. The participants in this focus group included Ronnie, Lawrence, Steve, Micah, and Rodney. All of these men serve as school security and coaches for various school teams. One participant, Keith, only participated for the first ten minutes due to a scheduling conflict. This group of men were asked to participate by their school administrator based on their direct involvement with student discipline.

Several commonalities were present during each of the focus group sessions held in this study. The first commonality was that group leaders emerged early on, and some participants remained passively engaged throughout the duration of the session. These group leaders answered the first questions and set the tone for the other participants. These group leaders stayed engaged throughout the duration of the focus group and in each session would speak up if there were lulls in the conversation. Another dynamic that 
appeared in all three groups was the support they had for each other. In all three sessions, participants acknowledged each other's comments and provided feedback and responses beyond the initial questions asked. In each session, they provided positive feedback to the speaker and offered specific examples of to support their views of PBIS.

Each interview and focus group session consisted of a set of guiding questions that focused on how PBIS normalized within their individual settings. Participants shared their perspectives on training, daily job responsibilities, and their interactions with colleagues. The remainder of this section provides the perspectives of the participants about systemic understanding of PBIS, the collective actions of staff, the cognitive aspects of participation in the initiative, and finally how participants reflect on their personal implementation practices that support the core aspects of PBIS.

\section{Systemic Understanding of PBIS}

The decision to move PBIS to a district-wide initiative in Fredrick County required a large-scale effort to develop a baseline for each school and adjust training and support to meet the needs of individual schools. To understand how FCSD worked to ensure that all schools have a shared understanding of PBIS, I asked participants to discuss how they defined PBIS and how they came to that understanding. During interviews and focus groups, the discussions revealed common threads between leadership and classified staff regarding how individual knowledge was obtained and then distributed to the group. 


\section{Leadership Perspectives}

The first person interviewed for this study was Sarah, who oversees PBIS for

FCSD. Sarah had been in this role for only a year, but she is responsible for creating the plan to revise PBIS to encompass the entire district and ensure that all schools receive appropriate and adequate training. However, this was also a learning curve for Sarah as she admitted, "I didn't even know what PBIS was when I started working in the district office.” Once she understood the core values of PBIS, she came to believe that it was “just good teaching.” As Sarah worked with her team of resource teachers, she would hear of staff in schools that did not perceive PBIS as necessary because they lacked significant behavior problems and found that their students were already compliant with expectations.

One challenge for Sarah and her resource teachers was to change the narrative of why PBIS is important in all schools. She stated, "Why wouldn’t you want to be clarifying your expectations for kids? Why wouldn’t you want to affirm when they’re doing the right thing” (Sarah, interview). The perception that PBIS is only necessary in schools with significant behavioral problems is something that Sarah attempts to address in trainings that she provides. Sarah encourages school teams to think about how they will onboard new staff and people who come into the building as guest.

I also interviewed Yvonne, a district resource teacher who works for Sarah. Her job responsibilities include leading professional development sessions on PBIS implementation at both the district level and for individual schools. Additionally, Yvonne works with seven schools as their PBIS resource teacher and assists her assigned schools with PBIS implementation strategies that meet the particular needs of the 
individual schools. When asked specifically about the inclusion of classified staff in PBIS training, both Sarah and Yvonne offer similar answers. Yvonne stated that, "They are encouraged to include classified staff, but not mandated" (Yvonne, interview). One way the school addresses this is through whole staff meetings at the beginning of each school year. All employees (certified and classified) are required to watch a series of videos about PBIS. Sarah noted that, although limited, this exposure is beneficial to all staff in order to learn basic systems and concepts.

For Sarah, her biggest challenge is to change mindsets for schools that have not bought in to PBIS while also providing adequate support to schools that are eager to have guidance. Yvonne noted that her biggest responsibility was to make sure that schools were able to move forward without her help by building their capacity. However, Yvonne acknowledged that while PBIS teams (at the individual schools) had systems in place, they often did not communicate "why they're doing what they're doing or even what they're doing at times” to all staff members within the school.

I conducted school-level interviews at one elementary school, two middle schools and two high schools. At the elementary level, Beth (Assistant Principal at Starfield Elementary) expressed that she did not think that classified staff necessarily understood what PBIS was about but that they "pick up on things they hear”. At the middle schoollevel, I conducted interviews with Candace (Assistant Principal at Kingstown MS) and Frances (Principal at Fairview MS). Both are the leaders of their PBIS teams and oversee behavior data analysis. Their combined understanding of PBIS included building proactive strategies to ensure that appropriate behaviors for students were encouraged and supported by the actions of adults. 
Frances felt that her classified staff understand the core beliefs of the PBIS strategies that she oversees at her school even if not explicitly stated as PBIS. For example, Frances noted that her Family Resource Center coordinator and Home School coordinator are extremely active in the implementation of PBIS, even if it is "not outright PBIS, but reinforcing classroom routines” (Frances, interview). Candace spoke globally regarding the school-wide culture that PBIS implementation encompassed, "more proactive instead of reactive...inclusive of teaching students how to act and what's appropriate” (Candace, interview).

At the high schools, Kristen (Principal at Ashford Academy) and Gwen (Teacher Lead at Woodrow Wilson HS) had different views on PBIS as an initiative based on the particular developmental needs of high school students within the school setting. Kristen stated that she felt that high school students exhibited different behaviors from other students and that much of what they did revolved around restorative practices. Kristen also revealed during her interview that she was unaware of PBIS prior to working at her previous location just one year prior, which had been in a middle school in FCSD.

During her interview, Gwen stated that there was "limited accountability and fidelity to [PBIS] expectations”. Gwen expressed that she felt her role was one of "checklists and compliance”. She did have prior knowledge of PBIS and understood it to be part of building culture and climate. Her overall feeling was that the classified staff received second hand information, as they were not a part of the core team charged with PBIS implementation. Gwen summed up her view of what she felt teachers at her school believed about PBIS and other district initiatives, stating, "PBIS is synonymous with 
coddling and babying and RP is synonymous with 'they don't get any consequences” (Gwen, interview).

Throughout all of the interviews, the participants in leadership roles provided few explicit examples of their classified staff interacting with colleagues on PBIS while simultaneously expressing that their classified staff were aware of the expectations for PBIS within the overall school setting. The one common thread of training amongst all of the interview participants was the overview that schools conducted at the beginning of each year that included some components of PBIS review for the entire staff. For school and district leaders that participated in this study, PBIS was a non-negotiable aspect of building a climate and culture to support students. The majority of training focused on certified staff and ensuring that teachers had support when behavioral concerns arose. However, disconnects existed between intentional planning for classified staff and an assurance that these particular staff members had a true understanding of PBIS and its core components. In all, ensuring that classified staff received training in the initiative was an afterthought for each of the leaders interviewed.

\section{Perspectives of Classified Support Staff}

The classified staff of Starfield Elementary were aware of PBIS as an initiative within their school, and all but one had attended training in previous years. When asked to explain their understanding of what PBIS meant and the expected outcomes for students, Diane (Instructional Assistant) offered the first response, "I guess I would imagine the goal is positive interventions, including positive reinforcements and things of that nature.” I asked her to clarify how she came to that understanding, she replied that 
she had "kind of pieced it together" by hearing other people talk about it [PBIS] around her even though not directly to her.

Debbie (Bilingual Instructor) added that she thought PBIS included having an expectation that every kid would have good behavior and do what they are supposed to do. Susanne (Secretary) stated that PBIS was to help give extra support. The other participants shook their heads in affirmation as each of their colleagues spoke. Debbie stated that, "Most of the time you use common sense, you know, but if we were to have more training, it would be great”. Diane once again stated that she pieced together her understanding, but she never had any formal training (at this school or her previous school).

The staff at Fairview Middle School revealed similar responses regarding their understanding of PBIS at their school and within the district at large. Leslie offered the first response by simply stating that it was positive behavior reinforcement. Robert quickly added:

That's the goal. I personally don't have a clear-cut explanation of what it is. I kind of know the goal and the direction I'm assuming that PBIS is moving in, as far as positive reinforcement, as far as giving kids a chance to correct their behavior, but I don't personally have, like a real clear understanding on how we implement that and what the... You know, I think know where they want it to end, but as far as how we get to that ending, I don't have a clear understanding of it (Robert, focus group transcript). 
John, the home-school liaison, served on the PBIS team at Fairview before the school underwent a major restructuring plan that took place three years prior. During that time, he was trained along with the PBIS team to help move away from a referral and suspension culture to a relationship building culture between teachers and students and called it "the whole positive deal” (John). Additionally, John remarked that sharing of information followed a path from administration to certified teachers and staff, and anyone else, just "catches it" was the standard flow of information whenever a new initiative came from the district. The focus on positive interactions with students was not something he learned through training of any particular program; rather, "it is who we are.”

Leslie, a classified staff member, stated that it came down to pay. She stated. “...they're cutting back on everything, especially when they took the [school resource] officers out of the buildings". John did not think that being left out of training was malicious in any way, and that they system itself is designed to be data driven on academic goals. He expressed that many of the classified staff he has worked with over the years are experienced and proven workers and that they should have opportunities to earn teaching certifications.

The final focus group session was at Woodrow Wilson High School. The participants in this session were all members of the school's security team and were not clear about the purpose of the meeting. This revelation was not overtly surprising to me based on my previous interviews with the high school leaders that suggested less compliance at the school level, even though this was a district-mandated initiative. 
Therefore, I offered a brief explanation of why I was interested in their insights regarding positive behavior interventions with students.

Once I used the specific descriptors of "positive behavior" and "interactions with students”, Keith stated that his job was to deescalate students when he had to remove them from class. He went on to say he was passionate about his work and ensuring that students had a safe environment and a place to cool down until an administrator was available. Lawrence referred to the concept of PBIS as redirecting types of strategies, conferencing, one on one counseling, and positive reinforcement. Ronnie added that he liked it because he felt that students never had the opportunity to go back to class once put out by the teachers when they made mistakes.

This team expressed limited knowledge of the specific processes involved in PBIS implementation but were able to discuss the particular requirements of their roles on the school security team and how positive reinforcement changed their interactions with students. These participants did not mention any initiative by name and only talked about the realities of their daily interactions with students and adults. What they did allude to was better outcomes for students who were able to stay in classes or return to classes to continue their instruction and that decreasing student suspensions from school was important.

From the focus groups, three themes emerged. First, all of the participants shared an understanding that the purpose of PBIS was to develop or maintain positive interactions with students. Second, all participants were able to give examples of what those positive interactions looked like. Whether this was positive reinforcements of supportive actions, giving students chances to correct behaviors, or simply providing a 
safe place for students to cool down when they were upset. Finally, it was evident that these participants had come to these understanding through second hand information that they "happened upon” and that intentional and explicit training was not consistent at any of the grade bands for classified staff.

During the focus groups, many of the participants talked about their personal actions to build relationships with students and provide supports to students that would allow teachers to continue teaching without disruption. These classified staff members measured student outcomes based on cutting down office referrals and out-of-school suspensions. The middle and high school focus groups participants stated that school administration wanted them to assist with keeping student suspensions lower by intervening when students exhibited inappropriate behavior in the schools. They did not monitor student outcomes personally but worked with administrative staff to provide a layer of support that would cut down on office discipline referrals that might lead to increased out-of-school suspensions overall.

Although focus group participants had positive views of PBIS overall, they did not have a deep knowledge base about PBIS as a research-based practice. For them, the term PBIS was something that was talked about within the school setting as a focus for student and adult interactions. However, the discussions revealed that the work these classified staff took part in on a daily basis was PBIS in action, even if they did not directly see the link when using the term. 


\section{Active Participation in PBIS}

Collective action is described as the "operational work that people do to enact a set of practices” (Lloyd et al., 2013, p.4). In order for PBIS to be sustainable in Frederick County schools, those involved in the everyday actions of implementation should be able to see themselves actively engaged in the initiative. Therefore, I asked participants to describe how they saw themselves in the action of PBIS. This line of questioning allowed me to understand their individual roles as well as how they work collectively to put PBIS strategies into everyday practices.

\section{Leadership Perspectives}

As the district's lead administrator for PBIS, Sarah drew upon survey documents and the district's trend data in determining which schools were actively participating in PBIS and which schools had stopped implementing or were not successful. She utilized these factors to develop tiered training modules to assist schools as they devised PBIS plans. Both Sarah and Yvonne stated that they are required to train all schools as part of a corrective action plan mandated by the state's department of education in order to address high levels of punitive disciplinary measures within the school district. Yvonne stated, "I have to train all the schools to be PBIS schools. I am also responsible for helping the school leads.”

At the elementary and middle school levels, Beth, Candace, and Frances all oversee the monthly PBIS team meetings, review and analyze behavior data, and make individualized plans for students who are considered Tier 2 or Tier 3, as it relates to meeting behavior expectations. All three of these school leaders were active participants 
who expressed ongoing interactions with their staffs to ensure that PBIS implementation. When asked specifically about their interactions with and inclusion of classified staff, all three stated that classified staff were either part of the [school-based] PBIS team or, at minimum, supported the certified staff by intervening with students on their behalf.

This was different at the high school level. Kristen made sure to oversee the PBIS core team that consisted of her administrative team, the counselors, resource teachers, and teacher leaders. No classified staff are included in her core team. Kristen was unsure if her classified staff were using PBIS strategies and mentioned that she has high turnover that may affect implementation. Gwen, PBIS Lead at Woodrow Wilson HS, holds a precarious position. As a teacher lead, she does not make scheduling decisions nor does she oversee training at the school-level. Gwen receives her instructions from district resource teachers and her principal.

The administrators at these schools articulated a clear vision of what they want PBIS to look like in their buildings, although they did not plan with the intention of purposely including classified staff in the process. None of the school leaders spoke about organizational structures that would encourage full implementation of PBIS from all staff members. The focus for school leaders was working with certified staff members who were responsible for academic performance. This is not surprising since district and school leaders are responsible and accountable for student performance, both academic and behavioral, and PBIS has shown to have a positive impact on both of these areas. 


\section{Perspectives of Classified Staff}

Classified staff participants were able to define their roles and responsibilities and made some connections to specific PBIS expectations. Connie (LOA at Starfield ES) explained that in her role, she tries to stay positive with the students, even when they do not always follow expectations. Diane (IA at Starfield ES) discussed strategies she used within the special education classroom to prevent escalations. These strategies included positive reinforcements, break cards, or tokens, noting, "We try to cut off behavior before it happens when we can.”

Susanne (Secretary at Starfield ES) revealed that the office staff were often mediators for the students, stating, "I think the value of it is taking the time to listen to the student as to what's going on and not push it aside...”. In addition, Susanne expressed that students would reach out to members of the office staff when they were having a bad day. This enabled the office staff to intervene with the teacher on behalf of the student if they deemed such actions necessary. In these instances, there was no direct mention of PBIS strategies, but reference to an overall view that they had the ability to build supportive relationships with students.

Debbie, who works with bilingual students at Starfield, stated that her role is to support students who may or may not want to work. Her only mention of a PBIS related behavior on her part was that she needed to be patient and do whatever she needed within a situation where a student may not be motivated. She provided encouraging words about being successful and getting a job, but she qualified that she did not really have to deal with behavioral issues. 
Several middle schools in FCSD have implemented PBIS for multiple years, including Fairview MS. The classified staff that participated in the focus group were unable to discuss specific training that they had actively participated in over the years. However, when asked about their daily interactions with students, they shared many examples of actions that aligned with PBIS core tenets when working with the student body.

John, the Student Services Coordinator, explained his process for deescalating situations between students. This involved intervening in student disagreements through mediations and problem-solving sessions with students to prevent exclusionary consequences from administrators. John stated, “[We] figure out what the problem is. If we can get it squashed, we send them back to class." John and Robert oversee the systems that cover community areas and set expectations for student behavior with some autonomy from the administration. They take the time to learn as many students by name as possible so they can engage with them personally, thus allowing them to oversee the large common areas like the cafeteria and hallways where potential misbehaviors often occur. They focus on being proactive, which is key in PBIS to ensure that students are aware of and taught expectations. Although this team serves in a support role to the certified staff, the actions they take to prevent problems from occurring has helped to keep disciplinary referrals down and return students to classrooms.

At Woodrow Wilson HS, Lawrence and Steve added that everyone on the team had different tactics they used with the students they worked with on a daily basis. In the past, they were more physically aggressive with kids, but now they worked on deescalation and talking with students instead. Both men wanted students to have 
consistent consequences for inappropriate behavior incidents and wanted the back-up of administration as well as having adequate information in order to understand the decision making process. Lawrence explained that he likes to listens to students: "Sometimes all they want to do is be heard. That's our job... and now they are calm”. Steve summed it up this way:

I also think that us as a whole, meaning security, we have a different relationship with all these kids anyway, because if you look at it, Micah is the head of ‘social group’. Lawrence coaches sports, I coach sports. He (pointing to Rodney) coaches sports. We all relate to these kids, not only in the hallway, but we get a different kind of relationship with them because we are coaches...So we can find things out, and we can relate to kids. And sometimes kids just open up to us more than they open up to anybody else. (Steve, transcript)

Ronnie expressed his love for the job and the ability to touch children's lives as confidantes and mentors. All of the participants at Woodrow Wilson engage with students inside and outside of the physical school walls and develop connections with students. As Steve explained, "I have kids mad at me every day, and I have kids tell me they love me every day.”

Overall, the focus group participants viewed themselves as active participants in school climate and safety and acknowledged this as the focus for their job responsibility. Most carry out their daily routines without questioning the why of what they are expected to do as it relates to PBIS implementation. As stated in the findings, none of the participants served as members of their school's PBIS team and did not participate in 
staff meetings where faculty received PBIS updates. These classified staff members did not express a desire to attend additional meetings but were open to the idea of participating in additional training when directly asked.

The majority of the focus group participants saw themselves as integral members of the culture and climate of the school and were often the first to respond to behavior incidents that occurred in classrooms and common areas of the schools. Classified staff respected the decisions of the administration and therefore did not question many of the actions taken to implement PBIS school-wide. Their ability to relate to the students and provide mechanisms to deescalate and calm intense situations was critical to getting students back into the classroom settings. In this way, their work is integral to PBIS implementation and its success as a district-wide initiative.

\section{Collective Action: Self-Efficacy and Collective Efficacy}

According to Bandura (2000), people are products as well as producers of their environment and, as such, they work within organizational settings and implement initiatives to the best of one's abilities. It is important to listen to the perspectives of the participants from this study in order to understand how their beliefs in their self-efficacy and as a collective group influences the success of PBIS as a district-wide initiative. In this section, I focus on the individual and group perceptions of efficacy.

\section{Leadership Perspectives}

During our interview, Sarah indicated that district's role is to build capacity within the schools and make sure that the PBIS Leads have the depth of knowledge to 
train their staff members. This included providing PBIS manuals to schools via Google Drive access and the assignment of district PBIS resource teachers to schools to assist their teams in developing school-based plans. However, this department does not mandate that classified staff are included on PBIS teams. They only recommend that classified staff be considered to serve. Because this does not directly affect the successful implementation of PBIS within a school, leadership did not express a strong opinion on this subject.

Classified staff are able to participate in two survey items that schools take during the school year, the Self-Assessment Survey (SAS) and the Tiered Fidelity Inventory (TFI); however, there is no way to disaggregate survey results by specific role groups. Additionally, the district invites classified staff to attend any school-based or districtlevel training, but the training times are often outside of the classified staff workday. Sarah does not offer guidance to school leaders that may want to adjust training to include classified staff. School-level leaders make these school-level decisions. To this end, only Kristen (principal at Ashford Academy) provided specific training for her classified staff.

All of the schools that participated in the study had a PBIS team that included various stakeholder groups, although not all of the teams included classified staff members. Each school leader was able to articulate a basic understanding of PBIS as a school-based positive intervention that influenced school culture and climate and all served as the facilitator for their core team. The depth of implementation efforts varied according to grade bands. 
Beth's team consisted of both certified and classified staff; however, specific PBIS training for classified staff was limited to beginning of the year programming when all staff return from summer vacation. Candace encourages staff to "be more proactive instead of reactive and to shift thinking... be inclusive of teaching students how to act or what's appropriate.” Frances stated that at her school they were "putting systems in place to teach replacement behaviors with the kids.” Frances meets with her PBIS core team bi-weekly, but this only includes the certified staff. Frances sees her office staff as "triage" because so many students interact with the office staff; however, they do not get training, only “moments to participate” (Frances, interview). The administrators' at all three grade levels shared that they felt that their classified staff had a unique relationship with students that played a role in PBIS implementation. Collective action at the schoollevel was more integrated. For example, Beth described teachers and their classified assistants as "good cop-bad cop" where the assistant dealt more with the students' emotional needs to deter inappropriate behavior.

As a whole, the district and school leaders expressed confidence in the level of PBIS implementation overall. They had clear action items for their individual locations and made attempts to build capacity within their staffs to ensure broad understanding of PBIS. Each school-leader oversaw their school's PBIS team and used data to make decisions about areas of improvement that would decrease disciplinary referrals and out of school suspensions. Informants representing school and district leaders perceived PBIS as an integral part of the school's culture and climate. 


\section{Perspectives of Classified Staff}

Focus group participants had a completely different perspective of self-efficacy when asked to discuss their ability to implement PBIS as part of their daily job responsibilities. Levels of efficacy shifted with each grade band as participants discussed their understanding of and actions within the PBIS framework. At the upper grades, collective-efficacy was more prominent, whereas with the elementary group (which worked in silos), their discussions were focused more on self-efficacy.

The participants at Starfield Elementary have specific roles and responsibilities that did not require them to work as a group like the middle and high school groups that participated in the study. Each member of the Starfield team had a role to either assist a particular teacher, individual students, oversee a common area, or serve in a clerical position. Therefore, they looked at PBIS through the lens of individuality. Diane was confident in her abilities based on the work she did with her collaborating teacher, stating, "For me, working with my lead teacher, like we have an open dialogue between the two of us." The rest of the participants at Starfield had strategies independent of the others within the focus group. As Connie stated to Diane, "It's good to have that. Not everybody has that rapport with someone.”

At Fairview Middle School, the participants described their particular roles and their relation to PBIS implementation. Leslie stated that she pulls students out of areas of conflict to provide support services in a therapeutic way, but when students need more, she refers them to Robert and John whom she describes as "role models" for the staff and students. Robert stated that his goal is to change the culture and climate of the school and 
focus on building relationships with students. He views John as a partner that "tag teams” with him.

Between Robert and John, they describe their belief system as providing informal interventions, mediations between students, developing hallway and cafeteria procedures, and providing a safe space for students to talk and express themselves. As John puts it, “There's nothing magical about what we do. We have bought into the fact that we're not classroom teachers. There are times when we step into areas that we are not trained in.” John described his role as "undefined” when connecting it to PBIS, "we try to give input, but we're classified, it’s FCSD mentality”.

Woodrow Wilson's security team has a slightly different set of personal expectations when it came to discussing collective efficacy. Although the group had no official checklist for PBIS implementation, all of them felt that what they did everyday was PBIS in action. John summed it up, stating, “Hey, administrator, if you're trying to change the culture of your school, you not only need to have this position, but you need to try to hire this personality to fill that position... If you're doing it based on degree or certification, you're going to lose out...”

This team expressed that teachers relied on them to intervene with difficult students. Rodney admitted that although they each had different skill sets, the security team was “all in this together”. Ronnie added that most security people at the high school-level would not use the PBIS strategies, and Lawrence thought that this was because most security team members “didn’t have a clue as far as those different strategies.” 
The Woodrow Wilson security team members were confident about the work that they did each day to support students and staff. Although they did not have any specific training in PBIS strategies, their focus was impactful in cultivating a more positive school environment. As Ronnie, explained, "I try to build relationships with kids. They know if you care. They know if you're scared of them and if you are stand-offish.” According to Steve, “A lot of students want to talk and hang out”. He also described himself as a father figure or an uncle and less of a disciplinarian.

Collective action is the shared belief that the group can achieve success. The focus group participants at the middle and high school levels articulated their collective efficacy through their discussion of how they relied on each other and collaborated as a team. At both Fairview and Woodrow Wilson, they supported each other and developed strategies that allowed them to accomplish their goal of supporting students in crises or when students needed a place to deescalate. At the elementary level, the focus group participants did not work as a team, but rather independently. However, they acknowledged their individual contributions to the PBIS components that the school adopted. Overall, the focus group participants described their individual and shared beliefs about PBIS implementation and how they incorporated their belief systems into their daily work, and most of them expressed confidence in their ability to utilize positive strategies when deescalating students who were misbehaving

\section{Reflective Practices}

The process of monitoring an initiative to ensure that all stakeholders are effectively implementing agreed upon strategies and techniques is part of the reflective 
process in NPT. In this final section, I looked at how those in leadership positions viewed their feedback loops with classified staff regarding PBIS implementation at the school level and how classified staff interpreted this feedback process. Understanding how this exchange of information is important to understanding how organizations can successfully sustain and improve complex initiatives. The initial discussion regarding feedback looks at how individuals and groups receive initial training in PBIS prior to delving into how classified staff get specific feedback on their job performance as it relates to PBIS implementation strategies.

\section{Leadership Perspectives}

District leaders, Sarah and Yvonne, analyze the SAS and the TFI surveys each year. They noticed that several schools have "really good scores on the TFI and yet their pyramid is upside down... We know teachers aren't doing PBIS, but they have their systems” (Sarah, interview, referring to the three-tiered system of the PBIS model). Where does this breakdown or disconnect occur and why? Both Sarah and Yvonne speak coherently about PBIS and the core elements and expectations of the initiative. They also provide training and support to school teams and offer individualized meetings with leads to develop action plans for schools. Yet, they find inconsistent behavior data that does not show the improvements that one would expect with full implementation of PBIS.

Although PBIS training is important, there are no mandates that classified staff have the same amount of training as the certified staff, and there is limited money in the budget to pay classified staff beyond their workday to attend regular staff meeting where the certified teachers get updates on district initiatives like PBIS. Beth recalled a training that staff attended two years prior. The training was part of restorative practices with a 
small emphasis on PBIS; however, that was not ongoing. Additionally, follow up training and feedback to classified staff, in particular, were not included in the development of professional development plans.

When asked specifically about how they trained the classified staff within their buildings, both Candace and Frances noted limited training for that role group. Similar to Beth at the elementary level, the classified staff at the middle level were involved in the beginning of year training when all staff return from summer vacation are all staff are required to view mandatory PBIS training videos. But after that, there was minimal inclusion.

At Kingstown, the classified staff were not high on the priority list when it came to training. Candace referred to this as the "white elephant in the room." Training requirements and expectations for classified staff and certified staff are different. Furthermore, budgets and scheduling at the school-level pose challenges to providing adequate support to classified staff. This led to nominal training and limited feedback throughout the school year. This emerged as a consistent pattern throughout all of the leadership interviews, evincing an overall lack of explicit directions given to classified staff that would enable them to improve their job performance.

Once initial training is completed, follow-up measures to take the pulse of how an initiative is moving along is part of reflexive monitoring in the NPT framework. May and colleagues (2018) define reflexive monitoring of an initiative as "how a practice is understood and assessed by actors implicated in it” (p.16). PBIS feedback and assessment at the district-level takes place through the utilization of surveys provided to schools twice per year that include the Self-Assessment Survey (SAS) and the Tiered Fidelity 
Inventory $(\mathrm{TFI})^{\mathrm{i}}$. Once surveys are completed, district resource teachers like Yvonne work with their assigned schools to develop action plans. Although both Sarah and Yvonne spoke about the SAS and TFI, there was no mention of these surveys during interviews with school leaders.

Each school leader that participated in the study acknowledged that they provided limited feedback to their classified staff in reference to PBIS implementation. When asked directly about this process, Beth said that she provided feedback "in the moment" and Frances stated that feedback to classified staff was informal. Kristen also stated that she provides informal feedback during debriefing sessions after a behavior incident. Feedback regarding depth of understanding and performance are a necessary component in reflexive monitoring if there is an expectation of growth and success for an initiative like PBIS.

\section{Perspectives of Classified Staff}

The findings regarding feedback were similar during focus group sessions. The participants at Starfield Elementary explained that the principal provided a weekly newsletter to staff that had "shout-outs" to recognize staff; however, this was not directly related to specific PBIS strategies, and three of the five participants stated that they forget to look at the email or read the newsletter. None of the participants indicated that they received individual or group feedback regarding PBIS implementation, even though Beth, the administrator, stated that classified staff received feedback as "mostly coaching in the moment”. 
The staff of Fairview Middle viewed feedback differently. Leslie stated that she got feedback from the students, noting, "It's really the kids, because the kids appreciate it more than anybody.” Robert added that there are times when administrators talk with them about strategies that work with particular students and that formal feedback is limited. However, certified staff often sought his input about how he approached difficult students in order to gain insight from the classified staff on how to best work with a disruptive student. The Fairview participants disclosed that none of them received consistent or explicit feedback regarding their job performance and ability to improve.

The final focus group participants at Woodrow Wilson did not indicate that they received feedback regarding their job responsibilities. They focused their responses on their interactions with students and the ways students responded to them when they were intervening in disruptive situations. They did not meet regularly with their supervising administrator nor did they receive specific feedback on their job performance. This group worked as a "self-monitoring” team that communicated with each other to enact any school-wide expectations given to them by the administrative department with the autonomy to make their own decisions regarding how to deescalate students.

Steve expressed that he wanted administration to be more upfront with the security team about student consequences, “Admin knows they have shackles from being able to do certain things and when you don't have that information it frustrates you. As Robert put it, "Nobody ever sat down and said this is how we want this to look". He continued to state that any feedback they did receive from an administrator was informal and specific to interactions with a particular student. However, that feedback was more 
about the administrators seeking help with strategies that the classified staff were using instead of the other way around.

Lloyd et al. (2013) notes that teams brought together around common goals and who monitor processes regularly have increased motivation that improves the ability to sustain a complex initiative. My data and analysis revealed that feedback on job performance for FCSD classified staff, as it related to PBIS, is minimal at best. The classified staff participants indicated throughout the focus groups that they held positive feelings about PBIS as it related to building positive relationships with student overall school culture, yet they had limited resources to improve their capacity to build on their limited knowledge base.

\section{Survey Findings}

According to Hill and Flores (2014), the proactive aspects of PBIS are schoolwide, and the expectation is that all adults within the school setting utilize the process as agreed upon by the staff. However, classified staff who are expected to assist with implementation are often not included in the implementation research when it comes to PBIS (Feuerborn et al., 2018). To expand upon the oral perspectives participants expressed during focus group discussions, I provided an on-line survey to the five participating schools that agreed to participate in the study- Starfied Elementary, Fairview Middle, Kingstown Middle and Woodrow Wilson High ${ }^{3}$ schools.

\footnotetext{
${ }^{3}$ Leadership at Woodrow Wilson HS did not distribute the survey to the classified staff
} 
The purpose of using two different formats to solicit feedback was to "hear" as many voices as possible within the case study design due to the limitations in availability to classified staff. The use of focus groups did not require a prolonged engagement with participants and the inclusion of a separate survey provided a secondary method of obtaining perceptions that allowed for complete flexibility of participants to respond when they felt comfortable and to encourage more participation. Providing the online survey as an option allowed participants to provide basic information about their roles within the school setting as it related to students and PBIS implementation at a time that was convenient for their personal schedules. The online survey (see Table 4) was anonymous, and respondents were offered the opportunity to participate in the focus groups if they wanted to provide information that is more detailed.

\section{Table 4. Online Survey Items}

\begin{tabular}{|l|l|}
\hline Item 1 & $\begin{array}{l}\text { Please provide the following information: a) Job title, b) Number of years } \\
\text { in this job, and c) grade level of current job (elementary, middle, or high } \\
\text { school) }\end{array}$ \\
\hline Item 2 & $\begin{array}{l}\text { Briefly describe your job responsibilities as they relate to interactions } \\
\text { with students during instruction and/or behavior management. }\end{array}$ \\
\hline Item 3 & $\begin{array}{l}\text { Briefly describe the behavior expectations that are in place at your school. } \\
\text { How do you feel students respond to those behavior expectations? Why? }\end{array}$ \\
\hline Item 4 & $\begin{array}{l}\text { Briefly describe your interactions with coworkers when seeking input } \\
\text { regarding student discipline. }\end{array}$ \\
\hline Item 5 & $\begin{array}{l}\text { Have you participated in training that relates to PBIS? If so, please } \\
\text { describe the training and any strategies you have used. }\end{array}$ \\
\hline
\end{tabular}

The school district has used the Google platform for much of their correspondence with staff over the past few years as a way to streamline communication. Therefore, I used Google Forms to create the survey for this study due to district familiarity with the system. The survey was available to each of the schools for a period 
of 12 weeks (the principal at Woodrow Wilson HS did not provide the survey link to her staff). Each school administrator sent the link on my behalf to their classified staff (see Appendix H). I left the survey open for responses for the duration of the focus groups to give adequate time for participants, although I did ask school administrators to send a second notice to their staff after several weeks when I noticed a limited response rate.

Once the survey window closed, the responses were uploaded to an excel file for review. There were 12 respondents, one of which also participated in a focus group held later in the study. Respondents represented clerical staff $(n=6)$, instructional staff $(n=3)$, and support staff $(\mathrm{n}=3)$ at each of the three grade levels. The majority of respondents worked at the middle school-level $(n=10)$, with one each at the elementary and high school-levels. The average number of years worked in their current positions was five years, although two respondents did not include this particular information. The lack of responses may be indicative of the lack of inclusion that classified staff may feel, as they are often not included in studies and their perspectives are not often sought after (Brown \& Stanton-Chapman, 2017; Carter et al., 2009; Downing et al., 2000; Feuerborn et al., 2018).

Although small in number, the survey respondents described their job responsibilities as greeting and enrolling students, administering medications, providing one-on-one assistance to students, filling in for teachers, and providing resources to remove barriers to learning. Seven of the 12 respondents stated that their responsibilities afforded them limited opportunities to interact with students when it came to behavior. There were a few that stepped in to deescalate tense situations and felt that enabled them to build relationships with students. One respondent (Family Resource and Youth Service 
Center -FRYSC) stated that they work with the mental health practitioner and provide a place for peer mediations and student “cool down” areas. Another respondent (a Lead Instructional Assistant) indicated that she dealt with behavior management while the teachers instructed the other students.

When asked about school-wide behavior expectations and PBIS specific training, the survey respondents identified multiple initiatives and varied levels of training and support. Two respondents mentioned an initiative called CHAMPS, a program used in the district several years prior but has not been a district initiative in over ten years. As mentioned in the review of literature, the CHAMPS initiative was a precursor to PBIS and has similar school-wide and classroom-based expectations that are clearly defined and modified to meet the needs of a particular school or classroom (Madigan et al., 2016). Two of the respondents mentioned training in Restorative Practices (RP) but gave little explanation that connected it to PBIS implementations.

Of the 12 survey respondents, seven affirmed that they had participated in PBIS training at some level during the past few years, while four indicated that they had never had any training. “I’ve only had partial training with PBIS for the first time this morning” (MS Clerk, survey), “I’ve only received training in school-wide” (MS- Library Clerk, survey), and “Yes, going over PBIS strategies” (Early Childhood IA, survey). Some additional feedback regarding PBIS training included details about what they thought was the purpose of PBIS, "From what I gather, it is calm, positive interactions, giving feedback and modeling correct behaviors" (MS Clerk, survey), and "I try to build students up and use positive and encouraging words and not focus on the negative” (MS Records Clerk, survey). 
Although limited, the responses from the surveys reveal a basic understanding of school-wide behavior expectations and some familiarity with programming currently used in their schools. Coding of focus group transcript data (undertaken after the surveys) revealed some similarities across data collection strategies and provided a means of triangulation. Inclusion of the survey results, although paltry in number, helps to synthesize the totality of the perspectives held by classified staff and corroborate data from focus groups.

\section{District Level Survey Documents}

The Fredrick County School District provides all employees an opportunity to complete an annual survey to provide information regarding school-wide culture and climate. District and school-level leaders use the results from the surveys to plan professional development, develop district-level and school-level initiatives, and assess leadership competencies at the school-level. The goal is that $100 \%$ of staff, both certified and classified, complete the surveys. For this study, I looked at the results for the five schools that participated to determine the level of participation amongst the classified staff on their Climate and Culture Survey and the measurement criteria for PBIS implementation.

The first survey I pulled from the available data was the Culture and Climate survey that looked at the overall function of a school. The survey data available at the time was the 2017-2018 data. The leadership in each of the participating schools had not changed during the interim time between the study and the completion of the survey. However, I was unable to determine if there had been changes in classified staff. Table 5 
compares certified and classified response rates of the 2017-2018 Culture and Climate Surveys for FCPS.

This particular study has four versions—one for certified staff, one for classified staff, one for parents, and one for students. This allows researchers and data technicians to look at specific role groups to determine needs for each school based on these categories. This also allows school leadership to take a deep look at the impact they have on subgroups within their building. Although the goal is $100 \%$ participation, there is limited incentive for completion for classified staff. Although district and school leadership mentioned survey feedback during their interviews, no classified participants mentioned participating in any surveys that were distributed.

Table 5. 2017-2018 Culture and Climate Survey

\begin{tabular}{lrr}
\hline \multicolumn{1}{c}{ School } & Certified Response Rate & Classified Response Rate \\
\hline Starfield ES & 71.4 & 22.6 \\
Fairview MS & 100.0 & 100.0 \\
Kingstown MS & 74.6 & 88.5 \\
Ashford Academy & 84.3 & 57.5 \\
Woodrow Wilson HS & 100.0 & 100.0 \\
FCSD Totals & 93.0 & 69.4 \\
\hline
\end{tabular}

The survey for classified staff consisted of thirteen questions in which the responses included the following options: strongly agree, agree, disagree, and strongly disagree. I reviewed the items on the survey that closely aligned to the features of PBIS that were pertinent to the study. These items included district and school-level leadership, feedback on job performance, the overall culture of the building, school safety concerns, and whether staff would send their own children to Fredrick County schools. The itemby-item agreement percentages are included in Table 6 and combined to show the levels 
at which classified staff agreed with the items that most aligned with the Self-Assessment Survey used to determine level of implementation of PBIS strategies.

Table 6. 2017-2018 CCS Agreement Percentages

\begin{tabular}{|l|r|r|r|r|r|r|}
\hline Survey Item Number & Starfield & Fairview & Kingstown & $\begin{array}{l}\text { Ashford } \\
\text { Academy }\end{array}$ & $\begin{array}{l}\text { Woodrow } \\
\text { Wilson }\end{array}$ & $\begin{array}{l}\text { Combined } \\
\text { Agreement }\end{array}$ \\
\hline $\begin{array}{l}1 \\
\text { District Leadership }\end{array}$ & 100.00 & 80.00 & 92.90 & 87.50 & 79.40 & 87.96 \\
\hline $\begin{array}{l}3 \\
\text { School Leadership }\end{array}$ & 100.00 & 87.50 & 88.90 & 87.50 & 85.70 & 89.92 \\
\hline $\begin{array}{l}6 \\
\text { Sending Own Children } \\
\text { to FCSD }\end{array}$ & 00.00 & 75.00 & 84.60 & 66.70 & 55.60 & 56.38 \\
\hline $\begin{array}{l}8 \\
\text { Feedback from } \\
\text { Supervisor }\end{array}$ & 50.00 & 93.3. & 88.90 & 75.00 & 94.10 & 80.26 \\
\hline $\begin{array}{l}10 \\
\text { Safe/Caring } \\
\text { Environment }\end{array}$ & 100.00 & 93.8. & 94.40 & 100.00 & 84.80 & 94.60 \\
\hline $\begin{array}{l}\text { 12 } \\
\text { Management of Safety } \\
\text { Concerns }\end{array}$ & 100.00 & 100.00 & 94.10 & 100.00 & 80.00 & 94.82 \\
\hline
\end{tabular}

From these survey data, it is evident that the classified staff members that responded to the survey had an above $80 \%$ agreement rate with most of the selected items. The highest agreement rates were with the overall care/concern that the schools provided (94.60) and the management of safety concerns (94.82). These two items are essential to fostering the core components of PBIS in which school-wide systems are enacted in order to meet the needs of the students and staff. This supportive learning environment is the focus of the district's plan and therefore it is important to have agreement about how to care and support students. Additionally, the classified staff felt that support and leadership at both the district and school-levels were satisfactory to the majority of respondents. 
The purpose of using these survey data is to shed light on how classified staff rate their schools on the CCS. This is the only measure provided by the district that specifically separates certified and classified responses to issues of school climate. The low response rate is indicative of the disengagement that classified staff may feel in overall decision making and planning. The response rates were low in three of the five schools participating in the study. This finding in consistent with the types of responses participants gave during focus group discussions. During discussions the majority of the participants expressed that their schools provided care and support to students and handled disruptions in a timely manner, and the two questions in the short survey showed over $90 \%$ agreement. Additionally, many of the participants did not voice concerns about district or school leadership, as evidenced by survey responses that show over $80 \%$ agreement.

Surprisingly, the responses to sending their own children to FCSD was low (56.38) considering that ratings on overall climate and handling of safety concerns was high and the focus group participants did not express dire concerns that would indicate a dissatisfaction that would hinder their decisions to send their children to the school district for which they worked. It is likely that other factors affect staff decisions regarding their children's placement.

The second survey reviewed for this study was the Self-Assessment Survey (SAS) that is specific to PBIS implementation. The district administers the survey to all certified and classified staff and there is no way to disaggregate results by role groups. Administered between October and November of each school year, the district uses the survey and its results to develop PBIS action plans for that school year. The district 
urges PBIS planning teams to use the results from the SAS as a guide for their monthly meetings and to ensure feedback to the entire staff.

As with the culture and climate survey, the district's goal for the SAS is to have a $100 \%$ participation rate from staff members. The SAS consists of four sections: schoolwide systems, non-classroom settings, classroom settings, and individual students. Within each of these sections there are between nine and eighteen questions that respondents must rank as in-place, partially in-place, or not in-place, followed by marking as a high, medium, or low improvement priority. The district deems any responses below $50 \%$ as areas of improvement for PBIS teams to address.

I pulled the survey data for the current school year (2019-2020) for the five participating schools. I reviewed items on the survey that most directly aligned with the guiding questions for this study. In Appendix F, I provide an overview of the responses from each of the participating schools. The five schools that participated in the study had varying degrees of PBIS implementation, according to district data provided by Sarah during her interview. October 2019 discipline data (See Table 3) showed that office disciplinary referrals (ODR) increased with each grade band, even though the elementary and middle schools had been using PBIS strategies for multiple years. Review of the SAS shed light on why variation in ODR rates exist.

Identification as "fully implemented" by the district requires schools score at or above $80 \%$ in each of the system categories on the SAS. The overview in Appendix F provides the percentages at each school on their most recent survey. Table 7 provides the overall “in-place” percentages of combined responses. Of the 19 items included in the summary, ten of the indicators were below $50 \%$, and no categories reached the $80 \%$ 
threshold that indicates full implementation of PBIS within the 'fully in-place' status.

The data provided by the district indicated that three of the five schools (Starfield, Fairview, and Kingstown) are at high levels of implementation because they combined "fully in-place” and "partially in-place” to show that schools are making progress toward implementation.

In all, all participating school had four survey items had low percentages. These included “consequences for problem behavior is defined clearly” (23.4\%), "limited options for classroom instruction to continue during behavior problems” (35.00), "full staff involvement, both directly and indirectly, with interventions” (39.40), and "budgeting for specific PBIS components of teaching students, on-going rewards, and staff training” (37.20). Additionally, when accounting for the 'partially in-place’ category, three of the four categories still fell short of the $80 \%$ threshold to be considered successfully implemented according to the SAS expectations. The three categories that still fell below $80 \%$ were consequences for problem behavior defined (73.00), budgeting priorities (79.20), and full staff involvement (75.00).

Table 7. 2019 SAS Overall Percentages for Featured Items

\begin{tabular}{|c|c|c|c|}
\hline Feature Item & $\begin{array}{c}\text { Overall In- } \\
\text { Place \% }\end{array}$ & Feature Item & $\begin{array}{c}\text { Overall In- } \\
\text { Place \% }\end{array}$ \\
\hline $\begin{array}{l}\text { Positive/clear student } \\
\text { expectations defined }\end{array}$ & 73.60 & $\begin{array}{l}\text { Behaviors taught } \\
\text { directly }\end{array}$ & 52.80 \\
\hline Problem behaviors defined & 44.20 & Clear consequences & 28.40 \\
\hline $\begin{array}{l}\text { Continuous instruction } \\
\text { continues }\end{array}$ & 35.00 & Procedures in place & 66.60 \\
\hline PBIS team exists & 61.20 & $\begin{array}{l}\text { School } \\
\text { administrators are } \\
\text { active }\end{array}$ & 74.40 \\
\hline
\end{tabular}




\begin{tabular}{|l|l|l|l|}
\hline $\begin{array}{l}\text { Behavior patterns used to } \\
\text { inform decisions }\end{array}$ & 45.40 & $\begin{array}{l}\text { Budget exists for } \\
\text { PBIS }\end{array}$ & 37.20 \\
\hline Full staff involvement & 39.40 & $\begin{array}{l}\text { Ongoing district } \\
\text { support }\end{array}$ & 55.40 \\
\hline $\begin{array}{l}\text { School-wide expectations } \\
\text { for non-classroom setting }\end{array}$ & 59.40 & $\begin{array}{l}\text { Behaviors taught } \\
\text { for non-classroom } \\
\text { settings }\end{array}$ & 42.20 \\
\hline $\begin{array}{l}\text { Opportunities for } \\
\text { development/improvement } \\
\text { of skills }\end{array}$ & 42.80 & $\begin{array}{l}\text { All staff } \\
\text { directly/indirectly } \\
\text { involved in non- } \\
\text { classroom setting s }\end{array}$ & 50.80 \\
\hline $\begin{array}{l}\text { Teachers have access for } \\
\text { assistance* }\end{array}$ & 57.60 & $\begin{array}{l}\text { Prompt response } \\
\text { from behavior team }\end{array}$ & 44.00 \\
\hline $\begin{array}{l}\text { Feedback provided to } \\
\text { behavior team }\end{array}$ & 49.80 & & \\
\hline
\end{tabular}

*Denotes the question only addressed certified staff access

The SAS data revealed that none of the schools in the study had "In Place" indicators that were above $80 \%$. However, when accounting for "in-place” and "partially in-place”, four of the five schools has $80 \%$ in the majority of categories included on the survey. In particular, both high schools had higher levels of ODR than the elementary and middle schools, and both high schools showed a lower level of fully or partially inplace PBIS implementation strategies. Ashford Academy had 5 indicators above $80 \%$ inplace and 28 indicators that showed partial in-place. In contrast, Woodrow Wilson had one indicator above $80 \%$ and 32 indicators that showed partial implementation. When looking at the average number of ODR for each school, these align in practice, as Woodrow Wilson has the highest number of ODR per day on average.

The SAS results do not distinguish between certified and classified responses and only look at the whole staff completion rate. What the SAS does show is that school staff, in general, have mixed views about how impactful PBIS is in their particular building holistically. This makes the focus group sessions valuable in providing a direct 
perspective of the work that classified staff take on when assisting with behavior interventions that fall under the PBIS umbrella.

PBIS is a complex initiative that takes time to implement fully within one school, yet, the FCSD moved to full-scale implementation as a normal function of the entire organization with limited whole scale training for all employees. These data are useful for decision making within the school district. The next section will look at how all three data points (on-line survey, focus group sessions, and district-level surveys) work in conjunction with each other as part of the normalization of a complex organization system.

\section{Perspectives of Inclusion: Emerging Themes}

When Fredrick County School District made the decision that all its schools would implement PBIS system-wide at all grade bands during the 2019-2020 school year, it was an expansion of an initiative already used in many of the district's schools. However, this was the first time that the district made it a requirement for all schools. In order to achieve that goal, FCSD issued mandatory initial training for schools based on prior year's implementation levels in order to provide appropriate levels of support. District leadership made determinations about school-levels, and each school was required to send a core team to training in the spring of 2019, in order to prepare for the opening of school during the following school year.

With good intentions and a plan of action, district leadership began training school-level teams and provided additional professional learning opportunities throughout the summer of 2019. What was revealed during this study were varied 
perspectives of what this looked like for different role groups that would be required to implement PBIS strategies. The remainder of this section will look at the varied perspectives and emerging themes regarding the inclusion of classified staff in the successful implementation of PBIS.

\section{Relationship Building}

When coding for themes, both leadership and classified staff identified some commonalities. The first common theme to emerge from this analysis revolved around relationships. Although the word "relationship" appeared in the interviews and focus groups minimally, related terms and descriptions interwove throughout the discussions. Leadership interviews spoke of "the energy, verbiage, and mindset that comes naturally [to classified staff]" (Gwen), and "they're actually [a] really big peg that helps our system” (Candace). Kristen mentioned that her classified staff have "a very different relationship with kids" and "they are also those people that some of the students open up to” (Candace).

Leadership also spoke about the role many classified staff members have with students that supports the relationship theme. They described situations where classified staff were there to deescalate situations, conduct home visits, work with students in nonclassroom settings like the cafeteria and provide overall safety measures for all students. For many of these leaders, their description of the work that classified staff members did on a daily basis are an intervention component of PBIS. For example, Frances recalled that “most of their work, even though it's not outright about PBIS, oftentimes they're reinforcing the classroom routines and trying to assist”. The relationship building takes 
place in these situations of assistance when "they (CS) take the time out to get to know them [students] more on their own level” (Candace).

Classified staff shared this same view of relationships in the focus groups. Again, the word "relationship" was not used often (only four mentions from transcripts overall). Relationship descriptors were described by all levels with terms like mentor, father figure, uncle, someone to hug and listen to them, and personality were used to describe their interactions with students. Throughout the focus group discussions, the staff spoke about their level of interventions with students as the main part of their job (especially at the middle and high school-levels) and viewed themselves as being a support system for students in need and a safe place for students to express themselves.

Classified participants mentioned that they felt that listening to students was an important part of their work. Comments like, "giving them a hug” (Debbie, ES) “someone to listen to" (Leslie, MS), "father/uncle figure” (Steve, HS), and “mentor” (John, MS) were mentioned at several points throughout the three sessions. Although most of the participants did not talk specifically about PBIS as an initiative, they were all able to talk about their relationships with students and the interactions that they felt helped to calm and support students.

Relationship building looked similar at each grade band in which these classified staff members often stepped in to provide safe spaces for students to express themselves. Many of the participants shared personal connections that they made with students when emotions ran high. Connie (Starfield ES) recalled a student who was being disrespectful to his teacher in the cafeteria, "So I talk to him, you know... And how about if you do this all week and Friday, if she says you were good, I'll have something for you. [On Friday] 
she said she had not had a problem with him since.” Robert (Fairview MS) described how he works with students stating, "So we might have to get a kid out [of class] and bring them to the office. They get over there, get to crying and go through whatever. After they finish all of that, then we can start a conversation. We'll start a dialogue.” Ronnie (Woodrow Wilson HS) also spoke about the connections with students, "See, because we had those kinds of relationships with kids, they're going to let us know... they're going to tell us”. All of these relationship examples highlight the view that classified staff in these focus groups hold concerning their interactions with students.

The other part of relationships discussed were the physical spaces that classified staff provided for students to deescalate and express themselves when problems occurred. The middle and high school participants shared examples of how they felt their relationships with students were on a different level than the relationships that students had with teachers. Robert (Fairview MS) stated that, "lots of tears have been shed on this couch” when referring to upset students who were brought to his office. He provided an intervention for students prior to any contact with school administration for disciplinary actions. His work with John to mediate situations to help students regroup and get back to class quickly was more of a mentorship to them. This type of intervention with students who face social challenges aligns with the tiered support system of PBIS (Simonson \& Myers, 2015).

The focus group participants also talked about their work to give teachers a break from students and students a break from teachers. John (Fairview MS) stated that teachers often credit his team for their interventions when students are being difficult in class, "We'll take them out, not so much for their [the student] sake...it’s more like, 
okay, ma’am, sir, needs a break”. Lawrence and Ronnie (Woodrow Wilson HS) describe the interactions with teachers and students in a different manner. Both described students who do not want to be in classrooms and students who can be "hell raisers" just to get out [of the rooms]. Some teachers willingly hand out restroom passes just because "the teacher gets a little break” (Ronnie). For Lawrence and Ronnie, they continue to work with students to try to get them back in the classrooms so they can be "college and career ready”. At the elementary level, there were no mentions of student being removed from classes or giving them breaks when students were emotionally escalated.

\section{Explicit Training}

The second theme that emerged from the analysis of data was the lack of explicit understanding of PBIS, even though the descriptions they provided of their daily work responsibilities support the core vision of the initiative. Simonsen and Myers (2015) describe PBIS as an evidence-based prevention framework that provides a continuum of support for all students. Without using the acronym of PBIS, participants described that they gave students space and time to deescalate when they were upset, provided interventions to students in order to avoid additional punitive measures with administrators, and provided opportunities for students to check-in with them on a consistent basis.

At all three grade bands, the classified staff talked about strategies they have developed within their own collegial team to help students. Although they talked at great length about their work in the cafeterias, hallways, and classrooms, they did not talk specifically about the direct connection of their work to PBIS expectations. As John (Fairview MS) put it, "It's just what we do”. All of the focus group participants were 
able to articulate the need for students to have positive reinforcements and consistency with school rules and expectations, even though they were not involved in the development of such plans at the school-level.

The first question asked during each of the focus group session was "What are your understandings of the goals and expectations of Positive Behavior Interventions and Supports?” In all three focus groups, this first question met with hesitation on the part of the participants. Watching the replays of the sessions revealed participants glancing at one another prior to the first person speaking. I interpreted this as trying to determine who would take the risk to be the first person willing to begin the conversation. Once the first person spoke up, other participants quickly followed. With this particular question, the first person to speak in each of the grade bands simply restated part of the question, “positive behavior interventions” (Diane, transcript), "positive reinforcements” (Leslie, transcript), and “conferencing, positive reinforcement” (Keith, transcript).

These first responses opened the door for the other participants to speak up. What it revealed was the lack of a clear perspective on PBIS as an initiative. Robert (Fairview MS) has worked at his school for 14 years, and his role places him in contact with students categorized as Tier 2 or Tier 3 according to the PBIS framework. Although his principal stated that Robert was actively involved in the process, Robert revealed he did not have a clear understanding of how to implement the framework. Other coworkers offered responses confirming this lack of understanding.

Participants from elementary and high school focus groups offered similar responses. At Starfield, the participants who had been at the school for over two years could only recall their participation in a two-day training with limited follow-up 
opportunities for classified staff. The newest member of the Starfield staff, Diane, had not received any training and only picked up ideas based on overhearing others talk about PBIS. At Woodrow Wilson, the security team had no training in PBIS methods or strategies but complied with a hands-off physical approach as directed by their assistant principal. However, all of the Woodrow Wilson participants actualized what PBIS stood for in terms of how they interacted with students as a "natural" part of their overall personalities.

According to Rodriguez, Loman, and Borgmeier (2016), many schools are implementing interventions for students without receiving adequate training aligned with PBIS. This was evident during the focus group discussion in this study. In all, only five of the 15 participants had been involved in explicit PBIS training within the past two years, and none of the 15 participant were actively involved in the PBIS teams at their schools. At the middle and high school-levels, the lack of training has the potential to influence their success when trying to deescalate students removed from the classroom setting when staff do not have the background knowledge regarding why specific strategies are more effective than others are. At these particular schools, the participants worked as cohesive units based on their personalities and willingness to work together. Coaching and training staff is a significant predictor of PBIS being successful in a school (McIntosh et al., 2016) and this lack of explicit training could lead to the breakdown of cohesion should members of the current teams that participated in this study change jobs.

Although the participants expressed predominately positive views of their daily work, they showed a lack of in-depth understanding of PBIS implementation fidelity. In each of these groups, the team developed their own levels of engagement without 
significant guidance from school or district leadership. The only mention of school or district leadership in the elementary group was their discussion of training in years past. At the middle and high school-levels, they only mentioned school or district leadership when it concerned the processes their team used to intervene on the behalf of students prior to involving administration in disciplinary actions.

The disconnect between PBIS as a mandated district initiative and the day to day job responsibilities of classified staff was made clear during focus group discussions. While district and school leadership were well versed in the components of PBIS and the procedures to establish school-wide teams, classified participants were vaguely aware of the structures that leadership referred to during their individual interviews. In the next section, I will discuss the findings as they relate to normalizing PBIS within the participating schools.

All participants in the study identified training as an area of growth for the district and for individual schools. Although systems are in place for PBIS training and support, there is not a coherent plan for ensuring that all stakeholders get the same level of professional development. Each participant group (district leadership, school leadership, and classified staff) agreed in the need for more training, but there was no consistent plan for implementation.

At the district-level, Sarah and Yvonne had clear plans for training schools and offered PBIS sessions throughout the school year. They also ensured that all schools completed the SAS and the TFI annually and encouraged schools to use that information to develop action plans. They made recommendations for team membership and provided a resource teacher to each school (although these resource teachers served 
multiple schools). The intention of district leadership was to provide materials, training, and support to all schools for PBIS implementation under the umbrella of Multi-tiered Systems of Support (MTSS).

Expectations and the reality are two different things. School leadership described whole staff training that included certified and classified staff at the beginning of the year to "revisit what our [PBIS] goals are and what we're trying to do" (Frances). However, after these initial reviews, classified staff were no longer considered a priority for inclusion in school-based training during the remainder of the school year. This was not something that school leaders did with malice, but four of the five school leaders did not have the budgetary focus to pay classified staff to stay for staff meetings after school when PBIS updates would normally be planned for certified staff. As Candace put it, “To be quite honest, they're not considered high on the priority list. They're not considered a priority. They're actually a second thought—second or third thought”.

Classified staff who participated in focus groups triangulated this. At the elementary level, all of the participants had knowledge of PBIS, and 4 of the 5 participants had participated in one training, two years prior to the focus group. The fifth member of that group had received no training and had only picked up what she knew through communication with her lead teacher. At the middle school-level, only one had participated in a PBIS training in previous years, and the others had no specific training and did not mention anything from the beginning of the year with the whole staff. At the high school-level, they had the least knowledge of PBIS and participated in no specific training sessions. 
Classified staff did not mention attending any staff meetings (as this is not part of their scheduled workday) and did not participate as members of the PBIS team for their particular school. Although they lacked specific training, all of the participants showed a level of basic understanding and support for PBIS, even when they did not know terminology or strategies. The potential inclusion of classified staff in the training of PBIS components and techniques is a natural fit for the work that they do and should be taken seriously by district and school-level leadership when planning and designing training for staff members.

When discussing their understanding of PBIS, the focus group participants spoke about informal verbal communication. School leaders interviewed for this study also indicated that classified staff usually worked with the teachers when learning about PBIS. Neither school leadership nor classified staff participants mentioned formal training or documentation like the SAS or TFI results to develop training modules or inform school PBIS plans. District and school leaders did not indicate any plans for improving training for classified staff in the future, only that it was something that they needed to consider.

Although focus group participants relied on limited information regarding PBIS, this did not stop them from implementing strategies that they felt worked well with students. All participants held the work that they did in a positive regard and were committed to continuing current practices, even with reduced training. The fact that classified staff show support for an initiative in which they have partial inclusion should be encouraging for district and school leaders who are planning future professional development opportunities. 
It is important to note that building level leaders recommended classified staff participants for this case study based on their particular job responsibilities. Although there is nothing to suggest that administrators recommended classified staff participants in order to give a positive spin on PBIS implementation, this fact bears consideration when analyzing their responses. The classified staff members' unique roles with support and intervention gives them first-hand knowledge of real situations of PBIS strategies utilized in reactionary situations. The elementary group was the only group that worked in isolation from each other and did not have a system that they followed as a unit.

This does not mean that their perspective on PBIS inclusion is not reliable or valid. The work that they do is representative of the work that classified staff throughout FCSD take part in on a daily basis. Their levels of participation in staff meetings and professional development is consistent with district-level accounts that Sarah and Yvonne spoke about in their interviews. Additionally, only three members of the focus groups talked about their inclusion in PBIS and training in other schools they worked in previously that are consistent with their current perspectives.

Two schools, Kingstown Middle and Ashford Academy (high school), did not participate in focus groups. However, the leadership interviews at these schools corroborates the lack of inclusion in PBIS training for their classified staff members. At Kingstown, Candace called this the 'white elephant' in the room when asked about how classified staff participate in meetings and trainings. She stated that there was a lack of resources in the budget and an assumption that classified staff will just follow through based on what they see as the norms of the school. 
The principal at Ashford Academy said she felt a need to train all of the staff, and she was willing to pay classified staff or train them during the school day, but she offered no specific details on when that would occur. She also stated that she was not sure if classified staff were using PBIS like the other staff, and there was no mention of how she would determine overall usage. These two interviews shed light on the overall lack of consideration that FCSD has made to be intentional about including classified staff in PBIS training and support. Indeed, none of the schools that participated had a solid plan for their classified staff to develop their skills or deepen their understanding of PBIS. Additionally, there was not a concrete plan at the district-level to mandate the inclusion of all classified staff in training and only made recommendations to individual schools to consider. Leadership views of classified staff were presumptive of their ability to just pick up PBIS through observations and informal conversations.

The classified staff were well aware that they had limited information on PBIS but did not find that it hindered their jobs in any way. They were willing to participate in training if such opportunities availed themselves but did not suggest feeling left out or unappreciated. At all three levels, they indicated that they felt their work naturally fit with PBIS overall and expressed an appreciation for the positive reinforcement that their schools focused on to help reduce severe disciplinary consequences that had been used in the past.

These findings are encouraging for the advancement of this complex organizational initiative. FCSD has a district leadership team that has made an effort to provide all schools with an overview of PBIS and tools to implement the program. School leaders are putting plans in place to ensure that PBIS utilization throughout their 
buildings. Classified staff are in the action with students and are willing to learn about PBIS strategies that support their work.

The challenge now is to develop a systemic plan to ensure that classified staff are able to participate in an equal amount of training as their certified counterparts. Mandated programs need to have mandated participation if they are to be successful. This means that the inclusion of classified staff in all aspects of PBIS training is essential to the success of the program. Members of this role group have a stake in FCSD, and all participants in the study expressed a desire to help support their schools, their supervisors, the teachers, and the students. Including them in district-level and schoollevel PBIS training will only enhance their work and benefit students.

\section{Theory in Action-Normalization Process Theory}

NPT allows researchers and organizational leaders to look at systems to ensure that they are utilizing the necessary steps to have a successful implementation process for new initiatives. Large organizations like FCSD can ensure that all stakeholders have the opportunity to make sense of a program (coherence), be a participant in the action (collective action), utilize their understanding to buy-in to the program (cognitive participation), and improve their job performance (reflexive monitoring) if measures are put into place to train and include all stakeholders throughout the implementation. While PBIS is not a completely new initiative in FCSD, this is the first year that all grade bands were required to implement the initiative, and some groups were not engaged in the process. 


\section{Coherence}

During the interviews and focus groups it became clear that the idea of coherence was not aligned between leadership and classified staff. During all of the individual interviews, leadership described beginning of year training sessions in which all staff participated, district-level training with different classified role groups (instructional and non-instructional staff), utilization of survey data for decision making, and a sense that classified staff would "pick-up” the information through conversations with teachers and other colleagues. The leadership participants also acknowledged not prioritizing the inclusion of classified staff members in an on-going training model

The focus group participants expressed levels of understanding that was separate from specific training participation. They made sense of PBIS through second-hand accounts and overheard conversations from teachers and other staff (as described by leadership). Their depth of knowledge concerning PBIS was to keep suspensions down (middle and high school-levels), limit physical contact like restraints (high school), and to help deescalate situations when students were upset (all three levels). However, those who participated in the focus groups expressed a natural connection to the core concepts of PBIS when it came to being positive and giving positive reinforcement.

The focus group participants and the classified staff that completed the study survey expressed limited training in specific PBIS school-based strategies. This did not stop them, however, from engaging with students in a supportive role and creating a safe space for students to deescalate before reintroduction to the classroom setting. Their understanding of PBIS was surface level in terminology and specificity but deeper when describing their connections with students and their roles within their buildings. 


\section{Cognitive Participation}

When it came to cognitive participation, all focus group participants described their daily responsibilities and spoke clearly about the role they played in the day-to-day interactions with students. Most saw themselves as behavior interventionists that stepped in to support certified teachers when difficulties with students arose. They perceived their work with colleagues as a second tier of support, upholding the behavioral expectations that school administration had put into place. All expressed a willingness to do whatever is necessary, even if they lacked some of the core strategic information.

As a collective group, the classified staff that completed the online survey and those that participated in the focus groups expressed their interactions with colleagues when it came to discipline or PBIS expectations as compartmentalized within their role group. In the survey several responded that administration was included as back-up if a student needed extra behavioral interventions, seeking the assistance of colleagues when they didn't know how to follow-up, and waiting for security or administration to step into intense situations. These responses showed a variance of either withdrawing from disciplinary interactions with students to allow "others" to handle difficult situations or jumping in as first responders to deescalate.

The relational work of cognitive participation was not evident when cross analyzing leadership interviews, on-line survey responses, and focus group discussions. Each group felt that others had more information regarding PBIS and there was no clear path to ensuring that all staff would receive adequate training in the future. Groups worked in pods of knowledge within their role group with classified staff receiving the 
least amount of overt support and training to ensure that they were able to implement PBIS completely and effectively.

\section{Collective Action}

Collective action is the "operational work that people do to enact a set of practices” (Lloyd et al., 2013, p. 4). At both the middle and high school-level, the group consisted of a team that worked together in a particular role within their building. Their views of their work were a collective effort. They described themselves as developing their own system and hierarchy where communication between members of their "team" received priority over working with administration directly. Both of these groups had limited guidance from their administrators but did feel that the school leadership respected their opinions and decisions about students. They expressed respect for each other and acknowledged their personal strengths and weaknesses as team members.

At the elementary level, the participants did not work as a group and had specific roles that included non-instructional and instructional responsibilities. Therefore, they characterized their work responsibilities as siloed when implementing PBIS components as opposed to collective team action. Although they displayed mutual levels of respect for each other, they did not have the same dynamic as the middle and high school teams tasked with one specific component (behavior management). Their view was holistic, and they could operate as a "spectator" in some of their roles (bookkeeper and secretary) or be directly engaged, like the cafeteria monitor and two instructional assistants.

For leadership, the concept of collective action was a top-down model in which the district provided training, resources, and staff to assists school leaders. These school 
leaders had some autonomy to ensure that PBIS implementation was specific to the needs of their schools and their students while aligning with district planning documents. Beyond that, the level of responsibility regarding the actions taken at each school consisted of the two fidelity measures (SAS and TFI) and feedback that resource teachers provided to school-level PBIS teams, when invited to attend school-level meetings. School leaders outlined the actions that they took to involve certified staff within the PBIS implementation process but had minimal action steps for classified staff.

\section{Reflexive Monitoring}

The final component of NPT is reflexive monitoring, or the ability to get feedback to assess and improve job performance. This area was the most lacking component in both interviews and focus group sessions. Neither leadership nor classified staff acknowledged in-depth feedback loops other than end of year evaluation or situational feedback after a specific incident. Additionally, the SAS had low percentages when asked about whole staff involvement in PBIS initiatives.

Lack of in-depth training for classified staff and lack of direct feedback on a consistent basis does not allow for continuous growth and improvement. Much of this links to the lack of budgetary priorities for classified training that school leaders discussed during interviews. Only one principal (Kristen, Ashford Academy), identified a budgetary priority for her classified staff and that was for Safe Crisis Management (SCM) training each year. When asked about their needs during the focus group sessions, all groups expressed interest in participating in specific PBIS training should it be provided at either the district or school level. 
With time and training, a complex initiative like PBIS may eventually normalize within a large organization system like FCSD where all stakeholder within the system are included in the process. Evidence from the interviews and focus groups show a level of basic understanding that could propel FCSD to success. In contrast to each other, all participants were able to show coherence of the PBIS initiative as it applies to the district. However, all participants acknowledged a lack of reflexive monitoring. District and school leadership expressed that they needed to make more considerations for their classified staff when planning professional development and training. Classified staff did not express a need for feedback but said that it was minimal. Without a plan for consistent feedback opportunities, classified staff will continue to rely on second-hand information overheard from certified staff members. This does not bring them into the action of PBIS needed to utilize their abilities fully.

District leadership acknowledged the need for PBIS training for classified staff, but did not have a concreate plan to ensure that there was enough information provided at the district-level to ensure that all stakeholders were included consistently. At the schoollevel, leadership said that classified staff were part of their PBIS team, but this was not in alignment with the responses from any of the classified staff that participated with the online survey or the focus groups. Tables 8 and 9 show the breakdown of the NPT's core tenets and codes embedded in each category through analysis of participant interview and focus group transcripts. 
Table 8. Coding and Themes among Leadership Participants

\begin{tabular}{|c|c|c|c|c|c|c|c|c|}
\hline \multirow{2}{*}{ NPT Tenet } & \multirow[b]{2}{*}{ Theme } & \multicolumn{2}{|c|}{ District-Level } & \multicolumn{5}{|c|}{ School-Level } \\
\hline & & Sarah & Yvonne & Candace & Beth & Gwen & Kristen & Frances \\
\hline \multirow{3}{*}{ Coherence } & $\begin{array}{l}\text { Shared } \\
\text { Understanding }\end{array}$ & $\mathrm{X}$ & $\mathrm{X}$ & & & & & $\mathrm{X}$ \\
\hline & $\begin{array}{l}\text { Roles and } \\
\text { Responsibilities }\end{array}$ & $\mathrm{X}$ & $\mathrm{X}$ & $\mathrm{X}$ & $\mathrm{X}$ & $\mathrm{X}$ & & $\mathrm{X}$ \\
\hline & $\begin{array}{l}\text { Value and } \\
\text { Beliefs }\end{array}$ & $\mathrm{X}$ & $\mathrm{X}$ & $\mathrm{X}$ & $\mathrm{X}$ & & $\mathrm{X}$ & $\mathrm{X}$ \\
\hline \multirow{3}{*}{$\begin{array}{l}\text { Cognitive } \\
\text { Participation }\end{array}$} & $\begin{array}{l}\text { Key Leaders } \\
\text { Identified }\end{array}$ & $\mathrm{X}$ & $\mathrm{X}$ & $\mathrm{X}$ & $\mathrm{X}$ & & $\mathrm{X}$ & \\
\hline & $\begin{array}{l}\text { Demonstration } \\
\text { of "buy-in" }\end{array}$ & $\mathrm{X}$ & $\mathrm{X}$ & & $\mathrm{X}$ & & & $\mathrm{X}$ \\
\hline & $\begin{array}{l}\text { Involvement in } \\
\text { Intervention }\end{array}$ & $\mathrm{X}$ & $\mathrm{X}$ & $\mathrm{X}$ & $\mathrm{X}$ & $\mathrm{X}$ & $\mathrm{X}$ & $\mathrm{X}$ \\
\hline \multirow{3}{*}{$\begin{array}{l}\text { Collective } \\
\text { Action }\end{array}$} & $\begin{array}{l}\text { Interactions } \\
\text { between sub- } \\
\text { groups }\end{array}$ & $\mathrm{X}$ & $\mathrm{X}$ & $\mathrm{X}$ & $\mathrm{X}$ & & $X$ & $\mathrm{X}$ \\
\hline & $\begin{array}{l}\text { Knowledge } \\
\text { base }\end{array}$ & $\mathrm{X}$ & $\mathrm{X}$ & $\mathrm{X}$ & $\mathrm{X}$ & $\mathrm{X}$ & $\mathrm{X}$ & $\mathrm{X}$ \\
\hline & $\begin{array}{l}\text { Integration } \\
\text { within daily } \\
\text { work }\end{array}$ & & & & $\mathrm{X}$ & $\mathrm{X}$ & & $\mathrm{X}$ \\
\hline \multirow{4}{*}{$\begin{array}{l}\text { Reflexive } \\
\text { Monitoring }\end{array}$} & $\begin{array}{l}\text { Individual } \\
\text { Effectiveness }\end{array}$ & & & & & & & \\
\hline & $\begin{array}{l}\text { Performance } \\
\text { Evaluation }\end{array}$ & $\mathrm{X}$ & $\mathrm{X}$ & $\mathrm{X}$ & $\mathrm{X}$ & & $\mathrm{X}$ & $\mathrm{X}$ \\
\hline & $\begin{array}{l}\text { Application of } \\
\text { Strategies }\end{array}$ & & $\mathrm{X}$ & $\mathrm{X}$ & $\mathrm{X}$ & & $\mathrm{X}$ & $\mathrm{X}$ \\
\hline & $\begin{array}{l}\text { Intentional } \\
\text { Feedback }\end{array}$ & $\mathrm{X}$ & $\mathrm{X}$ & & & & & \\
\hline
\end{tabular}


Table 9. Coding and Themes among Classified Staff Participants

\begin{tabular}{|c|c|c|c|c|c|c|c|c|c|c|c|c|c|c|c|}
\hline & & \multicolumn{5}{|c|}{ Elementary } & \multicolumn{4}{|c|}{ Middle } & \multicolumn{5}{|c|}{ High } \\
\hline NPT Tenet & Theme & Rhonda & Susanne & Debbie & Connie & Diane & John & Deborah & Leslie & Robert & Ronnie & Micah & Lawrence & Rodney & Steve \\
\hline \multirow{3}{*}{ Coherence } & $\begin{array}{l}\text { Shared } \\
\text { Understanding }\end{array}$ & & & $\mathrm{X}$ & $\mathrm{X}$ & $\mathrm{X}$ & $\mathrm{X}$ & & & $\mathrm{X}$ & & & & & \\
\hline & $\begin{array}{l}\text { Roles and } \\
\text { Responsibilities }\end{array}$ & $\mathrm{X}$ & $\mathrm{X}$ & & $\mathrm{X}$ & $\mathrm{X}$ & $\mathrm{X}$ & $\mathrm{X}$ & & $\mathrm{X}$ & $\mathrm{X}$ & $\mathrm{X}$ & $\mathrm{X}$ & $\mathrm{X}$ & $\mathrm{X}$ \\
\hline & $\begin{array}{l}\text { Value and } \\
\text { Beliefs }\end{array}$ & & & $\mathrm{X}$ & & $\mathrm{X}$ & $\mathrm{X}$ & & & $\mathrm{X}$ & $\mathrm{X}$ & $\mathrm{X}$ & $\mathrm{X}$ & $\mathrm{X}$ & $\mathrm{X}$ \\
\hline \multirow{3}{*}{$\begin{array}{l}\text { Cognitive } \\
\text { Participation }\end{array}$} & $\begin{array}{l}\text { Key Leaders } \\
\text { Identified }\end{array}$ & $\mathrm{X}$ & $\mathrm{X}$ & $\mathrm{X}$ & $\mathrm{X}$ & $\mathrm{X}$ & & $\mathrm{X}$ & $\mathrm{X}$ & $\mathrm{X}$ & & & & & \\
\hline & $\begin{array}{l}\text { Demonstration } \\
\text { of "buy-in" }\end{array}$ & & $\mathrm{X}$ & $\mathrm{X}$ & $\mathrm{X}$ & $\mathrm{X}$ & $\mathrm{X}$ & $\mathrm{X}$ & $\mathrm{X}$ & $\mathrm{X}$ & $\mathrm{X}$ & $\mathrm{X}$ & $\mathrm{X}$ & $\mathrm{X}$ & $\mathrm{X}$ \\
\hline & $\begin{array}{l}\text { Involvement in } \\
\text { Intervention }\end{array}$ & & & $\mathrm{X}$ & $\mathrm{X}$ & $\mathrm{X}$ & $\mathrm{X}$ & $\mathrm{X}$ & $\mathrm{X}$ & $\mathrm{X}$ & $\mathrm{X}$ & $\mathrm{X}$ & $\mathrm{X}$ & $\mathrm{X}$ & $\mathrm{X}$ \\
\hline \multirow{3}{*}{$\begin{array}{l}\text { Collective } \\
\text { Action }\end{array}$} & $\begin{array}{l}\text { Interactions } \\
\text { between sub- } \\
\text { groups }\end{array}$ & & & & & $\mathrm{X}$ & $\mathrm{X}$ & $\mathrm{X}$ & $\mathrm{X}$ & $\mathrm{X}$ & $\mathrm{X}$ & $\mathrm{X}$ & $\mathrm{X}$ & $\mathrm{X}$ & $\mathrm{X}$ \\
\hline & $\begin{array}{l}\text { Knowledge } \\
\text { base }\end{array}$ & & $\mathrm{X}$ & $\mathrm{X}$ & $\mathrm{X}$ & $\mathrm{X}$ & $\mathrm{X}$ & $X$ & $\mathrm{X}$ & $\mathrm{X}$ & & & & & \\
\hline & $\begin{array}{l}\text { Integration } \\
\text { within daily } \\
\text { work }\end{array}$ & & & $\mathrm{X}$ & $\mathrm{X}$ & $\mathrm{X}$ & $\mathrm{X}$ & $\mathrm{X}$ & $\mathrm{X}$ & $\mathrm{X}$ & $\mathrm{X}$ & $\mathrm{X}$ & $\mathrm{X}$ & $\mathrm{X}$ & $\mathrm{X}$ \\
\hline \multirow{4}{*}{$\begin{array}{l}\text { Reflexive } \\
\text { Monitoring }\end{array}$} & $\begin{array}{l}\text { Individual } \\
\text { Effectiveness }\end{array}$ & & $\mathrm{X}$ & $\mathrm{X}$ & $\mathrm{X}$ & $\mathrm{X}$ & $\mathrm{X}$ & $\mathrm{X}$ & $\mathrm{X}$ & $\mathrm{X}$ & $\mathrm{X}$ & $\mathrm{X}$ & $\mathrm{X}$ & $\mathrm{X}$ & $\mathrm{X}$ \\
\hline & $\begin{array}{l}\text { Performance } \\
\text { Evaluation }\end{array}$ & & & & & & $\mathrm{X}$ & & & $\mathrm{X}$ & & & & & \\
\hline & Application & & $\mathrm{X}$ & $\mathrm{X}$ & $\mathrm{X}$ & $X$ & $X$ & $X$ & $X$ & $X$ & $\mathrm{X}$ & $X$ & $X$ & $X$ & $X$ \\
\hline & $\begin{array}{l}\text { Intentional } \\
\text { Feedback }\end{array}$ & & & & & $\mathrm{X}$ & & & & & & & & & \\
\hline
\end{tabular}


The combined views of all study participants provided clear perspectives of what PBIS looks like (or does not look like) in each of their schools and at the district-level. Although the analysis and findings are limited to the individual schools that served as the context of my study, the ability to provide a glimpse into the inner workings of a specific group is beneficial to case study research (Compton-Lilly, 2013). Each school that participated offered a unique perspective of PBIS in action to inform district leadership on ways they can support the schools and the staff tasked with implementing PBIS with fidelity. The final chapter in this study will focus on the how this information can be used to improve policy and practice, not only throughout the FCSD, but also for any school district that plans to utilize PBIS as a district initiative. 


\section{CHAPTER V: DISCUSSION AND IMPLICATIONS}

The purpose of my multi-case embedded case study was to understand the perspectives of classified staff (and educational leaders) that work in a large urban school district that utilizes Positive Behavior Interventions and Supports (PBIS) as a districtwide initiative. My analysis revealed how this complex system normalizes within the district as part of their daily routines and work responsibilities using the Normalization Process Theory (NPT) as the guiding theoretical framework. The four components of NPT: coherence, cognitive participation, collective action, and reflexive monitoring were used to determine the effectiveness of PBIS within the district and provided an anchor on which to base the guiding questions and data analysis.

PBIS is a framework of evidence-based practices that provides "a three-tiered continuum of support to students, using systems to support staff in implementation, and using data for decision making” (McIntosh \& Goodman, 2016. p. 6). This school-wide systems approach teaches behavioral expectations as a way to prevent misbehaviors and

promote positive reinforcement (McIntosh \& Goodman, 2016). Additionally, the PBIS framework seeks to include all staff in the process to support students through common expectations both in the classroom and in non-classroom settings.

I focused on the perspectives of classified staff that are directly involved with students on a regular basis but are often not included in key areas of implementation within the school setting. I also looked at how district and school-level leaders include 
classified staff members in the overall initiative and support them as they implement PBIS at the school-level. The significance of gaining the perspective of classified staff is based on the following views:

- Many classified staff hold positions that place them in direct contact with students. Focus group participants in this study included office staff, cafeteria staff, instructional assistants, bilingual instructors, security monitors, mental health practitioners, home/school liaisons, and a former bus driver.

- School districts expect these staff members to intervene with students and follow school-wide expectations, but do not measure or evaluate their effectiveness consistently, nor are they involved in the same level of training.

- Classified staff interactions with students are different from their certified counterparts. They have opportunities for deeper relationships and mentoring while providing supports during interventions.

- Classified staff are important stakeholders in the school setting, and their inclusion in systemic initiatives is important in order for them to be actively engaged in programming that will allow for their professional growth.

Through this study, I provide a unique perspective from classified staff so often left out of research studies involving PBIS. In this chapter, I provide answers to my research question in summary form and discuss how these findings may improve the implementation of whole-district initiative through the inclusion of a key stakeholder 
group. The final section includes a discussion of implications for policy, practice, and future research.

\section{Discussion and Interpretation of Findings}

PBIS is a program used in over 20,000 schools nationwide and promotes changes in staff behavior that seek to improve student behaviors and student outcomes (McCurdy et al., 2016, Pas \& Bradshaw, 2012). Although literature calls for inclusion of all staff in the implementation of PBIS to ensure successful implementation over time (Mercer et al., 2017; McIntosh et al., 2016), the findings from this study did not align with this expectation. District and school leadership participants were able to provide specific information about the core components of PBIS; however, they did not have a fully developed strategy for ensuring that classified staff had the same consistent information that certified staff were privy to during their training.

Ensuring the successful implementation of PBIS is dependent on building capacity within and amongst school staff (Strickland-Cohen \& Horner, 2015), and this study supports this view. While participants held positive views of PBIS overall, the lack of in-depth understanding within the classified staff focus groups and the limited training opportunities provided by district and school leadership for classified staff does not prepare all staff for PBIS implementation. This discussion of findings focuses on the four components of NPT that guided this study as they relate to the everyday practices of the leaders and classified staff tasked with ensuring the districts mandate for PBIS.

According to May and colleagues (2015), understanding the differences between old and new practices, having a shared understanding of those practices, and finding 
value in those practices leads to organizational coherence, the first component of NPT. District and school leaders in the FCSD were clear about the variances in practices and understood the need to provide supports to staff to ensure the shared understanding for all staff. However, this did not translate to true understanding by classified staff members. Classified staff were implementing PBIS strategies based on second-hand accounts or overheard conversations outside of the initial beginning of year overview. This aligns with previous research studies by Bethune (2017) and Rodriguez, Loman, and Borgmeier (2016) that found that one time large group trainings and implementing PBIS before receiving adequate training was a barrier to PBIS success.

All school personnel need to be included in ongoing training over the course of implementation if fidelity of the initiative is expected (Lohrmann, Forman, Martin, \& Palmieri, 2008). This was not the case in the findings from this study. When asked about the inclusion of classified staff in specific PBIS training, both district and school leaders had minimal expectations for this process to occur regularly. While stating multiple reasons for this, the lack of funding, resources, and scheduling conflicts emerged as the most common issues for providing this support. This finding also aligned with previous research by Bubb and Earley (2013) who noted that the entirety of the staff is often neglected when funding training for personnel.

Large organizations, like FCSD, can ensure that all stakeholders have the opportunity to make sense of complex initiatives when the goal is to implement organizational change and weave it into normal staff practices. The classified staff that took part in this study made their own connections to PBIS as it related to their specific 
role within the school building. Increasing their overall comprehension of the purpose and intent of PBIS has to potential to improve outcomes for the district as a whole.

The second component of NPT is cognitive participation, which is the relational work that people do to build and sustain a community of practice (http/normalizationprocess.org/npt-toolkit). Prior studies indicated that classified staff assist in a variety of tasks that include clerical work, student monitoring in noninstructional areas, and behavior support (Barnes et al., 2018; Brown \& Staton-Chapman, 2017) and that all staff would be more productive when provided time to collaborate and attend professional development (Desimone \& Pak, 2017). The classified staff that participated in this study described their daily responsibilities and spoke clearly about the role they played in the day-to-day interventions with students, which included student interventions, de-escalation, monitoring common areas of the building, providing safe spaces for students who were in crisis, and supporting teachers when difficulties arose with students. Although they did not attend professional development with certified staff, they were willing to do whatever was necessary to assist with students even if they lacked the core strategic information about PBIS best practices.

This was corroborated by the district and school leaders who were in charge of professional development for schools throughout the district. Although these leaders expressed throughout the interview process that classified staff were integral to the overall school plan to support PBIS, there were no explicit plans to include them on a consistent basis. May and Finch (2009) emphasized the building relationships amongst the group to organize the belief in an intervention was needed to sustain the intervention. 
This is true for the FCSD as they build momentum for PBIS as a district mandate. The intentionality of leadership will be imperative as the district moves forward.

Collective Action is the third component of NPT and looks at the operational work that people do when engaging in a set of practices as a normal condition of their work. May and Finch (2009) recognized that the collectivity of participants allowed them to invest in the complexity of initiatives. As evidenced in this study, that collective actions of classified staff members at all three grade bands was a normal part of their daily job functions, even when they had minimal discussions or input from supervisors or other certified staff members.

The classified staff that participated in this study were committed to their support of the overall school culture and climate and developed their own systems and hierarchies as they worked collectively within their role group and team. All of the classified staff expressed a positive view of PBIS in general, which is key to the sustainability of PBIS within the district and aligns with the research that identifies an $80 \%$ commitment to a program in order to sustain the initiative within an organization (Vancel et al., 2016).

District and school leaders that participated in the study described their collective actions as developing overall plans for PBIS implementation, reviewing trend data to make informed decisions, and planning professional development for staff.

Unfortunately, this did not consistently include classified staff participation. To support a true collective effort for the FCSD, the district needs explicit plans to include all certified and classified staff in training, planning, and implementation of the PBIS initiative. 
The final component of NPT is reflexive monitoring, the ability to get feedback to assess and improve job performance for all staff. This area was the most lacking component in both the interviews and the focus group sessions. According to Fisher and Pleasant (2012), many classified staff members were often dissatisfied with their roles due to feeling marginalized and excluded from participation in planning. Although the classified staff that participated in this study did not indicate dissatisfaction with their jobs, they did indicate a lack of consistent feedback regarding their work from supervisors; they simply went about their day doing what they thought was best for students.

However, Stewart (2018) found that feedback on job performance for classified staff was essential to not only improving overall work quality but also that communicating clear role definition cleared up any ambiguity that classified staff felt regarding their job responsibilities. Both groups of participants, leadership and classified staff, shared a lack of clearly defined expectations for PBIS implementation when it came to classified staff job responsibilities. School leaders described informal feedback to classified staff as either situational or annual. Classified staff described feedback in reverse, with school administrators asking them for advice on how to work with certain students.

Consistent feedback loops were missing entirely from this study. Although professional development plans were in place for certified staff, classified staff were only included in the beginning of the year training. However, these trainings did not include feedback as a component for PBIS beyond reviewing trend data regarding student behavioral progress. The lack of reflexive monitoring as a whole does not align with the 
work of May and Finch (2009) who structured this component of NPT around effectiveness, worthiness, personal relationships within the intervention, and procedural improvements. District and school leaders acknowledged classified staff as integral parts of overall implementation but did not take time to provided adequate opportunities to help them improve their job performance when it came to PBIS implementation.

Overall, the FCSD mandated the PBIS initiative for all schools in the district and provided basic training opportunities for schools with recommendations for which staff members and stakeholders to include on individual PBIS committees at the school level. From there, schools determine team membership and plans to meet the needs of their particular schools and situations. The interviews and focus groups revealed a top-down approach to PBIS implementation within the school district but did not fully account for how classified staff should be included in the initiative. Although classified staff that participated in the study held an overall positive view of PBIS, it was evident that they gained their knowledge from 'overheard conversations' or their own natural ability to relate to students.

Much of the work that classified staff engaged in aligns with the core components of PBIS and with explicit training, inclusion in professional development, and consistent feedback opportunities, these particular staff members have the potential to positively impact school climate and culture. Overall, the classified staff that participated in the study were supportive of the PBIS initiative, even with their minimal inclusion in the process. Educational leaders should capitalize on eager and willing employees by providing classified staff a seat at the table. 


\section{Recommendations for Policy and Practice}

The findings from my study reveal important recommendations that may improve the implementation and impact of PBIS. PBIS research spanning the past 20 years calls for the inclusion of all stakeholders (Feuerborn \& Tyre, 2016; Filter et al, 2016;

Goodman-Scott et al., 2018; McIntosh et al, 2015). Additionally, research shows that classified staff play an important role in schools throughout the nation (Carter et al., 2009; Downing, Rydak, \& Clark, 2000; Fisher \& Pleasants, 2012; Giangreco, 2013). My analysis supports previous research and provides first-hand accounts of how classified staff work to support schools and students on a regular basis.

Using NPT as a conceptual map to evaluate PBIS within a district that oversees over 100,000 students and employs thousands of men and women may provide implications for policy and practices regarding the collective action of the people required to implement a complex intervention. First, classified staff are in positions that provide them opportunities to connect with students in ways that certified staff are not. Classified staff have a forward-facing nature within their roles in the office, cafeteria, classrooms, school halls, counseling office, home-school connections, and bus routes. Within these roles, classified staff are in positions that enable them to engage with students as soon as they begin their school day. This first interaction can either help or hinder a student's school day if a problem arises. Classified staff are also expected to intervene with students while following school-wide expectations for which they often have limited background knowledge. Both classified and certified staff acknowledged during the study that time and funding affected the ability to include classified staff in the training on a consistent basis. 
My analysis revealed that classified staff felt positive about the work that they do to support students, which was different from previous studies by Giangreco, Suter, and Doyle (2010), Fisher, and Pleasant (2012) that found more job dissatisfaction amongst classified staff based on a lack of clearly defined job descriptions and marginalization. They expressed that they feel respected, although their leadership do not hear their voices when it comes to specific strategies they use to work with students in need of support. Their unique interactions with students can be of benefit to certified staff when determining ways to deescalate intense situations when students misbehave. Both district and school-level leadership confirmed what classified staff expressed during focus groups that members of this particular role group (CS) have often developed deep relationships with students that positively impact student's decision making abilities.

In order to implement PBIS as a district initiative effectively, all staff members (certified and classified) should be included in all aspects of the initiative in order to see systemic success. Shared understanding and the ability to be actively engaged in programming, the ability to learn and grow based on specific feedback and monitoring, and full participation in complex initiatives are necessary to ensure successful implementation aligned with research by Strickland-Cohen and Horner (2015). The full inclusion of classified staff has the potential to increase the effectiveness of PBIS in FCSD and the students it serves and can be a model for other districts if consistent plans are developed.

This requires that districts ensure that funding is available to include classified staff members in PBIS training modules to build their background knowledge specific to the unique role that they occupy. FCSD leaves budgeting decisions regarding PBIS to 
the schools as an unfunded mandate. However, if schools truly are to be inclusive of all staff, decisions regarding training should ensure that time and funding are not a hindrance to programming. Classified staff in this study expressed that they wanted to be more involved in training because they have already "bought-in" to the program but lack of first-hand information and direct feedback from leadership that could help them to improve their job performance was limited.

District and school leaders will need to create a consistent plan that will allow all staff to be active participants in the implementation of PBIS in order to improve student outcomes. This plan must include an intentional focus on the inclusion of classified staff so that they have a full understanding of PBIS and their role in the daily implementation. PBIS can have a meaningful impact on improved student outcomes when implemented with consistency and clarity and when schools involve staff members in comprehensive training.

District-level resource teachers were available to schools and served as a direct link for feedback regarding survey results and school discipline data. However, neither school leaders nor classified staff mentioned the work of these resource teachers. None of the school-level staff seemed aware of the fact that the district has staff members whose specific role is to provide support to schools, suggesting a breakdown of practice. Schools must be knowledgeable of the resources that are available and utilize the expertise of these support people to reflect on and enhance their PBIS implementation.

Inclusion of classified staff on school-based PBIS teams should be required, not just recommended, to ensure that all perspectives are included during planning. Additionally, no one at the school-level mentioned PBIS action plans discussed during 
district leadership interviews. To have an effect, schools must be aware of and implement existing plans with consistent monitoring and updating. The district would be wise to collaborate with resource teachers, school leadership, and school-based PBIS teams in the provision of supports and data monitoring.

PBIS training for classified staff must be a priority for all school districts that utilize this initiative. While FCSD requires a training for PBIS at the beginning of the school year, the only participants who mentioned this during the study were school and district leadership. The fact that the classified participants did not recall such training or refer to anything they learned from the training shows that this one training requirement is not effective in making a long-term impact.

Finally, districts who wish to make PBIS a district-wide system should commit to common PBIS plans and practices that encompass multiple levels of training. This should include beginning-of-year trainings followed up with regular and consistent updates throughout the year. All staff, certified and classified, need to be included in trainings planned and scheduled to ensure the inclusion of all stakeholders. These trainings should take priority during full staff meetings and professional development days with specific time built in for classified staff to participate in planning to engage them in strategies that address their unique job roles.

\section{Implications for Future Research}

The findings in my study have implications for future research opportunities. The basis of this study was in response to the lack of PBIS research that included the views and perspectives of classified staff. It was evident throughout my study that the classified 
staff within FCSD were not a priority when planning PBIS training modules, even if it was not intentional. Future studies should focus on explicit plans that districts or schools develop to include classified staff members that will support them in PBIS implementation strategies. These plans should include nuances between certified and classified job responsibilities as it relates to the initiative. Through future research, classified staff could provide a voice that identifies methods that are effective within their daily interactions with students and bridge gaps between teachers and support staff.

Future research may want to explore the inclusion of classified staff in the budgeting process of district initiatives. Mandates to incorporate a program or initiative at full scale should be deliberate in the rollout and purposefully consider who should be involved at each layer of training. This requires looking at how districts design their budgets to include all stakeholders and is supported by prior research conducted by Bubb and Earley (2013) and Forman et al., (2009). Including classified staff and/or paraprofessionals is an essential part of changing an organizational system when the organization expects these staff members to implement a program along with the certified or professional staff. This should not be overlooked in the research on organizational change or programs like PBIS.

Finally, future studies may examine the specific roles that classified staff have in schools across the country. My study revealed evidence of relationships, mentorships, mental health supports, and safe spaces for students to deescalate and discuss problems. Classified staff members have perspectives about students and strategies that they use that can be beneficial to certified staff, especially as it relates to dealing with misbehavior. Although many classified staff do not hold licensures to teach, they often 
have other professional backgrounds that lend themselves to working with children in non-instructional roles. Learning from these classified staff members could add another layer of support for PBIS and other complex initiatives.

\section{Conclusion}

I sought to gain insight into how one school district includes their classified staff in the implementation process of PBIS as a district initiative. The interviews with district and school leadership revealed a top-down approach to implementation that did not fully account for how classified staff should be included in the initiative. Classified staff shared their perspectives of how they use PBIS strategies on a daily basis and were only able to articulate that the main purpose was to 'be positive' with students and keep students from being suspended from school.

What emerged from the data was the overall positive views of working with students who were experiencing behavioral issues in the school setting. All of the classified participants expressed their interests in supporting students and teachers and providing safe spaces for students to express themselves. This is a positive indicator for classified staff inclusion in PBIS training. Much of what they do aligns with the constructs of PBIS, and all that participated were willing to learn and improve their job performance as it relates to working with students. Because PBIS is about changing adult behavior prior to changing student behavior, classified staff are primed for learning. They value the work that they do, they value their schools, and they value their students.

It is important that district and school leaders include these key staff members in the overall PBIS plan as full participants and not just as cursory participants that build 
their knowledge base through hearsay. As members of the middle and high school focus groups expressed, they are bought-in, willing, and 'naturals' for PBIS implementation. It is what they do. If educational leadership wants to capitalize on eager and willing employees, then providing classified staff a seat at the table is critical and high priority. 


\section{REFERENCES}

Ajzen, I. (2002). Perceived behavioral control, self efficacy, locus of control, and the theory of planned behavior. Journal of Applied Social Psychology, 32(4), 665-683.

Andreou, T.E., McIntosh, K., Ross, S.W., \& Kahn, J. D. (2015). Critical incidents in sustaining school-wide positive behavioral intervention and supports. The Journal of Special Education, 49(3), 157-167.

https://doi.org/10.1177/0022466914554298

Bambara, L.M., Goh, A., Kern, L., \& Caskie, G. (2012). Perceived barriers and enablers to implementing individualized positive behavior interventions and supports in school settings. Journal of Positive Behavior Interventions, 14(4), 228-240. https://doi.org/10.1177/1098300712437219

Bandura, A. (2000). Exercise of human agency through collective efficacy. American Psychological Society, 9(3), 75-78.

Barnes, T.N., Cipriano, C., McCallops, K., Cuccuni-Harman, C., \& Rivers, S.E. (2018). Examining the relationship between perceptions of teacher self-efficacy, school support, and teacher and paraeducator burnout in a residential school setting. Emotional and Behavioral Difficulties, 23(3), 284-985.

https://doi.org/10.1080/1362752.2018.1461452 
Bethune, K.S. (2017). Effects of coaching teachers' implementation of tier 1 school-wide positive behavioral interventions and support strategies. Journal of Positive Behavior Interventions, 19(3), 131-142. https:/doi.org/10.1177/109830071668009

Bohanon, H., Fenning, P., Carney, K.L., Minnis-Kim, M.J., Anderson-Harris, S., Moroz, K.B., ..Pigott, T.D. (2006). Schoolwide application of positive behavior support in an urban high school: A case study. Journal of Positive Behavior Interventions, 8(3), 131145.

Brown, T.S. \& Stanton-Chapman, T.L. (2017). Experiences of paraprofessionals in US preschool special education and general education classrooms. Journal of Research in Special $\quad$ Educational Needs, 17(1), 18-30. https://doi.org/10.1111/14713802.12095

Bubb, S. \& Earley, P. (2013). The use of training days: finding time for teachers' professional development. Educational Research, 55(3), 236-248. https://doi.org/10.1080/00131881.2013.825161

Bunch-Crump, K.R. \& Lo, Y. (2017). An investigation of multitiered behavioral interventions on disruptive behavior and academic engagement of elementary students. Journal of Positive Behavior Interventions, 19(4), 216-227. https://doi.org/10.1177/109830071766939

Carter, E., O’Rourke, L., \& Sisco, L.G. (2009). Knowledge, responsibilities, and training needs of paraprofessionals in elementary and secondary schools. Remedial and Special Education, 30(6), 344-359. https://doi.org/10.1177/0741932508324399 
Clara, M. (2016). Teacher resilience and meaning transformation: How teachers reappraise situations of adversity. Teacher and Teacher Education, 63, 82-91. https://doi.org/10.1016/j.tate.2016.12.010

Compton-Lily, C. (2013). Case Studies. In Trainor, A.A \& Graue, E. (Eds.) Reviewing Qualitative Research in the Social Sciences (pp. 54-65). New York, NY:

Routledge.

Creswell, J. W. (2014). Research Design; Qualitative, Quantitative, and Mixed Methods Approaches. Thousand Oaks, CA: Sage.

DePrince, A.P., Weinzierl, K.M., Combs, M.D. (2009). Executive function performance and trauma exposure in a community sample of children. Child Abuse \& Neglect, 33, 353- 361. https://doi.org/10.1016/j.chiabu.2008.08.002

Desimone, L.M. \& Pak, K. Instructional coaching as high-quality professional development. Theory into Practice, 56, 3-12.

https://doi.org/10.1080/00405841.2016.1241947

Downing, J.E., Ryndak, D.L., \& Clark, D. (200). Paraeducators in inclusive classrooms: Their own perceptions. Remedial and Special Education, 21(3), 171-181.

Duncan, A. (2009). Letter to Chief State School Officers regarding the use of seclusion and restraint techniques in school. Retrieved from U.S. Department of Education website: $\quad$ http://www2.ed/print/policy/elsec/guid/secletter/9-731.html Durlak, J.A., Weissberg, R.P., Dymnicki, A.B., Taylor, R. D., \& Schellinger, K.B. (2011). The impact of enhancing students' social and emotional learning: A meta- 
analysis of school- $\quad$ based universal interventions. Child Development, 82(1), 405-432. https://doi.org/10.1111/j.1467/8624.2010.01564x

Dyson A.H. \& Genishi, G. (2005). The Case: Approaches to Language and Literacy Research. New York, NY. Teachers College Press.

Fay, J. \& Funk, D. (1995). Teaching with Love \& Logic. Golden, CO: Love and Logic Press

Feuerborn, L.L. \& Tyre, A.D. (2016). How do staff perceive schoolwide positive behavior supports? Implications for teams in planning and implementing schools.

Preventing School Failure, 60(1), 53-59.

https://doi.org/10.1080/1045988X.2014.974489

Feuerborn, L.L., Tyre, A.D., \& Beaudoin, K. (2018). Classified staff perceptions of behavior and discipline: Implications for schoolwide positive behavior supports. Journal of Positive Behavior Interventions, 20(2), 101-112. https://doi.org/0.1177/1098300717733975

Filter, J.K., Sytsma, M.R., \& McIntosh, K. (2016). A brief measure of staff commitment to implement school-wide positive behavioral interventions and supports.

Assessment for $\quad$ Effective Intervention, 42(1), 18-31.

https:/doi.org/10.1177/1534508416642212

Fisher, M. \& Pleasants, S.L. (2012). Roles, responsibilities, and concerns of paraeducators: $\quad$ Findings from a statewide survey. Remedial and Special Education, 33(5), 287-297. https://doi.org/10.1177/0741932510397762 
Freeman, J.\& Sugai, G. (2013). Recent changes in state policies and legislation regarding restraint and seclusion. Exceptional Children, 79(4), 427-438.

French, N.K. (1998). Working together: Resource teachers and paraeducators. Remedial and Special Education, 19(6)357-368.

Fullen, M. \& Quinn, J. (2016). Coherence: The Right Drivers in Action for Schools, Districts, $\quad$ and Systems. Thousand Oaks, CA. Sage.

Hydén, L.-C. \& Bülow, P.H. (2003). Who’s Talking: drawing conclusions from focus groups- $\quad$ some methodological considerations. International Journal of Social Research Methodology, ISSN 1364-5579. https://doi.org/10.1080/13645570210124865.

Hoffman, L. (2011). A federal solution that falls short: Why the Keeping All Students Safe Act fails children with disabilities. Journal of Legislation 37(1).

Horner, R.H., Dunlap, G., Koegel, R.L., Carr, E.G., Sailor, W., et al. (1990). Toward a technology of “nonaversive” behavioral support. Journal of the Association for Persons $\quad$ with Severe Handicaps, 15, 125-132.

Gage, N.A., Whitford, D.K., \& Katsiyannis, A. (2018). A review of schoolwide positive behavior interventions and supports as a framework for reducing disciplinary exclusions. The Journal of Special Education, 1-10. https://doi.org/10.1177/0022466918767847 
Garwood, J.D., Van Loan, C.L., Werts, M. (2018). Mindset of paraprofessionals serving students with emotional and behavioral disorders. Intervention in School and Clinic, 53(4), 206- 211. https://doi.org/10.1177/1053451217712958

Ghere, G. \& York-Barr, J. (2007). Paraprofessional turnover and retention in inclusive programs: Hidden costs and promising practices. Remedial and Special Education, 28(1), 21-32.

Giangreco, M. F., Sutter, J.C., \& Doyle, M.B. (2010). Paraprofessionals in inclusive schools: A review of recent research. Journal of Educational and Psychological Consultation, 20, 41-57. https://doi.org/10.1080/10474410903535356

Giangreco, M.F., Doyle, M.B., \& Sutter, J.C. (2012). Constructively responding to requests for paraprofessionals: We keep asking the wrong questions. Remedial and Special Education, 33(6), 362-373. https://doi.org/10.1177/0741932511413472

Giangreco, M.F. (2013). Teacher assistant supports in inclusive schools: Research, practices and alternatives. Australian Journal of Special Education, 37(2), 93-106. https://doi.org/10.1017/jse.2013.1

Goodman-Scott, E., Hays, D.G., \& Cholewa, B. E. (2018). It takes a village: A case study of positive behavioral interventions and supports implementation in an exemplary urban middle school. Urban Review, 50, 97-122. https://doi.org/10.1007/s11256-0170431-z

Hill, D.A. \& Flores, M. M. (2014). Modeling positive behavior interventions and supports for preservice teachers. Journal of Positive Behavior Interventions, 16(2), 93101. https://doi.org/10.1177/1098300713478665 
Howard, G. R. (2006). We Can't Teach What We Don't Know: White Teachers, Multiracial Schools. New York, NY: Teachers College Press

H.R. $4365-106^{\text {th }}$ Congress: Children's Health Act of 2000. (enacted)

Jolstead, K.A., Caldarella, P., Hansen, B., Korth, B.B., Williams, L., \& Kamps, D. (2017). Implementing positive behavior support in preschools: An exploratory study of CW-FIT T tier I. Journal of Positive Behavior Interventions, 19(1), 48-60. https://doi.org/ 10.1177/1098300716653226

Jones, C.R., Ratcliff, N.J., Sheehan, H., \& Hunt, G.H. (2012). An analysis of teachers’ and paraeducators’ roles and responsibilities with implications for professional development. Early Childhood Education Journal, 40 (9-24). https://doi.org/10.1007/s10643-011-0487- 4

Kitzinger, J. and Barbour, R.S. (1999). Introduction: The challenge and promise of focus groups. $\quad$ In R.S. Barbour and J. Kitzinger (eds) Developing Focus Group Research: Politics, $\quad$ Theory, and Practice. London: Sage

Lambert, S.D. and Liselle, C.G. (2008). Combining individual interviews and focus groups to enhance data richness. The Authors. Journal compilation 11(9).

Lohrmann, S., Forman, S., Martin, S., \& Palmieri, M. (2008). Understanding school personnel's resistance to adopting schoolwide positive behavior support at a universal level of intervention. Journal of Positive Behavior Interventions, 10(4), 256-269. https://doi.org/10.1177/1098300708318963 
Llyod, A., Joseph-Williams, N., Edwards, A., Rix, A., \& Elwyn, G. (2013). Patchy coherence: Using normalization process theory to evaluate a multi-faceted shared decision making implementation program (MAGIC). Implementation Science 8(102). https://doi.org/10.1186/1748- 5909-8-102

Madigan, K., Cross, R.W., Smolkowski, K., \& Stryker, L.A. (2016). Association between schoolwide positive behavioral interventions and supports and academic achievement: a $\quad$ 9-year evaluation. Educational Research and Evaluation, 22(7-8), 402-421. $\quad$ https://doi.org/10.1080/13803611.2016.1256783

Malinen, O. \& Savolainen, H. (2016). The effect of perceived school climate and teacher efficacy in behavior management on job satisfaction and burnout: A longitudinal study. Teacher and Teacher Education, 60, 144-152.

Martin, T. \& Alborz, A. (2014). Supporting the education of pupils with profound intellectual and multiple disabilities: the views of teaching assistants regarding their own learning and developmental needs. British Journal of Education, 41(3), 310-326. https://doi.org/ 10.1111/1467-8578.12070

May, C. (2013). Towards a general theory of implementation. Implementation Science, 8(18), 1- 14. Retrieved from http://www.implemenationscience.com/content/8/1/18. May, C. \& Finch, T. (2009). Implementing, embedding, and integrating practices: An outline of normalization process theory. Sociology, 43(3), 535-554. https://doi.org/10.1177/0038038509103208 May, C., Rapley, T., Mair, F.S., Treweek, S., Murray, E., Ballini, L., Macfarlane, A. Girling, M. \& Finch, T.L. (2015) Normalization Process Theory On-line Users' 
Manual, Toolkit and NoMAD instrument. Available from http://www.normalizationprocess.org

May, C., Cummings, A., Girling, M., Bracher, M., Mair, F.S., May, C.M., Murry, E. ... \& Finch, T. (2018). Using Normalization process theory in feasibility studies and process evaluations of complex healthcare interventions: a systemic review. Implementation Science 13(80). https://doi.org/10.1186/s13012-018-0758-1 McCurdy, B.L., Thomas, L., Truckenmiller, A., House-Rich, S., Hillis-Clark, P., \& Lopez, J.C. (2016). School-wide positive behavior interventions and supports for students with emotional and behavioral disorders. Psychology in the Schools, 53(4), 375-389. https://doi.org/10.1002/pits.21913

McKenzie, B. (2011). Empowering paraprofessionals through professional development. The Delta Kappa Gamma Bulletin, 38-41.

McIntosh, K., Chard, D.J., Boland, J.B., \& Horner, R.H. (2006). Demonstration of combined efforts in school-wide academic and behavioral systems and incidents of reading and behavior challenges in early elementary grades. Journal of Positive Behavior Interventions, 8(1), 146-154.

McIntosh, K., Mercer, S.H., Nese, R.N.T., Stickland-Cohen, K., \& Hoselton, R. (2016). Predictors of sustained implementation of school-wide positive behavioral interventions and supports. Journal of Positive Behavior Interventions, 18(4), 209-218. https://doi.org/10.1177/1098300715599737 
Merida-Lopez, S. \& Extremera, N. (2017). Emotional intelligence and teacher burnout: A systemic review. International Journal of Educational Research, 85, 121-130. https://doi.org/10.1016/j.oker.2017.07.006

Mercer, S.H., McIntosh, K, \& Hoselton, R. (2017). Comparability of fidelity measures for assessing tier 1 school-wide positive behavioral interventions and supports. Journal of Positive Behavior Interventions, 19(4), 195-204. https://doi.org/10.1177/1098300717693384

Miles, M. B., Huberman, A.M., \& Saldaña, J. (2014). Qualitative Data Analysis, A Methods Sourcebook. Thousand Oaks, CA: Sage.

Milner, H. R. (2007). Race, culture, and researcher positionality: Working through dangers seen, unseen, and unforeseen. Educational Researcher, 36(7), 388-400. https://doi.org/10.3102/001389X07309471

Moore, S. \& Kochan, F. (2013). Principals’ perceptions of professional development in high- and low-performing high-poverty schools. International Journal of Educational Reform, 22(2), 167-181.

Neese, R., McIntosh, K., Nese, J., Hoselton, R., Bloom, J., Johnson, N., ...Ghemraoui, A. (2016). Predicting abandonment of school-wide positive behavioral interventions and $\quad$ supports. Behavioral Disorders, 42(1), 261-270.

Normalization Process Theory, (2018). NPT Core Component. Retrieved from http:/www.normalizationprocess.org/npt-toolkit. 
Pas, E.T., Johnson, S.R., Debnam, K.J., Hullerman, C.S., \& Bradshaw, C. P. (2019). Examining the relative utility of PBIS implementation fidelity scores in relation to student outcomes. Remedial and Special Education, 40(1), 6-15. https://doi.org/0.117/0741932518805192

Pinkleman, S.E., McIntosh, K., Rasplica, C.K., Bert, T., \& Strickland-Cohen, M.K. (2015). Perceived enablers and barriers to sustainability of school-wide positive behavioral interventions and supports. Behavioral Disorders, 40(3), 171-183.

Raimundo, R., Marques-Pinto, A., \& Lima, M.L. (2012). The effects of a socialemotional learning program on elementary school children: The role of pupil’s characteristics. Psychology in the Schools, 50(2), 165-180. https://doi.org/10.1002/pits.21667

Reno, G.D., Friend, J., Caruthers, L., \& Smith, D. (2017). Who’s getting targeted for behavioral interventions? Exploring the connection between school culture, positive behavior support, and elementary student achievement. The Journal of Negro Education, 86(4), $\quad$ 423-438.

Riggs, C.G. \& Mueller, P.H. (2001). Employment and utilization of paraeducators in inclusive $\quad$ settings. The Journal of Special Education, 35(1), 54-62.

Rodriguez, B. Loman, S.L., \& Borgmeier, C. (2016). Tier 2 interventions in positive behavior support: A survey of school implementation. Preventing School Failure: Alternative Education for Children and Youth, 60(2), 94-105. https://doi.org/ 10.1080/1045988X.2015.10525354

S. 2020- $112^{\text {th }}$ Congress: Keeping All Students Safe Act. (2011) 
Scott, T. M. (2012). Universal systems for preventing behavior problems (pp. 191-216).

In B. G.

Cook, M. Tankersley, \& T. J. Landrum (Eds.), Classroom behavior, contexts, and interventions (Advances in learning and behavioral disabilities, Volume 25).

Bingley,

UK: Emerald Publishing Group

Scott, T.M. \& Hirn, G.H. (2013). Addressing challenging behavior: Considering logic of probability. Intervention in School and Clinic, 27, 1-5.

https://doi.org/10. 1177/1053451213493169

Shyman, E. (2010). Identifying predictors of emotional exhaustion among special education paraeducators: A preliminary investigation. Psychology in the Schools, 47(8), 828-841. https://doi.org/10.1002/pits.20507

Simonsen, B. \& Myers, D. (2015), Classwide Positive Behavior Interventions and Supports: A Guide to Proactive Classroom Management. New York, NY. Gilford Press.

Singer, G. H., \& Wang, M. (2009). The intellectual roots of positive behavior support and their implications for its development. In W. Sailor, G. Dunlap, G. Sugai, \& R. Horner (Eds.), Handbook of positive behavior support (pp. 17-48). New York: Springer

Song, H., Hur, E., \& Kwon, B. (2017). Does high-quality professional development make a difference? Evidence from TIMSS. Compare: A Journal of Comparative and 
International Education, 48(6), 954-972.

https://doi.org/10.1080/03057925.2017.1373330

Stewart, E.M. (2018). Reducing ambiguity: Tools to define and communicate paraprofessional roles and responsibilities. Intervention in School and Clinic, 1-7. https://doi.org/10.1177/1053451218782431

Stockwell, N.S. (2014). When an aide really becomes and aide: Providing professional development for special education paraprofessionals. Teaching Exceptional Children, ～46(6), 197-205. https://doi.org/10.1177/0040059914537202

Strickland-Cohen, M.K. \& Horner, R.H. (2015). Typical school personnel developing and implementing basic behavior support plans. Journal of Positive Behavior Interventions, 17(2), 83-94. https://doi.org/10.1177/1098300714554714

Sprick, R.S., Sprick, M., \& Garrison, M. (1992). Foundations: Establishing positive discipline policies. Longmont, CO: Sopris West.

Taylor, R. D., Oberle, E., Durlak, J.A., \& Weissberg, R.P. (2017). Promoting positive youth development through school-based social and emotional interventions: A metaanalysis $\quad$ of follow-up effects. Child Development, 88(4), 1156-1171. https://doi.org/10.1111/cdev.12864

Torrente, C.E., Cappella, E., \& Watling-Neal, J. (2014). Children’s positive school behaviors and social preference in urban elementary classrooms. Journal of Community Psychology, 42(2), 143-161. https://doi.org/10.1002/jcop.21599 
Vancel, S.M., Missall, K.N., \& Bruhn, A.L. (2016). Teacher ratings of the social validity of schoolwide positive behavior interventions and supports: A comparison of school groups. $\quad$ https://doi.org/10.1080/1045988X.1157784

U.S. Department of Education, Guiding Principles: A Resource Guide for Improving School Climate and Discipline, Washington, D.C., 2014

Ward, B. \& Gersten, R. (2013). A randomized evaluation of the safe and civil schools model for positive behavioral interventions and supports at elementary schools in a large urban school district. School Psychology Review, 42(3), 317-333.

Weiner, B.J. (2009). A theory of organizational readiness for change. Implementation Science, $\quad 4(67)$. Retrieved from http:/www.implementationscience.com/content/4/1/67.

Whitaker, T. (2013). What Great Teachers Do Differently: 17 Things That Matter Most. New York, NY: Rutledge

Yin, R. K. (2018). Case Study Research and Applications. Design and Methods. Los Angeles, CA: Sage. 


\section{Project Title: INCLUSION OF CLASSIFIED STAFF IN THE IMPLEMENTATION OF POSITIVE BEHAVIOR INTERVENTIONS AND SUPPORTS: A DISTRICT CASE STUDY}

\section{Investigator(s) name \& address:}

Co-Advisor and Principal Investigator:

W. Kyle Ingle, Ph.D.

College of Education and Human Development

University of Louisville

1905 South 1st Street

Louisville, KY 40292

william.ingle@louisville.edu

Devon M. Roberts M. Ed.

University of Louisville

4832 South $5^{\text {th }}$ St.

Louisville, KY 40214

devon.roberts@louisville.edu

Site(s) where study is to be conducted: University of Louisville, Jefferson County Public Schools.

Phone number for subjects to call for questions: W. Kyle Ingle (502) 852-6097

\section{Introduction and Background Information}

You are invited to participate in a research study. The study is being conducted by Devon Roberts under the supervision of Kyle Ingle, Ph.D, in Educational Leadership from Florida State University . This study is sponsored by the University of Louisville, Department of Education Leadership, Evaluation, and Organizational Development. The study will take place at the University of Louisville and Jefferson County Public Schools. Approximately 20 subjects will be invited to participate. 


\section{Purpose}

The purpose of this study is to study will examine the ways in which a large urban school district includes classified support staff in the training and implementation of Positive Behaviors Supports and Interventions.

\section{$\underline{\text { Procedures }}$}

In this study, you will be asked to provide demographic information and information about your experiences and involvement in PBIS training and initiatives. You will also be asked to provide responses to several questions about your experiences working with PBIS interventions as part of your daily responsibilities. Your participation will include a 60-minute individual interview or focus group to collect demographic and some contextual information. The interview will be audio recorded. I am highly flexible and am willing to meet with at your convenience. You may decline to answer any questions that make you feel uncomfortable.

\section{$\underline{\text { Potential Risks }}$}

There are no foreseeable risks other than possible discomfort in answering personal questions.

\section{$\underline{\text { Benefits }}$}

The possible benefits of this study to the participants include the opportunity for them to voice their perceptions and understanding of the collaborative efforts between districtlevel and school-level leadership that directly affects their roles and responsibilities when implementing PBIS. Additionally, this opportunity to participate may ensure that classified staff are able to express their opinions regarding their abilities to serve as drivers of student achievement through the use of PBIS as an intervention.

\section{Compensation}

You will not be paid for your time, inconvenience, or expenses while you are in this study.

\section{Confidentiality}


Total privacy cannot be guaranteed. We will protect your privacy to the extent permitted by law. If the results from this study are published, your name will not be made public. Once your information leaves our institution, we cannot promise that others will keep it private.

Your information may be shared with the following:

- The sponsor (Dr. W. Kyle Ingle) and others hired by the sponsor to oversee the research

- Organizations that provide funding at any time for the conduct of the research.

- The University of Louisville Institutional Review Board, Human Subjects Protection Program Office, Privacy Office, others involved in research administration and research and legal compliance at the University, and others contracted by the University for ensuring human subjects safety or research and legal compliance

- The local research team

- People who are responsible for research, compliance and HIPAA/privacy oversight at the institutions where the research is conducted

- Applicable government agencies, such as:

o Office for Human Research Protections

o Office of Civil Rights

\section{$\underline{\text { Conflict of Interest }}$}

This study involves no foreseeable conflict of interest.

\section{$\underline{\text { Security }}$}

The data collected about you will be kept private and secure by a password- protected computer and will be destroyed after the study is complete.

\section{Voluntary Participation}

Taking part in this study is completely voluntary. You may choose not to take part at all. If you decide not to be in this study, you won't be penalized or lose any benefits for which you qualify. If you decide to be in this study, you may change your mind and stop taking part at any time. If you decide to stop taking part, you won't be penalized or lose any benefits for which you qualify. You will be told about any new information learned during the study that could affect your decision to continue in the study. 


\section{U.S. Department of Education (DOE) Funded Studies}

Because school system receives funding from the DOE, we are required to tell you the following information.

The information we collect from the study may only be used to meet the purposes of the study as stated in this consent. We will conduct this study in a manner that does not allow identification of you by anyone other than study team members or others who may have a legitimate reason to know. All instructional materials or survey instruments used for the research are available for you to see before the study begins if you ask to see it. If you want to see any of this information, please contact Devon Roberts, (502) 819-1083 and she will give you a date and time where it will be available for you to review. Once the study is completed, we are required by the U.S. Department of Education to destroy or return to the school system all personally identifiable information when no longer needed for the purposes of the study. We expect this study to last for seven months and when the study is finished, we will delete any identifying information. All digital recordings will be destroyed by 2022 and all digital transcriptions will destroyed by 2026 .

\section{Contact Persons, Research Subject’s Rights, Questions, Concerns, and Complaints}

If you have any concerns or complaints about the study or the study staff, you have three options.

You may contact the principal investigator at (502) 852-6097 or william.ingle@louisville.edu

If you have any questions about your rights as a study subject, questions, concerns or complaints, you may call the Human Subjects Protection Program Office (HSPPO) (502) 852-5188. You may discuss any questions about your rights as a subject, in secret, with a member of the Institutional Review Board (IRB) or the HSPPO staff. The IRB is an independent committee composed of members of the University community, staff of the institutions, as well as lay members of the community not connected with these institutions. The IRB has reviewed this study.

If you want to speak to a person outside the University, you may call 1-877-852-1167. You will be given the chance to talk about any questions, concerns or complaints in 
secret. This is a 24-hour hot line answered by people who do not work at the University of Louisville.

\section{Acknowledgment and Signatures}

This document tells you what will happen during the study if you choose to take part. Your signature and date indicates that this study has been explained to you, that your questions have been answered, and that you agree to take part in the study. You are not giving up any legal rights to which you are entitled by signing this informed consent document though you are providing your authorization as outlined in this informed consent document. You will be given a copy of this consent form to keep for your records.

Relationship of Legal Representative to Subject

Printed Name of Person

Explaining Consent Form
Signature of Person Explaining Consent Form (if other than the Investigator) 


\section{APPENDIX B: RECRUITMENT CORRESPONDENCE}

Hello

I hope this e-mail finds you well. My name is Devon Roberts, and I am a Doctoral Candidate at the University of Louisville. I am writing as I am conducting a case study on PBIS as a district wide intervention and believe you would be able to provide critical insight.

The primary goal of my study is to investigate the inclusion of classified staff in the inclusion and implementation of PBIS through the lens of the normalization process theoretical framework. Thus, I am seeking to interview district and school-level leadership and classified staff who are involved in the implementation of PBIS. Your voice is of considerable importance to the study. My hope is that I might speak with you in person to gain a better understanding about your own personal experiences with PBIS.

I am asking that you participate in a 60-minute interview. My schedule to conduct an interview with you is flexible and can be scheduled at a time, date, and location of your convenience. If you have additional questions, please contact me via e-mail at devon.roberts@louisville.edu or call (502)819-1083.

Thank you in advance and I look forward to your response.

Sincerely,

Devon M. Roberts

Doctoral Candidate, Educational Leadership \& Organizational Development University of Louisville 
APPENDIX C: INTERVIEW PROTOCOL

\section{INTERVIEW PROTOCOL - DISTRICT LEADERSHIP \#1 \\ PBIS Implementation with Classified Staff}

Stage I: Introductions \& Statement of Purpose. (10 minutes)

Time Started:

Thank you for taking part in our focus group today. I am . I am conducting research on the implementation of PBIS and the inclusion of classified staff within this intervention. One of the ways I am doing this is by conducting interviews with district leadership involved in PBIS implementation. The purpose of this interview is to understand your perceptions and experiences with PBIS as part of your daily work to ensure district wide implementation. The feedback that you provide me today will help me to evaluate the quality of the PBIS training and supports to improve the delivery professional development for this project and future efforts. This interview offers me an opportunity to impact the future of the profession.

I also want to let you know that I will be recording our conversations. The recording will be transcribed by the transcription service, Rev.com. I will be analyzing the transcripts as part of my data analysis. I have informed consent forms here for your review and signature. But to sum it up, your identity will not be revealed in any reports, conference presentations or publications that might result from this study. A pseudonym will be used to identify you, your school, and district in any conference presentations of this research project or any manuscripts submitted for publication. The principal investigator and I am the only one who will have access to the file linking the participants with the pseudonyms. All documents and data collected will be kept in my locked office and password protected district issued computers. Files will be destroyed no later than January 1, 2022.

\section{[ALLOW TIME FOR REVIEW OF INFORMED CONSENT, QUESTIONS, AND SIGNATURES]}

Before we begin, please share your name and your role within the district. 


\section{Stage II: Ground Rules (10 minutes)}

I am certainly glad that you are able to participate and I and value the work that you do for schools. Before we begin, I want to lay down some ground rules for our discussion.

- I want you to know that I am mindful of your time and plan on keeping us for 60 minutes. I have purposely kept the number of questions short for this purpose.

- There are no right or wrong answers to the questions I will ask.

- As facilitator, I might move our conversations along to ensure that we cover all of the questions that we have in the interest of time.

- It is safe for you to freely express your opinions. Your experiences and opinions are important.

- Please feel free to ask questions for clarity if you don’t understand something.

Are there any questions so far?

\section{Stage III: Interview Questions (40-50 minutes)}

If there are no further questions or concerns, we will start with the questions.

1. Please tell me about your background as it relates to your position and role within the district.

2. What are your understandings of the goals and expectations of Positive Behavior Interventions and Supports (PBIS) and how is this shared throughout the district?

a. What are your roles and responsibilities of PBIS on a daily basis?

b. What are your shared values and beliefs of PBIS within the district?

3. How do you determine the professional development needs that will ensure that PBIS is implemented consistently throughout the district?

4. Are classified staff considered when developing training and support for PBIS implementation?

a. What does this look like?

5. How do you adjust PBIS training for classified staff and their various role groups?

6. How are budgetary decision made in regard to PBIS training for classified staff?

7. How do you define the involvement of classified staff in PBIS implementation throughout the district?

8. How do classified staff build their knowledge base of PBIS components?

9. How do classified staff receive feedback on their implementation of PBIS strategies? 


\section{Stage IV: Closure (5-10 minutes)}

Well that is all I have for you today. Before we conclude, are there any questions whatsoever? Do you have recommendations for other people I can speak to regarding this topic? [PROVIDE TIME FOR ANY QUESTIONS]

Again, we thank you for your willingness to participate in this interview and let your voice be heard. 


\section{INTERVIEW PROTOCOL - SCHOOL LEADERSHIP \#1}

PBIS Implementation with Classified Staff

Thank you for taking part in our focus group today. I am I am conducting research on the implementation of PBIS and the inclusion of classified staff within this intervention. One of the ways I am doing this is by conducting interviews with school leadership involved in PBIS implementation. The purpose of this interview is to understand your perceptions and experiences with PBIS as part of your daily work to ensure school wide implementation. The feedback that you provide me today will help me to evaluate the quality of the PBIS training and supports to improve the delivery professional development for this project and future efforts. This interview offers me an opportunity to impact the future of the profession.

I also want to let you know that I will be recording our conversations. The recording will be transcribed by a professional transcriptionist. I will be analyzing the transcripts as part of my data analysis. I have informed consent forms here for your review and signature. But to sum it up, your identity will not be revealed in any reports, conference presentations or publications that might result from this study. A pseudonym will be used to identify you, your school, and district in any conference presentations of this research project or any manuscripts submitted for publication. The principal investigator and I am the only one who will have access to the file linking the participants with the pseudonyms. All documents and data submitted to the evaluators will be kept in my locked office and password protected district issued computers. Files will be destroyed no later than January 1, 2022.

\section{[ALLOW TIME FOR REVIEW OF INFORMED CONSENT, QUESTIONS, AND SIGNATURES]}

Before we begin, please share your name, your school, and your role within the school. 


\section{Stage II: Ground Rules (10 minutes)}

I am certainly glad that you are able to participate and I value the work that you do for schools. Before we begin, I want to lay down some ground rules for our discussion.

- I want you to know that I am mindful of your time and plan on keeping us for 60 to 90 minutes. I have purposely kept the number of questions short for this purpose.

- There are no right or wrong answers to the questions I will ask.

- Since we have limited time, I'll ask that questions or comments off the topic be discussed after the interview.

- As facilitator, I might move our conversations along to ensure that we cover all of the questions that we have in the interest of time.

- It is safe for you to freely express your opinions. Your experiences and opinions are important.

- Please feel free to ask questions for clarity if you don't understand something.

Are there any questions so far? 


\section{Stage III: Interview Questions (40-50 minutes)}

If there are no further questions or concerns, we will start with the questions.

1. Please tell me about your background as it relates to your position as a school leader.

2. What are your understandings of the goals and expectations of Positive Behavior Interventions and Supports (PBIS) and how is this shared with the staff?

a. What are your roles and responsibilities of PBIS on a daily basis?

b. What are your shared values and beliefs of PBIS within the district?

3. How do classified staff interact with colleagues and supervisors to implement PBIS?

4. How are classified staff considered when developing training and support for PBIS implementation?

5. How is PBIS integrated into the daily work of classified staff within the school?

6. How do adjust PBIS training for classified staff and their various role groups?

7. How are budgetary decision made in regard to PBIS training for classified staff?

8. How do you define the involvement of classified staff in PBIS implementation on a daily basis?

9. How do classified staff build their knowledge base of PBIS components?

10. How do classified staff receive feedback on their implementation of PBIS strategies?

\section{Stage IV: Closure (5-10 minutes)}

Well that is all I have for you today. Before we conclude, are there any questions whatsoever? [PROVIDE TIME FOR ANY QUESTIONS]

Again, we thank you for your willingness to participate in this interview and let your voice be heard. 
APPENDIX D: FOCUS GROUP PROTOCOL

FOCUS GROUP PROTOCOL

PBIS Implementation with Classified Staff

Stage I: Introductions \& Statement of Purpose. (10 minutes)

Time Started:

Thank you for taking part in our focus group today. I am . I am conducting research on the implementation of PBIS and the inclusion of classified staff within this intervention. One of the ways I am doing this is by convening focus groups of classified staff like yourselves. The purpose of this focus group is to understand your perceptions and experiences with PBIS as part of your daily work within schools. The feedback that you provide me today will help me to evaluate the quality of the PBIS training and supports to improve the delivery professional development for this project and future efforts. This focus group offers me an opportunity to impact the future of the profession.

I also want to let you know that I will be recording our conversations. The recording will be transcribed by a professional transcriptionist. I will be analyzing the transcripts as part of my data analysis. I have informed consent forms here for your review and signature. But to sum it up, your identity will not be revealed in any reports, conference presentations or publications that might result from this study. A pseudonym will be used to identify you, your school, and district in any conference presentations of this research project or any manuscripts submitted for publication. The principal investigator and I am the only one who will have access to the file linking the participants with the pseudonyms. All documents and data submitted to the evaluators will be kept in my locked office and password protected district issued computers. Files will be destroyed no later than January 1, 2022.

\section{[ALLOW TIME FOR REVIEW OF INFORMED CONSENT, QUESTIONS, AND SIGNATURES]}

Before we begin, let us go around the room for some brief introductions. Please share your name, your school, and grade level that you serve. 


\section{Stage II: Ground Rules (10 minutes)}

Thanks for introducing yourselves. I am certainly glad that you are here and value the work that you do in schools. Before we begin, I want to lay down some ground rules for our discussion.

- I want you to know that I am mindful of your time and plan on keeping us for 60 minutes. I have purposely kept the number of questions short for this purpose.

- I want you to do most of the talking. I'd like to hear everyone speak so I might ask people who have not spoken up to comment.

- There are no right or wrong answers to the questions I will ask.

- As facilitator, I might move our conversations along to ensure that we cover all of the questions that we have in the interest of time.

- It is safe for you to freely express your opinions. Every person's experiences and opinions are important.

- All information shared during this focus group should remain confidential.

- Feel free to speak up whether you agree or disagree. Indeed, we want to hear a wide range of responses and opinions.

- However, please respect each other's opinions.

- Please speak one at a time and don't try and talk at one time or over one another. Please avoid side conversations too. These can have an impact on the quality of the recording and transcriptions that will result from our conversations.

- Please feel free to ask questions for clarity if you don’t understand something.

Are there any questions so far? 


\section{Stage III: Focus Group Questions (40-50 minutes)}

If there are no further questions or concerns, we will start with the questions.

1. What are your understandings of the goals and expectations of Positive Behavior Interventions and Supports (PBIS) and how is this shared with the staff?

a. What are your roles and responsibilities of PBIS on a daily basis?

b. What are your shared values and beliefs of PBIS within the school?

c. Who are the key leaders who drive the work of PBIS at your school?

2. How do you interact with colleagues and supervisors to ensure PBIS is implemented consistently?

3. How is PBIS integrated into your daily work?

4. How do you feel about your involvement in PBIS implementation?

5. How do you view your effectiveness with PBIS implementation?

a. How to you evaluate your PBIS practice?

b. How do you view your capacity to work with children using PBIS strategies?

c. How do you receive feedback on your implementation of PBIS strategies?

6. How do you define your involvement in PBIS implementation?

\section{Stage IV: Closure (5-10 minutes)}

Well that is all I have for you today. Before we conclude, are there any questions whatsoever? [PROVIDE TIME FOR ANY QUESTIONS]

Again, we thank you for your willingness to participate in this focus group and let your voice be heard. 
APPENDIX E: NPT PROTOCOL

DATA ANALYSIS - NORMALIZATION PROCESS THEORY

\begin{tabular}{|c|c|}
\hline Coherence & Cognitive Participation \\
\hline $\begin{array}{l}\text { What is the shared understanding of } \\
\text { the goals/expectations of PBIS? (SL, } \\
\text { DL) } \\
\text { Do CS understand their } \\
\text { roles/responsibilities of PBIS in action? } \\
\text { (SL, DL) } \\
\text { What are the shared values and beliefs } \\
\text { of PBIS? (SL, DL) }\end{array}$ & $\begin{array}{l}\text { Do CS know the key leaders who drive } \\
\text { the work of PBIS? (SL, DL) } \\
\text { - How do CS show/demonstrate "buy- } \\
\text { in"? (SL,DL) } \\
\text { - How do CS feel about their involvement } \\
\text { in PBIS implementation?(DL) } \\
\text { - How do CS define their actions in } \\
\text { sustaining PBIS? (SL) }\end{array}$ \\
\hline Collective Action & Reflexive Monitoring \\
\hline $\begin{array}{ll} & \text { How do CS interact with colleagues and } \\
\text { supervisors to implement PBIS? (SL) } \\
\text { - How do CS build their knowledge base } \\
\text { of PBIS components? (DL) } \\
\text { - How is PBIS integrated into CS daily } \\
\text { work? (SL) }\end{array}$ & $\begin{array}{l}\text { How do CS view their effectiveness } \\
\text { with implementation of PBIS? (SL) } \\
\text { How do CS evaluate PBIS practices? } \\
\text { (DL) } \\
\text { - How do CS view PBIS as it applies to } \\
\text { their capacity to work with children in } \\
\text { a variety of settings? (SL) } \\
\text { How do CS receive feedback on their } \\
\text { implementation of PBIS strategies? (SL, } \\
\text { DL) }\end{array}$ \\
\hline
\end{tabular}

SL $=$ School Leadership

DL $=$ District Leadership

CS $=$ Classified Staff 


\section{APPENDIX F: 2019 SELF-ASSESSMENT SURVEY (SELECTED FEATURES)}

\begin{tabular}{|c|c|c|c|}
\hline Feature & School & $\begin{array}{l}\text { Current In- } \\
\text { Place Status } \\
\text { Percent }\end{array}$ & $\begin{array}{l}\text { Highest } \\
\text { Improvement } \\
\text { Priority Level } \\
\text { Percent }\end{array}$ \\
\hline \multicolumn{4}{|l|}{ System: School-Wide } \\
\hline \multirow[t]{5}{*}{$\begin{array}{l}\text { Small number (e.g. 3-5) of positively and clearly stated } \\
\text { student expectations or rules are defined. }\end{array}$} & Starfield & 100 & Low 64 \\
\hline & Fairview & 63 & Med 44 \\
\hline & Kingstown & 62 & Low 48 \\
\hline & Ashford Academy & 61 & High 44 \\
\hline & Woodrow Wilson & 82 & Low 58 \\
\hline \multirow{5}{*}{ Expected student behaviors are taught directly. } & Starfield & 75 & Low $42 \%$ \\
\hline & Fairview & 53 & High 50 \\
\hline & Kingstown & 53 & High 48 \\
\hline & Ashford Academy & 52 & Med 44 \\
\hline & Woodrow Wilson & 31 & High 56 \\
\hline \multirow{5}{*}{$\begin{array}{l}\text { Problem behavior (failure to meet expected student } \\
\text { behaviors) are clearly defined. }\end{array}$} & Starfield & 57 & Low 42 \\
\hline & Fairview & 59 & High 59 \\
\hline & Kingstown & 41 & High 48 \\
\hline & Ashford Academy & 36 & Med 56 \\
\hline & Woodrow Wilson & 28 & High 56 \\
\hline \multirow[t]{5}{*}{ Consequences for problem behaviors are defined clearly. } & Starfield & 43 & High 55 \\
\hline & Fairview & 33 & High 79 \\
\hline & Kingstown & 33 & High 58 \\
\hline & Ashford Academy & 18 & High 62 \\
\hline & Woodrow Wilson & 15 & High 66 \\
\hline \multirow{5}{*}{$\begin{array}{l}\text { Options exist to allow classroom instruction to continue } \\
\text { when problem behavior occurs. }\end{array}$} & Starfield & 46 & High 50 \\
\hline & Fairview & 27 & High 74 \\
\hline & Kingstown & 33 & High 59 \\
\hline & Ashford Academy & 36 & High 44 \\
\hline & Woodrow Wilson & 33 & Med 52 \\
\hline \multirow{5}{*}{$\begin{array}{l}\text { Procedures are in place to address emergency/dangerous } \\
\text { situations. }\end{array}$} & Starfield & 83 & Low 50 \\
\hline & Fairview & 66 & High 63 \\
\hline & Kingstown & 63 & High 48 \\
\hline & Ashford Academy & 67 & High 46 \\
\hline & Woodrow Wilson & 54 & Med 46 \\
\hline \multirow{5}{*}{$\begin{array}{l}\text { A team exists for behavior support planning \& problem } \\
\text { solving. }\end{array}$} & Starfield & 77 & H/L 42 \\
\hline & Fairview & 46 & High 47 \\
\hline & Kingstown & 55 & High 38 \\
\hline & Ashford Academy & 84 & Low 52 \\
\hline & Woodrow Wilson & 44 & Med 50 \\
\hline \multirow{5}{*}{$\begin{array}{l}\text { School administrator is an active participant on the } \\
\text { behavior support team. }\end{array}$} & Starfield & 87 & Low 58 \\
\hline & Fairview & 67 & High 53 \\
\hline & Kingstown & 68 & High 42 \\
\hline & Ashford Academy & 94 & Low 59 \\
\hline & Woodrow Wilson & 56 & Med 50 \\
\hline
\end{tabular}




\begin{tabular}{|c|c|c|c|}
\hline \multirow{5}{*}{$\begin{array}{l}\text { Patterns of student problem behavior are reported to } \\
\text { teams and faculty for active decision making on a regular } \\
\text { basis (e.g. monthly). }\end{array}$} & Starfield & 61 & High 45 \\
\hline & Fairview & 41 & High 56 \\
\hline & Kingstown & 58 & H/M 36 \\
\hline & Ashford Academy & 45 & Med 40 \\
\hline & Woodrow Wilson & 22 & Med 56 \\
\hline \multirow{5}{*}{$\begin{array}{l}\text { School-wide behavior support team has a budget for (a) } \\
\text { teaching students, (b) on-going rewards, and (c) annual } \\
\text { staff planning. }\end{array}$} & Starfield & \multirow{5}{*}{$\begin{array}{l}38 \\
41 \\
21 \\
44 \\
42\end{array}$} & High 44 \\
\hline & Fairview & & H/M 36 \\
\hline & Kingstown & & Med 50 \\
\hline & Ashford Academy & & $\mathrm{M} / \mathrm{L} 43$ \\
\hline & Woodrow Wilson & & Med 58 \\
\hline \multirow{5}{*}{$\begin{array}{l}\text { All staff are involved directly and/or indirectly in school- } \\
\text { wide interventions }\end{array}$} & Starfield & \multirow{5}{*}{$\begin{array}{l}48 \\
34 \\
35 \\
45 \\
35\end{array}$} & High 42 \\
\hline & Fairview & & High 50 \\
\hline & Kingstown & & High 56 \\
\hline & Ashford Academy & & High 48 \\
\hline & Woodrow Wilson & & Med 58 \\
\hline \multirow{5}{*}{$\begin{array}{l}\text { The school team has access to on-going training and } \\
\text { support from district personnel. }\end{array}$} & Starfield & 62 & Low 63 \\
\hline & Fairview & 48 & H/M 36 \\
\hline & Kingstown & 61 & $\mathrm{H} / \mathrm{M} / \mathrm{L}$ tied $33 \%$ \\
\hline & Ashford Academy & 61 & Low 43 \\
\hline & Woodrow Wilson & 45 & Med 50 \\
\hline \multicolumn{4}{|l|}{ System: NonClassroom Setting } \\
\hline \multirow{5}{*}{$\begin{array}{l}\text { School-wide expected student behaviors apply to non- } \\
\text { classroom settings. }\end{array}$} & Starfield & 86 & High 38 \\
\hline & Fairview & 55 & High 72 \\
\hline & Kingstown & 53 & High 50 \\
\hline & Ashford Academy & 61 & Med 48 \\
\hline & Woodrow Wilson & 42 & High 42 \\
\hline \multirow{5}{*}{$\begin{array}{l}\text { School-wide expected student behaviors are taught in } \\
\text { non-classroom settings. }\end{array}$} & Starfield & 68 & H/L 38 \\
\hline & Fairview & 46 & High 76 \\
\hline & Kingstown & 50 & High 44 \\
\hline & Ashford Academy & 32 & Med 56 \\
\hline & Woodrow Wilson & 15 & Med 50 \\
\hline \multirow{5}{*}{$\begin{array}{l}\text { Staff receive regular opportunities for developing and } \\
\text { improving active supervision skills. }\end{array}$} & Starfield & 58 & High 42 \\
\hline & Fairview & 30 & High 67 \\
\hline & Kingstown & 33 & H/L 36 \\
\hline & Ashford Academy & 60 & Low 38 \\
\hline & Woodrow Wilson & 33 & Med 38 \\
\hline \multirow{5}{*}{$\begin{array}{l}\text { All staff are involved directly or indirectly in } \\
\text { management of non-classroom settings. }\end{array}$} & Starfield & 65 & Low 54 \\
\hline & Fairview & 50 & High 53 \\
\hline & Kingstown & 35 & High 50 \\
\hline & Ashford Academy & 71 & High 43 \\
\hline & Woodrow Wilson & 33 & Med 43 \\
\hline \multicolumn{4}{|l|}{ System: Classroom } \\
\hline \multirow{5}{*}{$\begin{array}{l}\text { Teachers have regular opportunities for access to } \\
\text { assistance and recommendations (observation, } \\
\text { instruction, and coaching). }\end{array}$} & Starfield & 53 & High 50 \\
\hline & Fairview & 64 & High 60 \\
\hline & Kingstown & 48 & High 40 \\
\hline & Ashford Academy & 61 & High 42 \\
\hline & Woodrow Wilson & 62 & Low 52 \\
\hline \multicolumn{4}{|l|}{ System: Individual Student } \\
\hline \multirow{5}{*}{$\begin{array}{l}\text { A behavior support team responds promptly (within } 2 \\
\text { working days) to student who present chronic problem } \\
\text { behaviors. }\end{array}$} & Starfield & 61 & High 50 \\
\hline & Fairview & 33 & High 88 \\
\hline & Kingstown & 38 & High 65 \\
\hline & Ashford Academy & 56 & High 35 \\
\hline & Woodrow Wilson & 32 & High 50 \\
\hline
\end{tabular}


Behavior is monitored and feedback provided regularly to the behavior support team and relevant staff.

Bold font indicates $\%<50$

\begin{tabular}{|l|l|l|} 
Starfield & 69 & High 55 \\
\hline Fairview & $\mathbf{4 2}$ & High 67 \\
\hline Kingstown & $\mathbf{4 0}$ & High 44 \\
\hline Ashford Academy & 55 & H/M 37 \\
\hline Woodrow Wilson & $\mathbf{4 3}$ & Med 50 \\
\hline
\end{tabular}




\section{APPENDIX G: RESEARCHER POSITIONALITY}

My background in the PBIS initiative to promote within the school district is significant. I have participated in multiple trainings and have led PBIS committees at three schools. Additionally, I participated in the district's prior behavior support initiatives between 2008 and 2012 that were precursors to the current PBIS/MTSS policies. During that time, I was one of three administrators that worked with the previous superintendent to ensure that schools were focusing on the social and emotional needs of children within a specific protocol. As a practitioner within the district under study, I had to ensure that bias regarding the district policy was addressed explicitly.

Additionally, I am aware of the concerns of teachers and school administrators regarding the implementation of PBIS within a large school district based on feedback I have received while leading PBIS teams. However, the inclusion of classified staff was rarely discussed in trainings other than a recommendation that "best practice" for implementation requires input from all stakeholders, such as classified staff (cite). I acknowledge my assumptions about the current level of involvement of classified staff in PBIS, but prior to this study, I had no evidence to confirm or contradict my assumptions. This study provided insight into the perspectives and lived experiences of the classified staff in this school district.

These experiences deserved consideration as I conducted the study to ensure that internal biases did not influence the research process. Milner (2007) warns researchers of dangers that can emerge when researchers do not consider cultural awareness, consciousness, and positionality. I addressed these concerns during the interviews and focus group questions for leading words or phrasing. Providing participants the 
opportunity to review transcripts after the interviews to verify and confirm their responses allowed me to give voice to participants. 


\section{APPENDIX H: ONLINE OPEN RESPONSE SURVEY}

I developed the following open response survey questions to engage classified staff perspectives at participating schools. The survey was distributed to all classified staff via an email link from their school administrator. The purpose of sending the survey out in addition to the focus groups was to provide any staff who chose to respond and opportunity to provide information regarding PBIS at their schools and in their personal job category even if they chose not to participate in one of the focus group session.

\section{Survey Questions}

1. Please provide the following information: a) Job title, b) Number of years in this job, and c) Grade level of your current job (elementary, middle, or high school).

2. Briefly describe your job responsibilities as they relate to interactions with students during instruction and /or behavior management.

3. Briefly describe the behavior expectations that are in place at your school. How do you feel students respond to those behavior expectations? Why?

4. Briefly describe your interactions with coworkers when seeking input regarding student discipline.

5. Have you participated in training that relates to PBIS? If so, please describe the training and any strategies that you have used.

The final question of the survey was an invitation to participate in a focus group. This was the only option that collected participants' personal information if they were interested in participating further in the study. 


\section{CURRICULUM VITAE}

Devon M. Woodlee

12204 Redspire Drive Unit 204 Louisville, KY. 40299 | 502-819-

1083 | devon.roberts@jefferson.kyschools.us

\section{Objective}

To pursue district leadership in the form of directorship or superintendence that will affect change for public education and provide advocacy for students who attend public schools.

Education

Rank I- school administration| 5/1/2001 | Western Kentucky University

- Major: Instructional Leadership

- Related coursework: School and district leadership, Interstate Licensure Exam. Kentucky Specialty

\section{Masters of Education | 6/1/1998 | Indiana Wesleyan University}

- Major: K-12 Education

- Related coursework: Change Leadership, Curriculum Design, Assessment

\section{Bachelors of Science- Elementary Education | 5/1/1995 | University of Louisville}

- Major: Elementary Education K - 5 Self Contained 


\section{DOCTOR OF EDUCATION- EDUCATIONAL LEADERSHIP | 12/12/2020 | University of Louisville}

- Major: Education Leadership

- Dissertation: Inclusion of classified staff in the implementation of positive behavior supports and interventions: A district case study

Skills \& Abilities

\section{Management}

- Olmsted Academy South Annual Girls Leadership Conference

o 2011 - 2013 Organization of this leadership conference-required call for presenters, scheduling, conference documentation, and community outreach. This annual conference brought in approximately 30 speakers annually and provided a unique opportunity for the girls (ages 11-14) to meet women from the Louisville community and learn about careers, nutrition, self-esteem, and networking

- JCAPA Leadership Conference

o 2011 Organization of this conference-required call for presenters, scheduling, conference materials, professional development credit documentation, and presenting. This conference was held for all of the assistant principals in JCPS.

\section{Leadership}

- Principals for Tomorrow - 2003

- Ignite Louisville/Leadership Louisville- Leaders Under 40 2009-2010

- President of Jefferson County Assistant Principal's Association - 2010 -2012

- Assistant Principal Leadership Development - Humana 2017

Experience

\section{Assistant Principal | Jefferson County Public Schools | 2006 - Current}

- As an Assistant Principal, my position requires a variety of responsibilities that include facilities and building safety, transportation planning, organization of school volunteers, 
school-based instructional planning, employee evaluation and critical conversations, student supervision, parent communication, professional development, logistical planning for school events, and community outreach.

\section{District Resource Teacher | Jefferson County Public Schools | 2004-2006}

- As a district resource teacher, my position required leading professional development sessions, curriculum planning, assessment evaluation, lesson demonstration, professional learning engagement, program evaluation, and teacher support in the form of mentoring.

Teacher | Jefferson County Public Schools | 1995 - 2004

- As a classroom teacher, my position required curriculum planning and development, assessment and evaluation of student performance, collaborative teaching and communication with parents, student progress monitoring, social and emotional student support, and extracurricular teaching opportunities.

\section{Guest LEcturer | Indiana University Southwest | 2007}

- Guest lecturer for graduate class to assist them in preparing for the School Leadership Licensure Exam

\section{Guest Speaker |University of louisville graduate class | 2008}

- Guest speaker for a graduate class discussing aspects and dynamics of the principalship. 